\title{
Isospectral sets for boundary value problems on the unit interval
}

\author{
JAMES RALSTON AND EUGENE TRUBOWITZ† \\ Department of Mathematics, University of California, Los Angeles, CA 90024, USA; \\ Mathematisches Institut, ETH-Zentrum, CH-8092 Zürich, Switzerland
}

Abstract. We analyse isospectral sets of potentials associated to a given 'generalized periodic' boundary condition $\left(\begin{array}{ll}a & b \\ c & d\end{array}\right)$ in $\operatorname{SL}(2, \mathbf{R})$ for the Sturm-Liouville equation on the unit interval. This is done by first studying the larger manifold $\boldsymbol{M}$ of all pairs of boundary conditions and potentials with a given spectrum and characterizing the critical points of the map from $M$ to the trace $a+d$. Isospectral sets appear as slices of $M$ whose geometry is determined by the critical point structure of the trace function. This paper completes the classification of isospectral sets for all real self-adjoint boundary conditions.

\section{Introduction}

We consider the differential operators

$$
L(q) y=-y^{\prime \prime}+q(x) y, \quad x \in[0,1],
$$

for $q$ real-valued and square-integrable, i.e. $q \in L_{\mathbf{R}}^{2}[0,1]$, with real self-adjoint boundary conditions. Considering boundary data $\left(y(0), y^{\prime}(0), y(1), y^{\prime}(1)\right)$ as vectors in $\mathbf{R}^{4}$, the set of real self-adjoint boundary conditions can be identified with the set of Lagrangian planes in $\mathbf{R}^{4}$, i.e. the set of two-dimensional subspaces on which the bilinear form $\langle v, w\rangle=v_{1} w_{2}-v_{2} w_{1}-v_{3} w_{4}+v_{4} w_{3}$ vanishes. The real self-adjoint boundary conditions split naturally into the 'separated' boundary conditions, i.e. those of the form

$$
y(0) \cos \alpha+y^{\prime}(0) \sin \alpha=0, \quad y(1) \cos \beta+y^{\prime}(1) \sin \beta=0,
$$

where $(\alpha, \beta) \in[0, \pi) \times[0, \pi)$, and the 'generalized periodic' boundary conditions, i.e. those of the form

$$
\left(\begin{array}{ll}
a & b \\
c & d
\end{array}\right)\left(\begin{array}{l}
y(1) \\
y^{\prime}(1)
\end{array}\right)=\left(\begin{array}{l}
y(0) \\
y^{\prime}(0)
\end{array}\right)
$$

where $\operatorname{det}\left(\begin{array}{ll}a & b \\ c & d\end{array}\right)=1$. The separated boundary conditions include the Dirichlet $(\alpha=\beta=0)$ and Neumann $(\alpha=\beta=\pi / 2)$ boundary conditions, and the generalized periodic boundary conditions include the periodic $\left(\left(\begin{array}{ll}a & b \\ b & d\end{array}\right)=I\right)$ and anti-periodic $\left(\left(\begin{array}{ll}a & b \\ c & d\end{array}\right)=-I\right)$ boundary conditions.

† With an appendix by Johan Tysk, Department of Mathematics, University of California, Los Angeles, CA 90024, USA. 
Our objective here is an inverse spectral theory for $L$. Given a spectrum, i.e. the eigenvalues of $L$ with their multiplicities for given boundary conditions, we would like as complete as possible a description of the set of $q \in L_{\mathbf{R}}^{2}[0,1]$ for which $L$ with the given boundary conditions has the given spectrum. This paper is intended as the last in a series that began with the study of the periodic boundary condition in [5]. The case of the Dirichlet boundary condition was discussed in some detail in [6]. The remaining separated cases were studied in [1], [3] and [4]. Finally Johan Tysk considered the generalized periodic case with $b=0$. Since his work has not appeared elsewhere, though it was completed before this paper was begun, we have included it here as Appendix A.

The case of $b \neq 0$ in the generalized periodic boundary condition is the subject of this paper. In the following sense this is the most general case. There is a natural sequence of degenerations of these boundary conditions. One can send $b \downarrow 0$, then $a \rightarrow 1$ and finally $c \rightarrow 0$, thus moving from the boundary conditions here down to the periodic case. In the other direction one can reach the separated conditions

$$
-d_{0} y(0)+y^{\prime}(0)=0, \quad a_{0} y(1)+y^{\prime}(1)=0
$$

by letting $b \uparrow \infty$ in the generalized periodic conditions

$$
\left(\begin{array}{cc}
a_{0} b & b \\
a_{0} d_{0} b-b^{-1} & d_{0} b
\end{array}\right)\left(\begin{array}{l}
y(1) \\
y^{\prime}(1)
\end{array}\right)=\left(\begin{array}{l}
y(0) \\
y^{\prime}(0)
\end{array}\right),
$$

and then send $a_{0} \rightarrow \infty$ and finally $d_{0} \rightarrow \infty$, thus moving from the boundary conditions here to the Dirichlet case. Each of the steps described above produces significant changes in the asymptotic behaviour of the eigenvalues as they tend to $\infty$ and/or in the structure of the isospectral sets. At one extreme the Dirichlet isospectral sets with the topology induced from $L_{\mathbf{R}}^{2}[0,1]$ are homeomorphic to $l^{2}(Z)$, while at the other extreme the periodic isospectral sets are compact tori, generically of infinite dimension. In this paper we will begin to see how the isospectral sets for generalized periodic boundary conditions 'interpolate' between these extremes.

As in the study of the separated boundary conditions (0.1) in [4], we have found it easier to begin by considering the set of potentials and boundary conditions which give a fixed spectrum. Identifying boundary conditions with matrices

$$
B=\left(\begin{array}{ll}
a & b \\
c & d
\end{array}\right)
$$

we let $M$ be the set of

$$
(q, B) \in L_{\mathbf{R}}^{2}[0,1] \times(\operatorname{SL}(2, \mathbf{R}) \backslash\{b=0\})
$$

such that $L(q)$ with boundary condition $B$ has the spectrum of $L\left(q_{0}\right)$ with boundary condition $B_{0}$. For all choices of $\left(q_{0}, B_{0}\right)$ in $L_{\mathbf{R}}^{2}[0,1] \times(\operatorname{SL}(2, \mathbf{R}) \backslash\{b=0\})$ the structure of $M$ is essentially the same. On $M$ one has a countable family of periodic commuting flows which fix the entry $d$ in $B$, and $d$ takes all real values on $M$. Using the flows, one can map $M$ into an infinite product of circles, and this map is injective on each level set $M \cap\{d=\delta\}, \delta \in \mathbf{R}$. However, $M \cap\{d=\delta\}$ is only a subset of the infinite product of circles. The full description of $M \cap\{d=\delta\}$ is given in $\S 3$, but one can 
picture it simply as follows: if we let $\theta_{n} \in[-n, n)$ parametrize the $n$th circle, then the image of $M \cap\{d=\delta\}$ is

$$
\left\{\left(\theta_{1}, \theta_{2}, \ldots\right): \sum\left(\theta_{n}-\theta(\delta)\right)^{2}<\infty\right\},
$$

where the constant $\theta(\delta)$ goes from $\infty$ to $-\infty$ as $\delta$ goes from $\infty$ to $-\infty$. Note that one cannot change $d$ on $M$ without changing an infinite number of the $\theta_{n}$ as well.

Once we have the explicit description of $M \cap\left\{d=d_{0}\right\}$ outlined in the preceding paragraph, it remains to fix the remaining entries in $B$ and study $M \cap\left\{B=B_{0}\right\}$. This brings us back to the problem of isospectral sets of $q$ for fixed boundary conditions. Fortunately, $b$ is constant on $M$, and hence, since $\operatorname{det} B=1$,

$$
M \cap\left\{B=B_{0}\right\}=M \cap\left\{a=a_{0}, d=d_{0}\right\},
$$

so that we only need to study the level sets of the entry $a$ as a function on $M \cap\{d=\delta\}$. To get information on the topology of these level sets, we need to study the critical points of $a$ on $M \cap\{d=\delta\}$. At this point a fortunate accident occurs. It happens that $p$ is a critical point for $a$ as a function on $M \cap\{d=\delta\}$ if and only if $p$ is a critical point for $a+d$ as a function on $M$. Moreover, $p$ is critical for $a+d$ on $M$ if and only if it is fixed by the involution

$$
q(x) \rightarrow q(1-x), \quad\left(\begin{array}{ll}
a & b \\
c & d
\end{array}\right) \rightarrow\left(\begin{array}{ll}
d & b \\
c & a
\end{array}\right) .
$$

This involution leaves $M$ invariant and is the extension of the involution for the boundary conditions $(0.1)$,

$$
q(x) \rightarrow q(1-x), \quad a_{0} \rightarrow d_{0}, \quad d_{0} \rightarrow a_{0},
$$

which was important in [4]. To each fixed point $p$ on $M$ for this involution we assign an index $I$, a finite subset of the non-negative integers. The cardinality of $I$ is the Morse index of $p$ as a critical point of $a$ on $M \cap\{d=d(p)\}$. The main result of $\S 4$ is that for each finite subset $I$ there is a unique fixed point $p_{I}$ on $M$ of index $\mathbf{I}$.

When $I$ is the empty set $\varnothing, a+d$ has a strict global maximum on $M$ at $p_{\varnothing}$, so that $M \cap\left\{B=B\left(p_{\varnothing}\right)\right\}$ is a singleton. When $a_{0}+d_{0}$ is not a critical value of $a+d$ on $M$, it turns out that the topology of $M \cap\left\{B=B_{0}\right\}$ is determined by $a_{0}+d_{0}$ alone. If $a_{0}+d_{0}$ is not a critical value, then all the homotopy groups of $M \cap\left\{B=B_{0}\right\}$ are isomorphic to those of a rather explicit subset of $M$ whose topology only depends on $\left\{I: a_{0}+d_{0}<a\left(p_{I}\right)+d\left(p_{1}\right)\right\}$; cf. theorem 6.4. The main point at which our analysis is incomplete is that we have not shown that the only accumulation point for critical values of $a+d$ on $M$ is $-\infty$. If that were established, then, as $a_{0}+d_{0}$ went from $a\left(p_{\varnothing}\right)+d\left(p_{\varnothing}\right)$ to $-\infty$, one would see the progression in discrete steps of the homotopy of $M \cap\left\{B=B_{0}\right\}$ from triviality to the full homotopy of $M$.

As in the case of periodic boundary conditions, Floquet theory plays a fundamental role in the spectral theory here. In this paragraph we will outline the Floquet theory for generalized periodic boundary conditions (with $b \neq 0$ ). In doing this we will also fix some notation. We let $y_{i}(x, \lambda, q), i=1,2$, be the solutions of

$$
-y^{\prime \prime}+q y=\lambda y
$$


for $\lambda \in \mathbf{C}$ such that the matrix

$$
F(x, \lambda, q)=\left(\begin{array}{ll}
y_{1} & y_{2} \\
y_{1}^{\prime} & y_{2}^{\prime}
\end{array}\right)
$$

satisfies $F(0, \lambda, q)=I$. Letting $B$ denote the boundary condition matrix $\left(\begin{array}{ll}a & b \\ c & d\end{array}\right)$ as before, we define the 'discriminant'

$$
\Delta(\lambda, q)=\operatorname{tr}(B F(1, \lambda, q))
$$

and check that $\lambda$ is an eigenvalue for the boundary condition $B$ if and only if $\Delta(\lambda)=2$. Moreover, $\Delta(\lambda)$ is an entire function of $\lambda$ of order $\frac{1}{2}$ (cf. theorem 1.1), and for the boundary condition given by $B$ with $b \neq 0$ one can recover $b$ and hence the top-order asymptotic of $\Delta(\lambda)$ as $\lambda \rightarrow \infty$ from the spectrum (cf. theorems 1.1 and 1.4). Hence by Hadamard's theorem, given the spectrum of one of the problems considered here-without specifying which one- $\Delta(\lambda)$ is determined. In particular the roots of $\Delta(\lambda)=-2$, i.e. the spectrum with $B$ replaced by $-B$, are determined. For this reason one can without loss of generality assume that both spectra are given and restrict oneself to the case $b>0$, as we will from here on. As in the periodic case, $\Delta(\lambda) \rightarrow \infty$ on the real axis as $\lambda \rightarrow-\infty$ (assuming $b>0$ ), and, as $\lambda \rightarrow \infty$, $\Delta(\lambda)$ has an infinite sequence of non-degenerate minima less than or equal to -2 followed by non-degenerate maxima greater than or equal to 2 and no other real critical values (cf. Appendix B). Hence the roots of $\Delta(\lambda)=2$ and $\Delta(\lambda)=-2$ listed by multiplicity interlace in pairs once one is above $\lambda_{0}$, the first root of $\Delta(\lambda)=2$; i.e.

$$
\lambda_{0}<\lambda_{1} \leq \lambda_{2}<\lambda_{3} \leq \lambda_{4}<\cdots,
$$

where $\Delta\left(\lambda_{4 k-1}\right)=\Delta\left(\lambda_{4 k}\right)=2$ and $\Delta\left(\lambda_{4 k+1}\right)=\Delta\left(\lambda_{4 k+2}\right)=-2$. We will often refer to the sequence $\left\{\lambda_{k}\right\}_{k=0}^{\infty}$ as the 'generalized periodic spectrum', even though strictly speaking it is the union of two spectra. These spectra are listed by multiplicity as eigenvalues, since $\lambda_{2 k}=\lambda_{2 k-1}$ if and only if $\lambda_{2 k}$ is an eigenvalue of multiplicity two (cf. Appendix B).

The spectrum of $-y^{\prime \prime}+q y$ with the boundary conditions $y(0)=0$ and $a y(1)+$ $b y^{\prime}(1)=0$ is given by the roots of $\left[a y_{2}+b y_{2}^{\prime}\right](1, \lambda)=0$. Since this boundary value problem is self-adjoint, all these roots are real, and they are also simple (cf. (1.2)). Hence we list them as a strictly increasing sequence $\left\{\mu_{j}\right\}_{j+1}^{\infty}$. We call $\left\{\mu_{j}\right\}_{j=1}^{\infty}$ the generalized Dirchlet spectrum, since it reduces to the Dirichlet spectrum when $b=0$. The generalized periodic and generalized Dirichlet spectra are related by the identity (cf. lemma 4.2(iv))

$$
\left[y_{2}^{2}+b \Delta(\lambda) y_{2}+b^{2}\right](1, \lambda)=\left[\left(a y_{2}+b y_{2}^{\prime}\right)\left(d y_{2}+b y_{1}\right)\right](1, \lambda)
$$

Since $y_{2}(1, \lambda)$ is real for $\lambda$ real, this implies that the $\mu_{j}$ lie in the 'gaps', i.e. in the set where $|\Delta(\lambda)| \geq 2$. In the case of periodic boundary conditions there is exactly one Dirichlet eigenvalue in each gap above $\lambda_{0}$. Since $\Delta(\lambda, q, B)$ and the $\mu_{j}$, being simple roots, depend continuously on $B$, it follows that for $b \neq 0$ there is exactly one generalized Dirichlet eigenvalue in each gap above $\lambda_{0}$, i.e. $\lambda_{2 j-1} \leq \mu_{j} \leq \lambda_{2 j}$ for $j \geq 1$.

The plan of this long paper is fairly straightforward. In $\S 1$ we determine the asymptotic behaviour of the sequences $\left\{\lambda_{k}\right\}$ and $\left\{\mu_{k}\right\}$ as $k \rightarrow \infty$ and show that the 
generalized Dirichlet spectra together with $b, d$ and the 'norming constants' $\kappa_{k}=y_{2}\left(1, \mu_{k}, q\right)$ form an analytic coordinate system on

$$
L_{\mathbf{R}}^{2}[0,1] \times(\operatorname{SL}(2, \mathbf{R}) \backslash\{b=0\}) .
$$

The point here is not only that these data determine $(q, B)$ uniquely, but also that, with the proper Hilbert manifold structure on the space $S$ of sequences

$$
\left(b, d, \mu_{1}, \kappa_{1}, \mu_{2}, \kappa_{2}, \ldots\right) \text {, }
$$

the Jacobian of the coordinate mapping is boundedly invertible so that the inverse mapping is also analytic. This section is closely related to the early chapters of [6].

In $\$ 2$ we present flows generated by analytic vector fields $V_{k}$ on $L_{\mathbf{R}}^{2}[0,1] \times$ (SL $(2, \mathbf{R}) \backslash\{b=0\})$ which preserve $M$ and move $\left(\mu_{k}, \kappa_{k}\right)$ around the (topological) circle $\kappa_{k}^{2}+b \Delta\left(\mu_{k}\right) \kappa_{k}+b^{2}=0$ while fixing $b, d$ and $\left(\mu_{j}, \kappa_{j}\right), j \neq k$. As in [4] and [6], these flows can be explicitly integrated to give formulae for $q$ and $B$ on the orbits. The formula for $q$ does not play a role in the sections that follow, but we use the formula for $B$ frequently.

In $\S 3$ we identify $M$ with an explicit analytic submanifold $N$ of $S$ by using the flows of $\S 2$ to show that the range of the coordinate functions is dense in $N$, from which it follows that the range is equal to $N$ since $M$ is closed and the inverse coordinate map is analytic. $M$ thus inherits the analytic structure of $N$.

After some preliminary lemmas, $\S 4$ is devoted to the proof of the existence of a unique critical point of $a+d$ on $M$ with index $\mathbf{I}$. For the proof of uniqueness we use the explicit integration of the flows and the involution. The flows, however, do not seem to be useful in proving the existence of critical points. For our proof of existence we use another fortunate accident: for $k$ sufficiently large the minimum of

$$
\int_{0}^{1}|k+q(x)|^{2} d x
$$

on a properly chosen subset of $M$ is assumed at $p_{l}$. For example,

$$
\min _{M \cap\{a=d\}} \int_{0}^{1}|k+q(x)|^{2} d x
$$

is assumed at $p_{\varnothing}$ for $k$ sufficiently large.

In $\S 5$ we show that the range of $B$ on $M$ is

$$
B\left(p_{\varnothing}\right) \cup\left\{\left(\begin{array}{ll}
a & b \\
c & d
\end{array}\right): b=b_{0}, \operatorname{det} B=1, a+d<a\left(p_{\varnothing}\right)+d\left(p_{\varnothing}\right)\right\}
$$

and prove generalizations of this which will be used in $\S 6$. Formula (0.2) shows that $d$ takes all real values on $M$, which had not been proven earlier in the paper.

In the final section, $\S 6$, we collect all the information which we have obtained on the isospectral sets $M \cap\left\{B=B_{0}\right\}$. We show that they are always connected, are non-compact when they are not singletons, and are analytic submanifolds of $L_{\mathbf{R}}^{2}[0,1]$ unless they contain fixed points of the involution. We conclude with the result on the homotopy groups of $M \cap\left\{B=B_{0}\right\}$ described earlier.

For the boundary conditions considered by Tysk in Appendix A there is a full set of isospectral flows fixing the boundary conditions, so that the description of 
the isospectral sets for fixed boundary conditions become analogous to the description of $M \cap\{d=\delta\}$. This makes some of the analysis nearly identical to $\S \S 1,2$ and 3 and we have not presented it in detail.

Finally we should point that we have not solved the problem considered in [2], [3] and [6] of determining what sequences $\left\{\lambda_{k}\right\}$ arise. It would be interesting to learn if anything like the result of [2] for the periodic spectrum is valid here.

\section{A coordinate system}

This section is devoted to the construction of a global real analytic coordinate system on $L_{\mathbf{R}}^{2}[0,1] \times(\operatorname{SL}(2, \mathbf{R}) \backslash\{b=0\})$. We will also determine the asymptotic behaviour of the generalized periodic eigenvalues. The development follows chapters 2 and 3 of Pöschel and Trubowitz [6] closely, and we state two basic theorems from [6] here for future reference.

THEOREM 1.1 (theorem 1.3 of $[6])$. On $[0,1] \times C \times L_{\mathrm{C}}^{2}[0,1]$

(i) $\left|y_{1}(x, \lambda, q)-\cos \lambda^{1 / 2} x\right| \leq \frac{1}{\left|\lambda^{1 / 2}\right|} \exp \left(\left|\operatorname{Im} \lambda^{1 / 2}\right| x+\|q\| x^{1 / 2}\right)$,

(ii) $\left|y_{2}(x, \lambda, q)=\frac{\sin \lambda^{1 / 2} x}{\lambda^{1 / 2}}\right| \leq \frac{1}{|\lambda|} \exp \left(\left|\operatorname{Im} \lambda^{1 / 2}\right| x+\|q\| x^{1 / 2}\right)$,

(iii) $\left|y_{1}^{\prime}(x, \lambda, q)+\lambda^{1 / 2} \sin \lambda^{1 / 2} x\right| \leq\|q\| \exp \left(\left|\operatorname{Im} \lambda^{1 / 2}\right| x+\|q\| x^{1 / 2}\right)$,

(iv) $\left|y_{2}^{\prime}(x, \lambda, q)-\cos \lambda^{1 / 2} x\right| \leq \frac{\|q\|}{\left|\lambda^{1 / 2}\right|} \exp \left(\left|\operatorname{Im} \lambda^{1 / 2}\right| x+\|q\| x^{1 / 2}\right)$.

By the Riesz representation theorem the Frechet derivative $d_{q} F(v)$ of a function on $L^{2}$ can be written $d_{q} F(v)=\int_{0}^{1}(\partial F / \partial q(t)) v(t) d t$. The following theorem gives the 'gradient' $\partial F / \partial q(t)$ for the $y$.

Theorem 1.2 (theorem 1.6 of [6]). For $j=1,2$

$$
\begin{aligned}
& \frac{\partial y_{j}}{\partial q(t)}(x)=y_{j}(t)\left(y_{1}(t) y_{2}(x)-y_{1}(x) y_{2}(t)\right) \chi_{[0, x]}(t), \\
& \frac{\partial y_{j}^{\prime}}{\partial q(t)}(x)=y_{j}(t)\left(y_{1}(t) y_{2}^{\prime}(x)-y_{1}^{\prime}(x) y_{2}(t)\right) \chi_{[0, x]}(t),
\end{aligned}
$$

where $\chi_{E}(t)$ is the characteristic function of $E$. These gradients are continuous in $(x, \lambda, q)$. In addition

$$
\frac{\partial y_{j}}{\partial \lambda}=-\int_{0}^{1} \frac{\partial y_{j}}{\partial q(t)} d t \quad \text { and } \quad \frac{\partial y_{j}^{\prime}}{\partial \lambda}=-\int_{0}^{1} \frac{\partial y_{j}^{\prime}}{\partial q(t)} d t .
$$

We refer the reader to [6] for proofs of theorems 1.1 and 1.2. Theorem 1.2 is an easy computation. Theorem 1.1 is also quite standard in various forms. We need the particular form given here to control $y_{1}$ and $y_{2}$ for complex $\lambda$ and for $q$ in bounded sets of $L^{2}$.

We begin by studying the generalized Dirichlet spectrum. 
THEOREM 1.3. The generalized Dirichlet eigenvalues $\mu_{n}(q, a, b), n=1,2, \ldots$, are real analytic functions on $L_{\mathbf{R}}^{2}[0,1] \times\left(\mathbf{R}^{2} \backslash 0\right)$. When $b \neq 0$ they satisfy the asymptotic estimate

$$
\mu_{n}(q, a, b)=\left(n-\frac{1}{2}\right)^{2} \pi^{2}+2 a / b+\int_{0}^{1} q d x+r_{n},
$$

where $\left\{r_{n}\right\} \in I^{2}(Z)$. For convenience here and elsewhere we will denote this by

$$
\mu_{n}(q, a, b)=\left(n-\frac{1}{2}\right)^{2} \pi^{2}+2 a / b+\int_{0}^{1} q d x+l^{2}(n) .
$$

Remark. When $a \neq 0$ and $b=0$, the set of $\mu_{n}$ becomes the Dirichlet spectrum and

$$
\mu_{n}(q, a, 0)=n^{2} \pi^{2}+\int_{0}^{1} q d x+l^{2}(n) .
$$

Thus the asymptotics change drastically when $b=0$. However, (1.1) does hold uniformly in the sense that $\left\|\left\{r_{n}\right\}\right\|_{2}$ is uniformly bounded on sets where $\|q\|_{2}$ and $|a| /|b|$ are bounded.

Proof. This is precisely the analogue of parts of theorems 2.3 and 2.4 of [6]. We will only sketch the proof, indicating the modifications needed in the arguments of chapter 2 of [6].

Multiplying the identity

$$
\left(-\frac{d^{2}}{d x^{2}}+q-\mu\right) \frac{\partial y_{2}}{\partial \mu}=y_{2}
$$

by $y_{2}$ and integrating from 0 to 1 in $x$, one has

$$
\int_{0}^{1} y_{2}^{2}(x, \mu) d x=\left(-y_{2} \frac{\partial y_{2}^{\prime}}{\partial \mu}+y_{2}^{\prime} \frac{\partial y_{2}}{\partial \mu}\right)(1, \mu) .
$$

Evaluating at $\mu=\mu_{n}$ gives

$$
\int_{0}^{1} y_{2}^{2}(x, \mu) d x= \begin{cases}-\frac{y_{2}\left(1, \mu_{n}\right)}{b}\left(a \frac{\partial y_{2}}{\partial \mu}+b \frac{\partial y_{2}^{\prime}}{\partial \mu}\right)\left(1, \mu_{n}\right), & b \neq 0 \\ \frac{y_{2}^{\prime}\left(1, \mu_{n}\right)}{a}\left(a \frac{\partial y_{2}}{\partial \mu}+b \frac{\partial y_{2}^{\prime}}{\partial \mu}\right)\left(1, \mu_{n}\right), & a \neq 0 .\end{cases}
$$

Thus, since $a \partial y_{2} / \partial \mu\left(1, \mu_{n}, q\right)+b \partial y_{2}^{\prime} / \partial \mu\left(1, \mu_{n}, q\right) \neq 0$, the analyticity of $\mu_{n}(q, a, b)$ follows from the implicit function theorem applied to the equation $a y_{2}\left(1, \mu_{n}, q\right)+$ $b_{2}^{\prime}\left(1, \mu_{n}, q\right)=0$.

The estimates of theorem 1.1 show that for $\lambda \in \mathbf{C}$

$$
\left|\frac{a}{b} y_{2}(1, \lambda, q)+y_{2}^{\prime}(1, \lambda, q)-\cos \lambda^{1 / 2}\right| \leq \frac{1}{|b|}\left[\frac{|a|}{|\lambda|^{1 / 2}}+\left(\frac{|a|}{|\lambda|}+\frac{|b|\|q\|}{|\lambda|^{1 / 2}}\right) e^{\|q\|}\right] e^{\mid l m \lambda^{1 / 2}} \text {. }
$$

Thus, as in lemma 2.2 of [6], Rouché's theorem can be used to show that, for $N>N(|a| /|b|,\|q\|), a y_{2}(1, \lambda, q)+b y_{2}^{\prime}(1, \lambda, q)$ has exactly $N$ roots in $\operatorname{Re} \lambda<N^{2} \pi^{2}$ and exactly one root in $\left|\lambda^{1 / 2}-\left(N-\frac{1}{2}\right) \pi\right|<\pi / 2$. This gives

$$
\left(\mu_{n}\right)^{1 / 2}=\left(n-\frac{1}{2}\right) \pi+O(1)
$$


To improve (1.3) to (1.1), one can use the identity

$$
\mu_{n}-\left(n-\frac{1}{2}\right)^{2} \pi^{2}=\int_{0}^{1} \frac{d}{d t} \mu_{n}(t q, t a, b) d t
$$

together with

$$
\begin{aligned}
\frac{\partial \mu_{n}}{\partial q(x)} & =\frac{y_{2}^{2}\left(x, \mu_{n}\right)}{\left\|y_{2}\left(\cdot, \mu_{n}\right)\right\|^{2}}=2 \sin ^{2}\left(\mu_{n}\right)^{1 / 2} x+O\left(\frac{1}{n}\right) \\
& =1-\cos 2\left(\mu_{n}\right)^{1 / 2} x+O(1 / n)
\end{aligned}
$$

and

$$
\frac{\partial \mu_{n}}{\partial a}=\frac{y_{2}^{2}\left(1, \mu_{n}\right)}{b\left\|y_{2}\left(\cdot, \mu_{n}\right)\right\|^{2}} .
$$

This gives $\mu_{n}=\left(n-\frac{1}{2}\right)^{2} \pi^{2}+O(1)$ and $\left(\mu_{n}\right)^{1 / 2}=\left(n-\frac{1}{2}\right) \pi+O(1 / n)$, so that

$$
\cos 2\left(\mu_{n}\right)^{1 / 2} x=\cos (2 n-1) \pi x+O(1 / n) .
$$

Using this improvement when one again substitutes (1.5) into (1.4), one derives (1.1).

The same methods will be used to find the asymptotics of the generalized periodic spectrum.

THEOREM 1.4. The generalized periodic eigenvalues $\lambda_{n}(q, B)$ satisfy the asymptotic estimates when $b \neq 0$ :

$$
\begin{aligned}
& \lambda_{2 n}(q, B)=n^{2} \pi^{2}+\frac{2}{b}(a+d-2)+\int_{0}^{1} q d x+l^{2}(n), \\
& \lambda_{2 n+1}(q, B)=n^{2} \pi^{2}+\frac{2}{b}(a+d+2)+\int_{0}^{1} q d x+l^{2}(n) .
\end{aligned}
$$

Hence $b$ and $2(a+d)+b \int_{0}^{1} q d x$ are spectral invariants.

Proof. The eigenvalues $\lambda_{n}(q, B)$ are the roots of $\Delta^{2}(\lambda, q, B)-4=0$. Using

$$
\Delta(\lambda)=a y_{1}(1, \lambda)+b y_{1}^{\prime}(1, \lambda)+c y_{2}(1, \lambda)+d y_{2}^{\prime}(1, \lambda)
$$

and the estimates of theorem 1.1, we have for $|\lambda|>1$

$$
\left|\Delta^{2}(\lambda)-4-b^{2} \lambda \sin ^{2} \lambda^{1 / 2}\right| \leq C(\|q\|, B)|\lambda|^{1 / 2} e^{2 \mid \operatorname{Im} \lambda^{1 / 2}} \text {. }
$$

Thus, using Rouché's theorem on the boundary of

$$
\left\{|\operatorname{Re} z| \leq\left(n+\frac{1}{4}\right)^{2} \pi^{2}\right\} \cap\{|\operatorname{Im} z| \leq K\},
$$

one sees that, for $n \geq N(\|q\|,\|B\|, 1 /|b|), \Delta^{2}(\lambda)-4$ has exactly $2 n+2$ roots in the half-plane $\operatorname{Re} \lambda<\left(n+\frac{1}{4}\right)^{2} \pi^{2}$. On the other hand, Rouché's theorem applied on the contour $\left|\lambda^{1 / 2}-n \pi\right|=\pi / 4$ shows $\Delta^{2}(\lambda)-4$ has exactly two roots in the region $\mid \lambda^{1 / 2}-$ $n \pi \mid<\pi / 4$. These conclusions do not require det $B=1$ and hold uniformly on sets of $(q, B)$ where $\|q\|,\|B\|$ and $1 /|b|$ are bounded. In the case that det $B \neq 1$ all the roots of $\Delta^{2}(\lambda)-4=0$ need not be real, but again, since $\Delta^{2}(\lambda)-4$ is always real-valued for $\lambda$ real, (1.7) implies that, for $n>N(\|q\|, 1 /|b|,\|B\|), \Delta^{2}(\lambda)-4$ always has at least two real roots in the open interval $\left(\pi^{2}\left(n-\frac{1}{4}\right)^{2}, \pi^{2}\left(n+\frac{1}{4}\right)^{2}\right)$. Hence, combining this with the argument from Rouchés theorem, we conclude that, for $n \geq$ 
$N(\|q\|, 1 /|b|,\|B\|), \Delta^{2}(\lambda)-4$ has exactly two roots in $\left|\lambda^{1 / 2}-n \pi\right|<\pi / 4$ and they are real, and exactly $2 n+2$ roots in the half-plane $\operatorname{Re}\{\lambda\}<\left(n+\frac{1}{4}\right)^{2} \pi^{2}$.

Combining theorems 1.1 and 1.2, one computes for real $\lambda$

$$
\frac{\partial \Delta(\lambda)}{\partial \lambda}=-\frac{b \cos \lambda^{1 / 2}}{2}+O\left(\frac{1}{\lambda^{1 / 2}}\right)
$$

where again this holds uniformly on sets where $\|q\|, 1 /|b|$ and $\|B\|$ are bounded. Thus for $n \geq N(\|q\|, 1 /|b|,\|B\|)$ the two roots of $\Delta^{2}(\lambda)-4$ in $\left|\lambda^{1 / 2}-n \pi\right|<\pi / 4$ are smooth functions of $(q, B)$ by the implicit function theorem, and we label them $\lambda_{2 n}(q, B)<\lambda_{2 n+1}(q, B)$.

Now we are in a position to finish the proof of theorem 1.4 in the same way as theorem 1.3. Since we require only $b \neq 0$, we have for $k=2 n, 2 n+1$, $n \geq N(\|q\|, 1 /|b|,\|B\|)$

$$
\lambda_{k}(q, B)-\lambda_{k}^{0}=\int_{0}^{1} \frac{d}{d t} \lambda_{k}(t q, t a, b, t c, t d) d t .
$$

The $\lambda_{k}^{0}$ are the roots of

$$
b^{2} \lambda^{2} \sin ^{2} \lambda^{1 / 2}=4 \quad \text { in }\left(\pi^{2}\left(n-\frac{1}{4}\right)^{2}, \pi^{2}\left(n+\frac{1}{4}\right)^{2}\right)
$$

and hence

$$
\lambda_{2 n}^{0}=n^{2} \pi^{2}-\frac{4}{b}+O\left(\frac{1}{n}\right), \quad \lambda_{2 n+1}^{0}=n^{2} \pi^{2}+\frac{4}{b}+O\left(\frac{1}{n}\right) .
$$

Again combining theorems 1.1 and 1.2, one computes for real $\lambda$

$$
\frac{\partial \Delta(\lambda)}{\partial q(x)}=b\left(\cos \lambda^{1 / 2} \frac{1+\cos 2 \lambda^{1 / 2} x}{2}+\sin \lambda^{1 / 2} \frac{\sin 2 \lambda^{1 / 2} x}{2}\right)+O\left(\frac{1}{\lambda^{1 / 2}}\right) .
$$

Hence, using (1.8), implicit differentiation and $\left|\left(\lambda_{k}\right)^{1 / 2}-n \pi\right|<\pi / 4$, one computes for $k=2 n, 2 n+1$

$$
\begin{aligned}
\frac{\partial \lambda_{k}}{\partial q(x)} & =\frac{1}{2}+\frac{\cos 2\left(\lambda_{k}\right)^{1 / 2} x}{2}+\tan \left(\lambda_{k}\right)^{1 / 2} \frac{\sin 2\left(\lambda_{k}\right)^{1 / 2} x}{2}+O\left(\frac{1}{n}\right), \\
\frac{\partial \lambda_{k}}{\partial a} & =\frac{2}{b}+O\left(\frac{1}{n}\right), \quad \frac{\partial \lambda_{k}}{\partial c}=O\left(\frac{1}{n}\right), \quad \frac{\partial \lambda_{k}}{\partial d}=\frac{2}{b}+O\left(\frac{1}{n}\right),
\end{aligned}
$$

uniformly for $n \geq N(\|q\|, 1 /|b|,\|B\|)$. Using (1.10) in (1.9), one sees

$$
\lambda_{k}=n^{2} \pi^{2}+O(1)
$$

and hence $\left(\lambda_{k}\right)^{1 / 2}=n \pi+O(1 / n)$. Thus $(1.10)$ shows

$$
\frac{\partial \lambda_{k}}{\partial q(x)}=\frac{1}{2}+\frac{\cos 2 \pi n x}{2}+O\left(\frac{1}{n}\right)
$$

Substituting this together with the rest of (1.10) into (1.9) gives (1.6).

We now give the analogue for the generalized Dirichlet spectrum of the well known result (theorem 3.5 in [6]) that the spectrum plus the 'norming constants' determine the potential. In the generalized Dirichlet case these data determine the ratio $a / b$ as well, but the method of proof is the same. 
TheOREM 1.5. If $\mu_{n}(q, a, b)=\mu_{n}(\tilde{q}, \tilde{a}, \tilde{b}) \equiv \mu_{n}, n \geq 1$, and $y_{2}\left(1, \mu_{n}, q\right)=y_{2}\left(1, \mu_{n}, \tilde{q}\right) \equiv$ $y_{2}\left(1, \mu_{n}\right), n \geq 1$, then $q=\tilde{q}$ and $a / b=\tilde{a} / \tilde{b}$.

Proof. Following the method of theorems 3.3 and 3.5 in [6], we introduce $z(x, \lambda, q, c)$, the solution of

$$
-z^{\prime \prime}+q z=\lambda z
$$

satisfying $z(1, \lambda, q, c)=-1, z^{\prime}(1, \lambda, q, c)=c$. Then for $n \geq 1$

$$
\begin{aligned}
& y_{2}\left(x, \mu_{n}, q\right)=-y_{2}\left(1, \mu_{n}\right) z\left(x, \mu_{n}, q, a / b\right), \\
& y_{2}\left(x, \mu_{n}, \tilde{q}\right)=-y_{2}\left(1, \mu_{n}\right) z\left(x, \mu_{n}, \tilde{q}, \tilde{a} / \tilde{b}\right) .
\end{aligned}
$$

Consider the function

$$
f(\lambda)=\frac{\left(y_{2}(x, \lambda, q)-y_{2}(x, \lambda, \tilde{q})\right)(z(x, \lambda, q, a / b)-z(x, \lambda, \tilde{q}, \tilde{a} / \tilde{b}))}{a y_{2}(1, \lambda, q)+b y_{2}^{\prime}(1, \lambda, q)}
$$

Note that the numerator is entire and the denominator is entire with simple zeros at the $\mu_{n}$ (see theorem 1.3) and no other zeros. Thus $f(\lambda)$ is meromorphic with simple poles at $\lambda=\mu_{n}, n \geq 1$, with residues

$$
R_{n}=\frac{-y_{2}\left(1, \mu_{n}, q\right)}{a\left(\partial y_{2} / \partial \mu\right)\left(1, \mu_{n}, q\right)+b\left(\partial y_{2}^{\prime} / \partial \mu\right)\left(1, \mu_{n} q\right)}\left(z\left(x, \mu_{n}, q, a / b\right)-z\left(x, \mu_{n}, \tilde{q}, \tilde{a} / \tilde{b}\right)\right)^{2}
$$

Since (1.2) implies

$$
R_{n}=\frac{y_{2}^{2}\left(1, \mu_{n}, q\right)}{b \int_{0}^{1} y_{2}^{2}\left(x, \mu_{n}, q\right) d x}\left(z\left(x, \mu_{n}, q, a / b\right)-z\left(x, \mu_{n}, \tilde{q}, \tilde{a} / \tilde{b}\right)\right)^{2}
$$

we see $R_{n} \geq 0$.

The estimates of theorem 1.1 show that

$$
|f(\lambda)| \leq C \frac{e^{\left|\operatorname{Im} \lambda^{1 / 2}\right| x}}{|\lambda|} \frac{e^{\mid \operatorname{Im} \lambda^{1 / 2 \mid(1-x)}}}{|\lambda|^{1 / 2}} /\left[b \cos \lambda^{1 / 2}+O\left(\frac{e^{\left|\operatorname{Im} \lambda^{1 / 2}\right|}}{|\lambda|^{1 / 2}}\right)\right]
$$

Here we used

$$
z(x, \lambda, q, c)=-y_{1}\left(1-x, q^{*}\right)+c y_{2}\left(1-x, q^{*}\right),
$$

where $q^{*}(x)=q(1-x)$, in estimating $z(x, q, a / b)-z(x, \tilde{q}, \tilde{a} / \tilde{b})$. Thus, taking $r_{n}=\left(n-\frac{1}{2}\right)^{2} \pi^{2}$, we see $\lim _{n \rightarrow \infty} \operatorname{Max}_{|\lambda|=r_{n}}|\lambda f(\lambda)|=0$. Thus $R_{n}=0$ for all $n, f$ is entire, and we see $f(\lambda) \equiv 0$ for $x \in[0,1]$. In particular

$$
y_{2}\left(x, \mu_{1}, q\right)=y_{2}\left(x, \mu_{1}, \tilde{q}\right) \text {, }
$$

so that a.e. in $[0,1]$

$$
0=(q(x)-\tilde{q}(x)) y_{2}\left(x, \mu_{1}, q\right) .
$$

Since $y_{2}$ has only a finite number of zeros, we conclude

$$
q=\tilde{q} .
$$

Hence $\int_{0}^{1} q d x=\int_{0}^{1} \tilde{q} d x$ and (1.1) implies $a / b=\tilde{a} / \tilde{b}$.

If $(q, B)$ is an element of $L^{2}[0,1] \times(\operatorname{SL}(2, \mathbf{R}) \backslash\{b=0\})$, theorem 1.5 implies $(q, B)$ is uniquely determined by

$$
\left\{\mu_{n}(q, a, b)\right\}_{n=1}^{\infty}, \quad\left\{y_{2}\left(1, \mu_{n}(q, a, b), q\right)\right\}_{n=1}^{\infty}, \quad b \quad \text { and } \quad d .
$$


As earlier, we use the notation $B=\left(\begin{array}{cc}a & b \\ c & d\end{array}\right)$. Thus these data could be used as coordinates on $L^{2}[0,1] \times(\operatorname{SL}(2, \mathbf{R}) \backslash\{b=0\})$. However, to construct analytic coordinates from them, we will need several preliminary results. We begin with the analogue of theorem 1.3 for the norming constants.

THEOREM 1.6. The norming constants $y_{2}\left(1, \mu_{n}(q, a, b), q\right), n=1,2, \ldots$, are analytic functions on $L^{2}[0,1] \times\left(\mathbf{R}^{2} \backslash 0\right)$. When $b \neq 0$ they satisfy the asymptotic estimate

$$
y_{2}\left(1, \mu_{n}(q, a, b), q\right)=\frac{(-1)^{n+1}}{\left(n-\frac{1}{2}\right) \pi}+\frac{1}{n^{2}} l^{2}(n) .
$$

Proof. Since $y_{2}(1, \lambda, q)$ is analytic in $(\lambda, q)$ and $\mu_{n}(q, a, b)$ is analytic by theorem 1.3 , the analyticity is immediate.

For the asymptotics we use the analogue of (1.4):

$$
y_{2}\left(1, \mu_{n}\left(q, a_{1} b_{1}\right), q\right)-\frac{(-1)^{n+1}}{\left(n-\frac{1}{2}\right) \pi}=\int_{0}^{1} \frac{d}{d t}\left(y_{2}\left(1, \mu_{n}(t q, t a, b), t q\right)\right) d t
$$

Using theorems 1.1 and 1.2 , we have

$$
\begin{aligned}
\frac{\partial y_{2}(1, \lambda, q)}{\partial q(x)} & =y_{2}(x, \lambda)\left(y_{2}(1, \lambda) y_{1}(x, \lambda)-y_{1}(1, \lambda) y_{2}(x, \lambda)\right) \\
& =\frac{-\cos \lambda^{1 / 2}}{2 \lambda}+\frac{\cos \lambda^{1 / 2}(2 x-1)}{2 \lambda}+O\left(\lambda^{-3 / 2}\right), \\
\frac{\partial y_{2}}{\partial y} & =-\int_{0}^{1} \frac{\partial y_{2}}{\partial q(x)} d x .
\end{aligned}
$$

Combining (1.12) with the estimates on $\mu_{n}$, and $\partial \mu_{n} / \partial q(x)$ and $\partial \mu_{n} / \partial a$ from theorem 1.3 and its proof, gives (1.11).

Theorems 1.3 and 1.6 show that the mapping $\Phi:(q, B)$

$$
\rightarrow\left(b, d, \mu_{1}(q, a, B), y_{2}\left(1, \mu_{1}(q, a, b), q\right), \mu_{2}(q, a, b), y_{2}\left(1, \mu_{2}(q, a, b), q\right), \ldots\right)
$$

takes $L_{\mathbf{R}}^{2}(0,1) \times \operatorname{SL}(2, \mathbf{R}) \backslash\{b=0\}$ into $S$, the space of real sequences

$$
s=\left(b, d, \mu_{1}, \kappa_{1}, \mu_{2}, \kappa_{2}, \ldots\right),
$$

where

$$
\mu_{n}=\left(n-\frac{1}{2}\right)^{2} \pi^{2}+r+l^{2}(n)
$$

and

$$
\kappa_{n}=\frac{(-1)^{n+1}}{\left(n-\frac{1}{2}\right) \pi}+\frac{1}{n^{2}} l^{2}(n) .
$$

To make $S$ into a (trivial) real analytic manifold modelled on $l_{R}^{2}$, we introduce the global coordinates on $S$, assigning

$$
\tilde{s}=\left(b, d, r, \tilde{\mu}_{1}, \tilde{\kappa}_{1}, \tilde{\mu}_{2}, \tilde{\kappa}_{2}, \ldots\right)
$$


to $s$, where

$$
\tilde{\mu}_{n}=\mu_{n}\left(n-\frac{1}{2}\right)^{2} \pi^{2}-r
$$

and

$$
\tilde{\kappa}_{n}=2 \pi^{2} n^{2}\left(\kappa_{n}+\frac{(-1)^{n}}{\left(n-\frac{1}{2}\right) \pi}\right) .
$$

We claim that $\Phi$ is a real analytic mapping of $L_{\mathbf{R}}^{2}[0,1] \times(\operatorname{SL}(2, \mathbf{R}) \backslash\{b=0\})$ into $S$. For this we need to show that given $\left(q_{0}, B_{0}\right) \in L_{\mathbf{R}}^{2}[0,1] \times(\operatorname{SL}(2, \mathbf{R}) \backslash\{b=0\})$, the functions $\mu_{n}(q, a, b)$ and $y_{2}\left(1, \mu_{n}(q, a, b), q\right)$ are analytic on a (complex) neighbourhood $U$ of $\left(q_{0}, B_{0}\right)$ in $L^{2}[0,1] \times M_{22}$, independent of $n$, and that $\tilde{\Phi}(U)$ is a bounded set in $l^{2}$ (see theorem A.3 of [6]). The proofs of these facts follow the proofs of the corresponding results for the Dirichlet spectrum in theorems 3.1 and 3.6 in [6] so closely that we will omit them here.

As we observed earlier, theorem 1.5 implies that $\Phi$ is globally one-to-one. The final step in showing that $\Phi$ is a global coordinate function is showing its derivative $\Phi^{\prime}$ is boundedly invertible at all points of $L_{\mathbf{R}}^{2}[0,1] \times(\operatorname{SL}(2, \mathbf{R}) \backslash\{b=0\})$.

THEOREM 1.7. In terms of the coordinates on $S, \Phi^{\prime}(q, B)$ is the linear mapping

$$
(\dot{q}, \dot{B}) \rightarrow\left(\dot{b}, \dot{d}, 2\left(\frac{\dot{a}}{b}-\frac{a \dot{b}}{b^{2}}\right)+\int_{0}^{1} \dot{q} d x,\left\langle\tilde{\mu}_{1}^{\prime}(q, a, b),(\dot{q}, \dot{B})\right\rangle,\left\langle\tilde{\kappa}_{1}^{\prime}(q, a, b),(\dot{q}, \dot{B})\right\rangle, \ldots\right),
$$

where $\dot{q} \in L^{2}[0,1], \dot{B}=\left(\begin{array}{c}\dot{a} \dot{b} \\ \dot{c} \dot{d}\end{array}\right)$ with $\dot{c}=(\dot{a} d+\dot{d} a) / b+\dot{b}(1-a d) / b^{2}$, i.e. $\dot{B}$ is in the tangent space to $\operatorname{SL}(2, \mathbf{R})$ at $B$. The pairings $\left\langle\tilde{\mu}_{n}^{\prime}(q, a, b),(\dot{q}, \dot{B})\right\rangle$ and $\left\langle\tilde{\kappa}_{n}^{\prime}(q, a, b)\right.$, $(\dot{q}, \dot{B})\rangle$ are given by

$$
\begin{aligned}
\left\langle\tilde{\mu}_{n}^{\prime}(q, a, b),(\dot{q}, \dot{B})\right\rangle= & -2\left(\frac{\dot{a}}{b}-\frac{a \dot{b}}{b^{2}}\right)-\int_{0}^{1} \dot{q} d x \\
& +\left\|y_{2}\left(\mu_{n}\right)\right\|_{2}^{-2}\left[\int_{0}^{1} y_{2}^{2}\left(\mu_{n}\right) \dot{q} d x+\left(\frac{\dot{a}}{b}-\frac{a b}{b^{2}}\right) y_{2}^{2}\left(1, \mu_{n}\right)\right]
\end{aligned}
$$

and

$$
\begin{aligned}
\left(2 \pi^{2} n^{2}\right)^{-1}\left\langle\tilde{\kappa}_{n}^{\prime}(q, a, b),(\dot{q}, \dot{B})\right\rangle & \\
= & y_{2}\left(1, \mu_{n}\right) \int_{0}^{1} y_{1}\left(\mu_{n}\right) y_{2}\left(\mu_{n}\right) \dot{q} d x-y_{1}\left(1, \mu_{n}\right) \int_{0}^{1} y_{2}^{2}\left(\mu_{n}\right) \dot{q} d x \\
& -\left(y_{2}\left(1, \mu_{n}\right) \int_{0}^{1} y_{1}\left(\mu_{n}\right) y_{2}\left(\mu_{n}\right) d x-y_{1}\left(1, \mu_{n}\right) \int_{0}^{1} y_{2}^{2}\left(\mu_{n}\right) d x\right) \\
& \times\left[\left\langle\tilde{\mu}_{n}^{\prime}(q, a, b),(\dot{q}, \dot{B})\right\rangle+2\left(\frac{\dot{a}}{b}-\frac{a \dot{b}}{b^{2}}\right)+\int_{0}^{1} \dot{q} d x\right] .
\end{aligned}
$$

The mapping $\Phi^{\prime}$ is boundedly invertible.

Proof. The formulae for $\left\langle\tilde{\mu}_{n}^{\prime},(\dot{q}, \dot{B})\right\rangle$ and $\left\langle\tilde{\kappa}_{n}^{\prime},(\dot{q}, \dot{B})\right\rangle$ are computed using $(1.5)$ and (1.12). 
Once again using the asymptotics for the $\mu_{n}$ in $n$, and $y_{1}$ and $y_{2}$ in $\lambda$, one has

$$
\begin{aligned}
w_{n} \equiv & \left\|y_{2}\left(\mu_{n}\right)\right\|_{n}^{-2} y_{2}^{2}\left(\mu_{n}\right)-1=-\cos (2 n-1) \pi x+O(1 / n), \\
g_{n}= & (1 / b)\left(\left\|y_{2}\left(\mu_{n}\right)\right\|_{2}^{-2} y_{2}^{2}\left(1, \mu_{n}\right)-2\right)=O(1 / n), \\
z_{n}= & 2 \pi^{2} n^{2}\left(y_{2}\left(1, \mu_{n}\right) y_{1}\left(\mu_{n}\right) y_{2}\left(\mu_{n}\right)-y_{1}\left(1, \mu_{n}\right) y_{2}^{2}\left(\mu_{n}\right)\right) \\
& -2 \pi^{2} n^{2}\left(\int_{0}^{1}\left(y_{2}\left(1, \mu_{n}\right) y_{1}\left(\mu_{n}\right) y_{2}\left(\mu_{n}\right)-y_{1}\left(1, \mu_{n}\right) y_{2}^{2}\left(\mu_{n}\right)\right) d x\right) \\
& \times\left\|y_{2}\left(\mu_{n}\right)\right\|_{2}^{-2} y_{2}^{2}\left(\mu_{n}\right) \\
= & \sin (2 n-1) \pi x+O(1 / n), \\
h_{n}= & -\frac{2 \pi^{2} n^{2}}{b}\left(\int_{0}^{1}\left(y_{2}\left(1, \mu_{n}\right) y_{1}\left(\mu_{n}\right) y_{2}\left(\mu_{n}\right)-y_{1}\left(1, \mu_{n}\right) y_{2}^{2}\left(\mu_{n}\right)\right) d x\right) \\
& \times\left\|y_{2}\left(\mu_{n}\right)\right\|_{2}^{-2} y_{2}^{2}\left(1, \mu_{n}\right) \\
= & O(1 / n) .
\end{aligned}
$$

Hence

$$
\begin{aligned}
\Phi^{\prime}(q, B)[\dot{q}, \dot{B}]= & \dot{b} f_{1}+\dot{d} f_{2} \\
& +\left(0,0, \frac{2 \dot{a}}{b}+\int_{0}^{1} \dot{q} d x, \int_{0}^{1} w_{1} \dot{q} d x-\dot{a} g_{1}, \int_{0}^{1} z_{1} \dot{q} d x+\dot{a} h_{1}, \ldots\right),
\end{aligned}
$$

where $f_{i} \in l^{2}, i=1,2$, and

$$
f_{1}=(1,0, \ldots), \quad f_{2}=(0,1,0, \ldots) .
$$

Thus to show that $\Phi^{\prime}(q, B)$ is boundedly invertible, it will suffice to show that

$$
\psi:(\dot{q}, \dot{a}) \rightarrow\left(\frac{2 \dot{a}}{b}+\int_{0}^{1} \dot{q} d x, \int_{0}^{1} w_{1} \dot{q} d x+\dot{a} g_{1}, \int_{0}^{1} z_{1} \dot{q} d x+\dot{a} h_{1}, \ldots\right)
$$

is boundedly invertible. Moreover, (1.14) implies

$$
\infty>\sum_{n=1}^{\infty}\left(\left\|\left\{w_{n}, g_{n}\right\}-\{-\cos (2 n-1) \pi, 0\}\right\|^{2}+\left\|\left\{z_{n}, h_{n}\right\}-\{\sin (2 n-1) \pi, 0\}\right\|^{2}\right),
$$

where for $\{w, g\} \in L^{2}[0,1] \times \mathbf{C}$

$$
\|\{w, g\}\|^{2}=\int_{0}^{1}|w|^{2} d x+|g|^{2} .
$$

Hence, applying theorem D.3 of [6] to the complete orthonormal sequence $\{0,1\}$, $\left\{2^{1 / 2} \cos (2 n-1) \pi x, 0\right\},\left\{2^{1 / 2} \sin (2 n-1) \pi x, 0\right\}, n=1, \ldots$, in $L^{2}[0,1] \times \mathbf{C}$, we see that to show $\psi$ is boundedly invertible it will suffice to show that $\left\{\{1,2 / b\},\left\{w_{n}, g_{n}\right\}\right.$, $\left.\left\{z_{n}, g_{n}\right\},\left\{z_{n}, h_{n}\right\}, n=1,2, \ldots\right\}$ is linearly independent in the sense that no element is in the closed linear span of the others.

The key to establishing linear independence is the following observation which we will use again in $\S 2$. If we let $w_{+}$and $w_{-}$denote two solutions of $-u^{\prime \prime}+q u=\lambda u$ with $\lambda=\mu$, and $z_{+}$and $z_{-}$denote a second pair with $\lambda=\nu, \mu \neq \nu$, then, letting 


$$
\begin{aligned}
& {[f, g]=f g^{\prime}-g f^{\prime}, } \\
& 2 \int_{0}^{1} w_{+} w_{-}\left(z_{+} z_{-}\right)^{\prime} d x=\left.\left(w_{+} w_{-} z_{+} z_{-}\right)\right|_{0} ^{1}+\int_{0}^{1}\left(w_{+} w_{-}\left(z_{+} z_{-}\right)^{\prime}-\left(w_{+} w_{-}\right)^{\prime} z_{+} z_{-}\right) d x \\
&=\left.\left(w_{+} w_{-} z_{+} z_{-}\right)\right|_{0} ^{1}+\int_{0}^{1}\left(w_{+} z_{+}\left[w_{-}, z_{-}\right]+w_{-} z_{-}\left[w_{+}, z_{+}\right]\right) d x \\
&=\left.\left(w_{+} w_{-} z_{+} z_{-}\right)\right|_{0} ^{1}+\frac{1}{\mu-\nu} \int_{0}^{1}\left(\left[w_{+}, z_{+}\right]\left[w_{-}, z_{-}\right]\right)^{\prime} d x \\
&=\left.\left(w_{+} w_{-} z_{+} z_{-}\right)\right|_{0} ^{1}+\left.\frac{1}{\mu-\nu}\left[w_{+}, z_{+}\right]\left[w_{-}, z_{-}\right]\right|_{0} ^{1} .
\end{aligned}
$$

Using (1.15) with $w_{+} w_{-}=y_{2}^{2}\left(\mu_{n}\right)$ and $z_{+} z_{-}=y_{2}^{2}\left(\mu_{m}\right)$, we have

$$
0=\int_{0}^{1} w_{n}\left(y_{2}^{2}\left(\mu_{n}\right)\right)^{\prime} d x-g_{n} \frac{b}{2} y_{2}^{2}\left(1, \mu_{m}\right)
$$

for all $m=1,2, \ldots$ Similarly for $m \neq n$

$$
0=\int_{0}^{1} z_{n}\left(y_{2}^{2}\left(\mu_{m}\right)\right)^{\prime} d x-h_{n} \frac{b}{2} y_{2}^{2}\left(1, \mu_{n}\right),
$$

and trivially

$$
0=\int_{0}^{1}\left(y_{2}^{2}\left(\mu_{m}\right)\right)^{\prime} d x-\frac{2}{b} \frac{b}{2} y_{2}^{2}\left(1, \mu_{n}\right) .
$$

However,

$$
\begin{aligned}
& \int_{0}^{1} z_{n}\left(y_{2}^{2}\left(\mu_{n}\right)\right)^{\prime} d x-h_{n} \frac{b}{2} y_{2}^{2}\left(1, \mu_{n}\right) \\
& \quad=\pi^{2} n^{2} \int_{0}^{1}\left(y_{2}\left(\mu_{n}\right) y_{2}\left(1, \mu_{n}\right) y_{1}\left(\mu_{n}\right)-y_{1}\left(1, \mu_{n}\right) y_{2}\left(\mu_{n}\right)\left(y_{2}\left(\mu_{n}\right)^{2}\right)^{\prime}\right) d x \\
& =\pi^{2} n^{2} \int_{0}^{1} y_{2}^{2}\left(\mu_{1}\right) y_{2}\left(1, \mu_{n}\right) d x \neq 0 .
\end{aligned}
$$

Formulae (1.16)-(1.19) show that no $\left\{z_{n}, h_{n}\right\}$ is in the closed linear span of the other vectors.

By the same method

$$
\begin{aligned}
& 0=\int_{0}^{1} z_{n}\left(z_{m}\right)^{\prime} d x-\frac{b^{2}}{4} h_{n} h_{m}, \\
& 0=\int_{0}^{1}\left(z_{m}\right)^{\prime} d x-\frac{2}{b} \frac{b^{2}}{4} h_{m} \quad \text { for all } m
\end{aligned}
$$

and for $m \neq n$

$$
0=\int_{0}^{1} w_{n}\left(z_{m}\right)^{\prime} d x-\frac{b^{2}}{4} g_{n} h_{m} .
$$

However, by (1.19)

$$
\int_{0}^{1} w_{n}\left(z_{n}\right)^{\prime} d x-\frac{b^{2}}{4} g_{n} h_{n} d x \neq 0
$$


Thus we may conclude that no $\left\{w_{n}, g_{n}\right\}$ is in the span of the other vectors.

Finally we note that

$$
\int_{0}^{1} z_{n} d x=\int_{0}^{1} w_{n} d x=0
$$

so that $\{1,0\}$ is orthogonal to the span of

$$
\left\{\left\{w_{n}, g_{n}\right\},\left\{z_{n}, h_{n}\right\}, n>1\right\}
$$

but obviously not orthogonal to $\{1,2 / b\}$.

2. Flows

In this section we begin by finding vector fields $(\dot{q}, \dot{B})$ which are tangent to $M$. In the case of periodic boundary conditions, the vector field $(\dot{q}, \dot{B})$ with

$$
\dot{q}=2\left(\frac{\partial \Delta(\lambda)}{\partial q(x)}\right)^{\prime}
$$

and $\dot{B}=0$ is tangent (cf. [5]). We will use (2.1) for $\dot{q}$ here, but to get a vector field tangent to $M$ we will need to make $\dot{B}$ non-zero. One natural way to arrive at the correct expression for $\dot{B}$ is simply to compute

$$
\int_{0}^{1} \frac{\partial \Delta(\mu)}{\partial q(t)} \dot{q}(t, \nu) d t
$$

using (2.1) and then choose $\dot{B}$ so that $\dot{\Delta}(\mu)=0$. This is the approach we will take here.

From theorem 1.2 we have

$$
\begin{aligned}
\frac{\partial \Delta(\lambda)}{\partial q(x)}= & a y_{1}(x)\left(y_{2}(1) y_{1}(x)-y_{1}(1) y_{2}(x)\right)+b y_{1}(x)\left(y_{2}^{\prime}(1) y_{1}(x)-y_{1}^{\prime}(1) y_{2}(x)\right) \\
& +c y_{2}(x)\left(y_{2}(1) y_{1}(x)-y_{1}(1) y_{2}(x)\right)+d y_{2}(x)\left(y_{2}^{\prime}(1) y_{1}(x)-y_{1}^{\prime}(1) y_{2}(x)\right),
\end{aligned}
$$

where all functions are evaluated at $(\cdot, \lambda, q)$. The derivative $(\partial \Delta(\lambda) / \partial q(x))^{\prime}$ is the derivative of the right-hand side of (2.3) with respect to $x$. The key to evaluating (2.2) is the result of the computation (1.15), i.e. if we let $w_{+}$and $w_{-}$denote two solutions of $-w^{\prime \prime}+q w=\lambda w$, then

$$
2 \int_{0}^{1} w_{+} w_{-}\left(z_{+} z_{-}\right)^{\prime} d x=\left.\left(w_{+} w_{-} z_{+} z_{-}\right)\right|_{0} ^{1}+\left.\frac{1}{\mu-\nu}\left[w_{+}, z_{+}\right]\left[w_{-}, z_{-}\right]\right|_{0} ^{1} .
$$

The integrand in (2.2) is clearly a linear combination of terms like the one evaluated in (2.4). Hence one could apply (2.4) directly to (2.2). However, it is better to try first to find solutions of $-u^{\prime \prime}+q u=\lambda u, f_{+}$and $f_{-}$, such that

$$
\frac{\partial \Delta(\lambda)}{\partial q(x)}=h(\lambda) f_{+}(x, \lambda) f_{-}(x, \lambda) \text {. }
$$

Then one can evaluate (2.2) with a single application of (2.4).

The functions $f_{ \pm}$in the factorization turn out to be the solutions of $-u^{\prime \prime}+q u=\lambda u$ associated with the eigenvectors of $B F(\lambda)$. Let $f_{ \pm}(x, \lambda)=y_{1}(x)+c_{ \pm}(\lambda) y_{2}(x)$, where

$$
B F(\lambda)\left(\begin{array}{c}
1 \\
c_{ \pm}
\end{array}\right)=\xi_{ \pm}(\lambda)\left(\begin{array}{c}
1 \\
c_{ \pm}
\end{array}\right) \text {. }
$$


One computes easily

$$
f_{ \pm}=y_{1}(x, \lambda)+\frac{\xi_{ \pm}(\lambda)-a y_{1}(1, \lambda)-b y_{1}^{\prime}(1, \lambda)}{a y_{2}(1, \lambda)+b y_{2}^{\prime}(1, \lambda)} y_{2}(x, \lambda) .
$$

The eigenvalues $\xi_{ \pm}$are the roots of $\xi^{2}-\Delta(\lambda) \xi+1=0$, and hence $\xi_{+} \xi_{-}=1$ and $\xi_{+}+\xi_{-}=\Delta$. The product

$$
\begin{aligned}
f_{+} f_{-}= & y_{1}^{2}+y_{1} y_{2}\left(\frac{c y_{2}(1, \lambda)+d y_{2}^{\prime}(1, \lambda)-a y_{1}(1, \lambda)-b y_{1}^{\prime}(1, \lambda)}{a y_{2}(1, \lambda)+b y_{2}^{\prime}(1, \lambda)}\right) \\
& +y_{2}^{2}\left(\frac{1-\left(a y_{1}(1, \lambda)+b y_{1}^{\prime}(1, \lambda)\right) \Delta+\left(a y_{1}(1, \lambda)+b y_{1}^{\prime}(1, \lambda)\right)^{2}}{\left(a y_{2}(1, \lambda)+b y_{2}^{\prime}(1, \lambda)\right)^{2}}\right)
\end{aligned}
$$

To simplify this, note that the denominator of the coefficient of $\left(y_{2}(x)\right)^{2}$ equals

$$
\begin{aligned}
1-\left(a y_{1}(1, \lambda)+b y_{1}^{\prime}(1, \lambda)\right)\left(c y_{2}(1, \lambda)+d y_{2}^{\prime}(1, \lambda)\right) & \\
= & 1-a c y_{1}(1, \lambda) y_{2}(1, \lambda)-b d y_{1}^{\prime}(1, \lambda) y_{2}^{\prime}(1, \lambda)-a d\left(1+y_{2}(1, \lambda) y_{1}^{\prime}(1, \lambda)\right) \\
& \quad-b c\left(y_{1}(1, \lambda) y_{2}^{\prime}(1, \lambda)-1\right) \\
= & -\left(a y_{2}(1, \lambda)+b y_{2}^{\prime}(1, \lambda)\right)\left(c y_{1}(1, \lambda)+d y_{1}^{\prime}(1, \lambda)\right)
\end{aligned}
$$

since $a d-b c=1$. Thus

$$
\begin{aligned}
\left(a y_{2}(1, \lambda)+b y_{2}^{\prime}(1, \lambda)\right) f_{+} f_{-}= & \left(a y_{2}(1, \lambda)+b y_{2}^{\prime}(1, \lambda)\right) y_{1}^{2} \\
& +\left(c y_{2}(1, \lambda)+d y_{2}^{\prime}(1, \lambda)-a y_{1}(1, \lambda)-b y_{1}^{\prime}(1, \lambda)\right) y_{1} y_{2} \\
& +\left(-c y_{1}(1, \lambda)-d y_{2}^{\prime}(1, \lambda)\right) y_{2}^{2}=\partial \Delta(\lambda) / \partial q(x)
\end{aligned}
$$

directly from (2.3).

Now combining (2.1)-(2.5), we have

$$
\begin{aligned}
& \int_{0}^{1} \frac{\partial \Delta(\mu)}{\partial q(x)} \dot{q}(x, \nu) d x \\
& =\left.\frac{\partial \Delta(\mu)}{\partial q(x)} \frac{\partial \Delta(\nu)}{\partial q(x)}\right|_{0} ^{1} \\
& \quad+\left.\frac{\left(a y_{2}(1, \mu)+b y_{2}^{\prime}(1, \mu)\right)\left(a y_{2}(1, \nu)+b y_{2}^{\prime}(1, \nu)\right)}{\mu-\nu}\left[f_{+}(\mu), f_{+}(\nu)\right]\left[f_{-}(\mu), f_{-}(\nu)\right]\right|_{0} ^{1} .
\end{aligned}
$$

However, since $B$ is symplectic, by the definition of $f_{ \pm}$

$$
\left.\left[f_{ \pm}(\mu), f_{ \pm}(\nu)\right]\right|_{x=1}=\left.\xi_{ \pm}(\mu) \xi_{ \pm}(\nu)\left[f_{ \pm}(\mu), f_{ \pm}(\nu)\right]\right|_{x=0} .
$$

Thus

$$
0=\left.\left[f_{+}(\mu), f_{+}(\nu)\right]\left[f_{-}(\mu), f_{-}(\nu)\right]\right|_{0} ^{1}
$$

and one is left with

$$
\int_{0}^{1} \frac{\partial \Delta(\mu)}{\partial q(t)} \dot{q}(t, \nu) d t=\left.\frac{\partial \Delta(\mu)}{\partial q(t)} \frac{\partial \Delta(\nu)}{\partial q(t)}\right|_{0} ^{1} .
$$

Note that (2.6) is also true, trivially, when $\mu=\nu$.

Now we are ready to choose $\dot{B}(\lambda)$ so that the flow determined by $(\dot{q}(t, \lambda), \dot{B}(t, \lambda))$ with $\dot{q}$ given by $(2.1)$ will be isospectral. Since

$$
\frac{\partial \Delta(\lambda)}{\partial q(0)}=a y_{2}(1, \lambda)+b y_{2}^{\prime}(1, \lambda), \quad \frac{\partial \Delta(\lambda)}{\partial q(1)}=d y_{2}(1, \lambda)+b y_{1}(1, \lambda),
$$


one can use the trivial relations

$$
\begin{array}{ll}
\frac{\partial \Delta(\lambda)}{\partial a}=y_{1}(1, \lambda), & \frac{\partial \Delta(\lambda)}{\partial b}=y_{1}^{\prime}(1, \lambda), \\
\frac{\partial \Delta(\lambda)}{\partial c}=y_{2}(1, \lambda), & \frac{\partial \Delta(\lambda)}{\partial d}=y_{2}^{\prime}(1, \lambda)
\end{array}
$$

to conclude from (2.6) that the correct choice is

$$
\dot{B}=\left(\begin{array}{cc}
-b\left(d y_{2}(1, \lambda)+b y_{1}(1, \lambda)\right) & u \\
-d\left(d y_{2}(1, \lambda)+b y_{1}(1, \lambda)\right)+a\left(a y_{2}(1, \lambda)+b y_{2}^{\prime}(1, \lambda)\right) & b\left(a y_{2}(1, \lambda)+b y_{2}^{\prime}(1, \lambda)\right)
\end{array}\right) .
$$

Thus we have proven:

THEOREM 2.1. For all real $\lambda$ the vector fields $(\dot{q}, \dot{B})=V(q, B, \lambda)$, given by $(2.1)$ and (2.7), are tangent to the isospectral manifold $M$ at $(q, B)$.

Replacing the constant $\lambda$ in $V(q, B, \lambda)$ by any smooth function $\lambda(q, B)$ gives a new vector field tangent to $M$. If we let $\lambda=\mu_{n}(q, B)$, the $n$th generalized Dirichlet eigenvalue, we get an analytic vector field $V_{n}(q, B)$. The vector fields $V_{n}$ give rise to commuting isospectral flows. The proof of this will be based on the uniqueness theorem (theorem 1.6) of $\S 1$. We will show that $\mu_{m}, m \neq n$, and $y_{2}\left(1, \mu_{m}, q\right), m \neq n$, do not change under the flow of $V_{n}$ and that the motion of $\mu_{n}$ and $y_{2}\left(1, \mu_{n}, q\right)$ is determined by $\Delta(\lambda, q, B)$ alone. Since the flow is isospectral for the generalized periodic spectrum, $\Delta(\lambda, q, B)$ is constant on the orbits. Thus it will be clear that the induced flows on $\left\{\mu_{n}\right\}_{n=1}^{\infty}$ and $\left\{y_{2}\left(1, \mu_{n}, q\right)\right\}_{n=1}^{\infty}$ commute, and, since all the flows fix $b, d$ and $a d-b c$, the uniqueness theorem implies the flows commute.

We begin by showing that $\mu_{m}, m \neq n$, is constant on integral curves of $V_{n}$. Let denote the derivative along $V_{n}$. The derivative $\dot{y}_{2}(x, \mu, q)$, with $\mu$ constant, satisfies

$$
-\dot{y}_{2}^{\prime \prime}+q \dot{y}_{2}-\mu \dot{y}_{2}=-\dot{q} y_{2} \text {. }
$$

Thus

$$
\int_{0}^{1} \dot{q} y_{2}^{2}(x, \mu) d x=\left.\left(y_{2} \dot{y}_{2}^{\prime}-y_{2}^{\prime} \dot{y}_{2}\right)\right|_{x=0} ^{1}
$$

and, evaluating at $\mu=\mu_{m}$, we have

$$
\int_{0}^{1} \dot{q} y_{2}^{2}\left(x, \mu_{m}\right) d x=\frac{y_{2}\left(1, \mu_{m}\right)}{b}\left(a \dot{y}_{2}\left(1, \mu_{m}\right)+b \dot{y}_{2}^{\prime}\left(1, \mu_{m}\right)\right) \text {. }
$$

Hence, since $a(t) y_{2}\left(1, \mu_{m}(t), q(t)\right)+b y_{2}^{\prime}\left(1, \mu_{m}(t), q(t)\right)=0$ by definition, using the chain rule and substituting (1.2) and (2.8), we have

$$
0=\dot{a} y_{2}\left(1, \mu_{m}\right)+\left(\frac{-b}{y_{2}\left(1, \mu_{m}\right)} \int_{0}^{1} y_{2}^{2}\left(x, \mu_{m}\right) d x\right) \dot{\mu}_{m}+\frac{b}{y_{2}\left(1, \mu_{m}\right)} \int_{0}^{1} \dot{q} y_{2}^{2}\left(x, \mu_{m}\right) d x
$$

Thus, using (2.7),

$$
\left(\int_{0}^{1} y_{2}^{2}\left(x, \mu_{m}\right) d x\right) \dot{\mu}_{m}=\int_{0}^{1} \dot{q} y_{2}^{2}\left(x, \mu_{m}\right) d x-y_{2}^{2}\left(1, \mu_{m}\right)\left(d y_{2}\left(1, \mu_{n}\right)+b y_{1}\left(1, \mu_{n}\right)\right) \text {. }
$$


To avoid complications in the factorization (2.5) when $\lambda=\mu_{n}$, we first compute $\int_{0}^{1} \dot{q} y_{2}^{2}\left(x, \mu_{m}\right) d x$, assuming $\dot{q}$ is given by (2.1), and then pass to the limit $\lambda=\mu_{n}(q, B)$ at the end of the computation. From (2.4) and (2.5) we have

$$
\begin{aligned}
\int_{0}^{1} \dot{q} y_{2}^{2}\left(x, \mu_{m}\right) d x= & y_{2}^{2}\left(1, \mu_{m}\right) \frac{\partial \Delta(\lambda)}{\partial q(1)}+\frac{a y_{2}(1, \lambda)+b y_{2}^{\prime}(1, \lambda)}{\lambda-\mu_{m}} \\
& \times\left.\left[y_{2}\left(x, \mu_{m}\right), f_{+}(x, \lambda)\right]\left[y_{2}\left(x, \mu_{m}\right), f_{-}(x, \lambda)\right]\right|_{0} ^{1} .
\end{aligned}
$$

Note by the formula for $f_{ \pm}$

$$
\left[y_{2}\left(x, \mu_{m}\right), f_{ \pm}(x, \lambda)\right]=-1 \quad \text { at } x=0,
$$

and at $x=1$

$$
\begin{aligned}
{\left[y_{2}\left(x, \mu_{m}\right), f_{ \pm}(x, \lambda)\right] } & =\frac{y_{2}\left(1, \mu_{m}\right)}{b}\left(a f_{ \pm}(1, \lambda)+b f_{ \pm}^{\prime}(1, \lambda)\right) \\
& =\frac{y_{2}\left(1, \mu_{m}\right)}{b}\left(\frac{a\left(y_{2}(1, \lambda) \xi_{ \pm}(\lambda)+b\right)+b\left(y_{2}^{\prime}(1, \lambda) \xi_{ \pm}-a\right)}{a y_{2}(1, \lambda)+b y_{2}^{\prime}(1, \lambda)}\right) \\
& =\frac{y_{2}\left(1, \mu_{m}\right)}{b} \xi_{ \pm}(\lambda) .
\end{aligned}
$$

Moreover, by (2.3)

$$
\frac{\partial \Delta(\lambda)}{\partial q(1)}=d y_{2}(1, \lambda)+b y_{1}(1, \lambda)
$$

Thus, combining (2.9) and (2.10), we conclude that along $V_{n}$

$$
\begin{aligned}
& \left(\int_{0}^{1} y_{1}^{2}\left(x, \mu_{m}\right) d x\right) \mu_{m}=\left(\lim _{\lambda \rightarrow \mu_{m}} \frac{a y_{2}(1, \lambda)+b y_{2}^{\prime}(1, \lambda)}{\lambda-\mu_{m}}\right)\left(\frac{y_{2}^{2}\left(1, \mu_{m}\right)}{b^{2}}-1\right) \\
& \quad=\left\{\begin{array}{l}
0, \quad n \neq m, \\
{\left[a \frac{\partial y_{2}}{\partial \mu}\left(1, \mu_{m}\right)+b\left(\frac{\partial y_{2}^{\prime}}{\partial \mu}\right)\left(1, \mu_{m}\right)\right]\left(\frac{y_{2}^{2}\left(1, \mu_{m}\right)}{b^{2}}-1\right), \quad n=m .}
\end{array}\right.
\end{aligned}
$$

To further simplify the case $n=m$, one may use (2.8). The final result is: the derivative of $\mu_{m}$ along $V_{n}$ is given by

$$
\dot{\mu}_{m}=\left\{\begin{array}{l}
0 \quad \text { if } m \neq n, \\
\frac{b}{y_{2}\left(1, \mu_{n}\right)}-\frac{y_{2}\left(1, \mu_{n}\right)}{b} \quad \text { if } m=n .
\end{array}\right.
$$

Since

$$
\begin{aligned}
\Delta\left(\mu_{m}\right) & =\left.\left[a y_{1}+b\left(\frac{y_{1} y_{2}^{\prime}-1}{y_{2}}\right)+c y_{2}+d y_{2}^{\prime}\right]\right|_{\left(1, \mu_{m}\right)} \\
& =\frac{-b}{y_{2}\left(1, \mu_{m}\right)}-\frac{y_{2}\left(1, \mu_{m}\right)}{b}
\end{aligned}
$$


one sees

$$
\dot{\mu}_{n}=\sqrt{\Delta^{2}\left(\mu_{n}\right)-4}
$$

where $\sqrt{ }$ denotes either the positive or negative square root. The correct choice of $\sqrt{ }$ at $t=0$ is given in (2.11). Subsequently the sign of $\mu_{n}$ changes from + to - when $\mu_{n}$ hits $\lambda_{2 n}$, and from - to + when $\mu_{n}$ hits $\lambda_{2 n-1}$. Thus, since $\Delta(\lambda)$ is constant on integral curves of $V_{n}, \mu_{n}(t)$ is determined by the initial values of $\mu_{n}, y_{2}\left(1, \mu_{n}, q\right)$ and $\Delta(\lambda)$.

Finally, since for $\mu_{m}=\mu_{m}(q, B)$

we have

$$
\Delta\left(\mu_{m}, q, B\right)=-\frac{b}{y_{2}\left(1, \mu_{m}, q\right)}-\frac{y_{2}\left(1, \mu_{m}, q\right)}{b},
$$

$$
y_{2}\left(1, \mu_{m}, q\right)=-b / \xi\left(\mu_{m}\right),
$$

where $\xi\left(\mu_{m}\right)$ is one of the roots of $\xi^{2}-\Delta\left(\mu_{m}\right)+1$. For $m \neq n$, since $b, \Delta(\lambda)$ and $\mu_{m}$ are constant on the integral curves of $V_{n}$, and $y_{2}\left(1, \mu_{m}, q(t)\right)$ is continuous in $t$, we conclude $y_{2}\left(1, \mu_{m}, q\right)$ is constant on the integral curves too. For $m=n$ we still have $\Delta(\lambda)$ determined by the initial data and

$$
y_{2}\left(1, \mu_{n}(t), q(t)\right)=-b / \xi_{*}\left(\mu_{n}(t)\right)
$$

but now $*= \pm$ is determined by

$$
\dot{\mu}_{n}=-\xi_{*}\left(\mu_{n}(t)\right)+\xi_{*}^{-1}\left(\mu_{n}(t)\right) \text {. }
$$

Thus we have proven:

THEOREM 2.2. The flows generated by the vector fields $V_{n}, n=1, \ldots$, commute. The data $\mu_{m}$ and $y_{2}\left(1, \mu_{m}, q\right)$ are constant on the integral curves of $V_{n}$ for $m \neq n$. On -integral curves of $V_{n}, \mu_{n}$ and $y_{2}\left(1, \mu_{n}, q\right)$ are determined by (2.13), (2.14) and (2.15).

As in the earlier papers in this series, the vector fields $V_{n}$ can be integrated explicitly. As before, the integration is based on the following tricky but directly verifiable observation which actually goes back to Darboux (see the discussion in chapter 5 of [6]): if

$$
-d^{2} f / d x^{2}+q f=\alpha f
$$

and

$$
-d^{2} g / d x^{2}+q g=\beta g
$$

and we set

$$
\tilde{q}=q-2 \frac{d^{2}}{d x^{2}} \log |f|
$$

$\tilde{f}=1 / f$ and $\tilde{g}=(1 / f)[f, g]$, then

$$
-d^{2} \tilde{f} / d x^{2}+\tilde{q} \tilde{f}=\alpha \tilde{f}, \quad-d \tilde{g} / d x^{2}+\tilde{q} \tilde{\mathbf{g}}=\beta \tilde{g} .
$$

These equations only hold where $f \neq 0$, and since $f$ in general will have zeros, the usefulness of this observation is not immediately clear. However, one can repeat the argument with $\tilde{q}$ in place of $q$ and $(1 / f)[f, g]$ in place of $f$ in (2.16). Then setting

$$
\hat{q}=\tilde{q}-2 \frac{d^{2}}{d x^{2}} \log \left|\frac{1}{f}[f, g]\right|=q-2 \frac{d^{2}}{d x^{2}} \log |[f, g]|, \quad \hat{f}=\frac{f}{[f, g]},
$$


one again has $-\hat{f}^{\prime \prime}(x)+\hat{q}(x) \hat{f}(x)=\beta \hat{f}(x)$ when $[f, g](x) \neq 0$. Moreover, if $-h^{\prime \prime}+q h=$ $\gamma h$, then, setting

$$
\hat{h}=\frac{f}{[f, g]}\left[\frac{[f, g]}{f}, \frac{[f, h]}{f}\right]=(\gamma-\alpha) h+(\alpha-\beta) \frac{[f, h]}{[f, g]} g,
$$

one has $-\hat{h}^{\prime \prime}(x)+\hat{q}(x) \hat{h}(x)=\gamma \hat{h}(x)$ when $[f, g](x) \neq 0$. As we will see, it is possible to choose $f$ and $g$ in the cases we require so that $[f, g](x) \neq 0$ on $[0,1]$.

Given $(q, B)$, we let $\mu_{n}(t)$ be the solution of (2.13). To integrate $V_{n}$, we must choose $f$ and $g$ and $\hat{B}$ so that

$$
\begin{aligned}
\mu_{m}(\hat{q}, \hat{B}) & = \begin{cases}\mu_{m}(q, B), & m \neq n, \\
\mu_{n}(t), & m=n\end{cases} \\
y_{2}\left(1, \mu_{n}(\hat{q}, \hat{B}), \hat{q}\right) & = \begin{cases}y_{2}\left(1, \mu_{m}(q, B), q\right), & m \neq n, \\
-b / \xi_{*}\left(\mu_{n}(t)\right), & m=n .\end{cases}
\end{aligned}
$$

The correct choices turn out to be

$$
f=y_{2}\left(x, \mu_{n}, q\right), \quad g=f_{*}\left(x, \mu_{n}(t), q, B\right),
$$

where once again $*= \pm$ is determined by (2.15). Taking (2.17) with these choices of $f$ and $g$ as the definition of $\hat{q}$, we need to show that (2.17) does define a potential in $L^{2}[0,1]$, choose $\hat{B}$ and then show that (2.19) holds.

LEMMA 2.1. $\left[y_{2}\left(\mu_{n}, f_{*}(\lambda)\right](x)<0\right.$ for $(x, \lambda) \in[0,1] \times\left[\lambda_{2 n-1}, \lambda_{2 n}\right]$.

Proof. This proof follows the proof of lemma 6.1 in [6] closely. We have

$$
\left[y_{2}\left(\mu_{n}\right), f_{*}(\lambda)\right](0)=-1, \quad\left[y_{2}\left(\mu_{n}\right), f_{*}(\lambda)\right](1)=\frac{y_{2}\left(\mu_{n}\right)}{b} \xi_{*}(\lambda)
$$

Since $\Delta\left(\mu_{n}\right)=-b / y_{2}\left(1, \mu_{n}\right)-y_{2}\left(1, \mu_{n}\right) / b$, we have $\xi_{*}\left(\mu_{n}\right)=-b / y_{2}\left(1, \mu_{n}\right)=\xi_{1}\left(\mu_{n}\right)$ or $\xi_{*}\left(\mu_{n}\right)=-y_{2}\left(1, \mu_{n}\right) / b=\xi_{2}\left(\mu_{n}\right)$. Thus $\left[y_{2}\left(\mu_{n}\right), f_{*}(\lambda)\right](x)<0$ for $\lambda \in\left[\lambda_{2 n-1}, \lambda_{2 n}\right]$ when $x=0$ or 1 .

Since $a y_{1}\left(1, \mu_{n}\right)+b y_{1}^{\prime}\left(1, \mu_{n}\right)=-b / y_{2}\left(1, \mu_{n}\right)$,

$$
\lim _{\lambda \rightarrow \mu_{n}}\left[y_{2}\left(\mu_{n}\right), f_{*}(\lambda)\right](x)=-1-\frac{\xi_{*}\left(\mu_{n}\right)+b y_{2}^{-1}\left(1, \mu_{n}\right)}{a \frac{\partial y_{2}}{\partial \mu}\left(1, \mu_{n}\right)+b \frac{\partial y_{2}^{\prime}}{\partial \mu}\left(1, \mu_{n}\right)} \int_{0}^{x} y_{2}^{2}\left(x, \mu_{n}\right) d x
$$

(see (2.8)). Thus the limit is monotone in $x$ and by the preceding paragraph we conclude

$$
\lim _{\lambda \rightarrow \mu_{n}}\left[y_{2}\left(\mu_{n}\right), f_{*}(\lambda)\right](x)<0
$$

for $x \in[0,1]$.

Let $\bar{\mu}=\sup \left\{\mu^{\prime} \leq \lambda_{2 n}: \quad\left[y_{2}\left(\mu_{n}\right), f_{*}(\mu)\right]<0 \quad\right.$ for $\left.\quad(x, \mu) \in[0,1] \times\left[\mu_{n}, \mu^{\prime}\right]\right\}$. If $\left[y_{2}\left(\mu_{n}\right), f_{*}(\bar{\mu})\right]<0$ for $x \in[0,1], \bar{\mu}=\lambda_{2 n}$ and we have part of the conclusion. If $\left[y_{2}\left(\mu_{n}, f_{*}(\bar{\mu})\right]\left(x_{0}\right)=0\right.$, then

$$
x_{0} \in(0,1), \quad 0=\left[y_{2}\left(\mu_{n}\right), f_{*}(\bar{\mu})\right]^{\prime}\left(x_{0}\right)=\left(\bar{\mu}-\mu_{n}\right) y_{2}\left(x_{0}, \mu_{n}\right) f_{*}\left(x_{0}, \bar{\mu}\right),
$$

and $\left[y_{2}\left(\mu_{n}\right), f_{*}(\bar{\mu})\right](x)$ does not change sign at $x=x_{0}$. From $\left[y_{2}\left(\mu_{n}\right), f_{*}(\bar{\mu})\right]\left(x_{0}\right)=0$ and either $y_{2}\left(x_{0}, \mu_{n}\right)=0$ or $f_{*}\left(x_{0}, \bar{\mu}\right)=0$, it follows that $y_{2}\left(x_{0}, \mu_{n}\right)=f_{*}\left(x_{0}, \bar{\mu}\right)=0$. 
Since both these zeros must be simple, it follows that

$$
\left[y_{2}\left(\mu_{n}\right), f_{x}(\bar{\mu})\right](x)=k\left(x-x_{0}\right)^{3}+O\left(\left(x-x_{0}\right)^{4}\right)
$$

with $k \neq 0$. This contradicts $\left[y_{2}\left(\mu_{n}\right), f_{*}(\bar{\mu})\right](x)$ being of one sign on $[0,1]$ and we conclude

$$
\left[y_{2}\left(\mu_{n}\right), f_{*}(\lambda)\right](x)<0
$$

for $(x, \lambda) \in[0,1] \times\left[\mu_{n}, \lambda_{2 n}\right]$. The argument for $\lambda \in\left[\lambda_{2 n-1}, \mu_{n}\right]$ is identical.

With lemma 2.1 we know that for $\hat{\mu}_{n} \in\left[\lambda_{2 n-1}, \lambda_{2 n}\right]$

$$
\hat{q}=q-2 \frac{d^{2}}{d x^{2}} \log \left[f_{*}\left(\hat{\mu}_{n}\right), y_{2}\left(\mu_{n}\right)\right]
$$

is a well defined potential on $[0,1]: \hat{q}-q$ is absolutely continuous. Thus by (2.17)

$$
y(x)=\frac{y_{2}\left(x, \hat{\mu}_{n}, q\right)}{\left[f_{*}\left(\hat{\mu}_{n}\right), y_{2}\left(\mu_{n}\right)\right]}
$$

satisfies $-y^{\prime \prime}+\hat{q} y=\hat{\mu}_{n} y$. Since $y(0)=0$ and $y^{\prime}(0)=1$, we see

$$
y(x)=y_{2}\left(x, \hat{\mu}_{n}, \hat{q}\right) \text {. }
$$

To choose $\hat{B}$ so that $\mu_{n}(\hat{q}, \hat{B})=\hat{\mu}_{n}$, we need to pick $\hat{a}$ so that

$$
\hat{a} y_{2}\left(1, \hat{\mu}_{n}, \hat{q}\right)+b y_{2}^{\prime}\left(1, \hat{\mu}_{n}, \hat{q}\right)=0 \text {. }
$$

We have

$$
\begin{aligned}
y_{2}\left(1, \hat{\mu}_{n}, \hat{q}\right) & =-b / \xi_{*}\left(\hat{\mu}_{n}\right), \\
y_{2}^{\prime}\left(1, \hat{\mu}_{n}, \hat{q}\right) & =\frac{y_{2}^{\prime}\left(1, \mu_{n}, q\right)}{\left(\frac{-y_{2}\left(1, \mu_{n}, q\right)}{b} \xi_{*}\left(\hat{\mu}_{n}\right)\right)}-\frac{y_{2}^{2}\left(1, \mu_{n}, q\right)\left(\hat{\mu}_{n}-\mu_{n}\right) f_{*}\left(1, \hat{\mu}_{n}, q\right)}{\left(\frac{y_{2}\left(1, \mu_{n}, q\right)}{b} \xi_{*}\left(\hat{\mu}_{n}\right)\right)^{2}} \\
& =\frac{a}{\xi_{*}\left(\hat{\mu}_{n}\right)}-\frac{\left(\hat{\mu}_{n}-\mu_{n}\right) b^{2} f_{*}\left(1, \hat{\mu}_{n}, q\right)}{\xi_{*}^{2}\left(\hat{\mu}_{n}\right)} .
\end{aligned}
$$

Thus

$$
\hat{a} y_{2}\left(1, \hat{\mu}_{n}, \hat{q}\right)+b y_{2}^{\prime}\left(1, \hat{\mu}_{n}, \hat{q}\right)=\frac{1}{\xi_{*}\left(\hat{\mu}_{n}\right)}\left(-\hat{a} b+a b-\left(\hat{\mu}_{n}-\mu_{n}\right) \frac{b^{3} f_{*}\left(1, \hat{\mu}_{n}, q\right)}{\xi_{*}\left(\hat{\mu}_{n}\right)}\right)
$$

Thus we require

$$
\begin{aligned}
\hat{a} & =a-\left(\hat{\mu}_{n}-\mu_{n}\right) \frac{b^{2} f_{*}\left(1, \hat{\mu}_{n}, q\right)}{\xi_{*}\left(\hat{\mu}_{n}\right)} \\
& =a-\left(\hat{\mu}_{n}-\mu_{n}\right) b^{2} \frac{y_{2}\left(1, \hat{\mu}_{n}, q\right)+b / \xi_{*}\left(\hat{\mu}_{n}\right)}{a y_{2}\left(1, \hat{\mu}_{n}, q\right)+b y_{2}^{\prime}\left(1, \hat{\mu}_{n}, q\right)}
\end{aligned}
$$

and define

$$
\hat{B}=\left(\begin{array}{ll}
\hat{a} & b \\
\hat{c} & d
\end{array}\right),
$$

where $\hat{c}$ is determined by $\hat{a} d-b \hat{c}=1$.

LEMMA 2.2. $\mu_{m}(\hat{q}, \hat{B})$ and $y_{2}\left(1, \mu_{m}(\hat{q}, \hat{B}), \hat{q}\right)$ are given by $(2.19)$ for all $m$. 
Proof. By (2.18), since $\left[y_{2}\left(\mu_{n}\right), y_{2}(\lambda)\right]$ vanishes to second order at $x=0$, we have

$$
y_{2}(x, \lambda, \hat{q})=y_{2}(x, \lambda, q)+\frac{\mu_{n}-\hat{\mu}_{n}}{\lambda-\mu_{n}} \frac{\left[y_{2}\left(\mu_{n}\right), y_{2}(\lambda)\right]}{\left[y_{2}\left(\mu_{n}\right), f_{*}\left(\hat{\mu}_{n}\right)\right]} f_{*}\left(x, \hat{\mu}_{n}, q\right) \text {. }
$$

Hence

$$
y_{2}(1, \lambda, \hat{q})=y_{2}(1, \lambda, q)+\frac{\mu_{n}-\hat{\mu}_{n}}{\lambda-\mu_{n}} \frac{a y_{2}(1, \lambda, q)+b y_{2}^{\prime}(1, \lambda, q)}{\xi_{*}\left(\hat{\mu}_{n}\right)} f_{*}(1, \hat{\mu}, q)
$$

and

$$
\begin{aligned}
y_{2}^{\prime}(1, \lambda, \hat{q})= & y_{2}^{\prime}(1, \lambda, q)+\frac{\mu_{n}-\hat{\mu}_{n}}{\lambda-\mu_{n}}\left(\frac{b\left(\mu_{n}-\lambda\right) y_{2}(1, \lambda, q)}{\xi_{*}\left(\hat{\mu}_{n}\right)} f_{*}\left(1, \hat{\mu}_{n}, q\right)\right. \\
& +\frac{a y_{2}(1, \lambda, q)+b y_{2}^{\prime}(1, \lambda, q)}{\xi_{*}\left(\hat{\mu}_{n}\right)} f_{*}^{\prime}\left(1, \hat{\mu}_{n}, q\right) \\
& \left.-\frac{b\left(a y_{2}(1, \lambda, q)+b y_{2}^{\prime}(1, \lambda, q)\right)}{y_{2}\left(1, \mu_{n}, q\right) \xi_{*}\left(\hat{\mu}_{n}\right)^{2}}\left[y_{2}\left(\mu_{n}\right), f_{*}\left(\hat{\mu}_{n}\right)\right]^{\prime}(1) f_{*}\left(1, \hat{\mu}_{n}\right)\right) .
\end{aligned}
$$

Thus, since $a y_{2}\left(1, \mu_{m}, q\right)+b y_{2}^{\prime}\left(1, \mu_{m}, q\right)=0$, it follows directly from (2.20) that for $m \neq n$

$$
\hat{a} y_{2}\left(1, \mu_{m}, \hat{q}\right)+b y_{2}^{\prime}\left(1, \mu_{m}, \hat{q}\right)=0
$$

In $\S 1$ we showed

$$
\mu_{m}=\left(m-\frac{1}{2}\right)^{2} \pi^{2}+O(1) .
$$

Thus $\mu_{m}$ must actually be the $m$ th zero of $\hat{a} y_{2}(1, \lambda, \hat{q})+b y_{2}(1, \lambda, \hat{q})$ for $m$ large. Consequently, this function has no zeros other than $\{\mu\}_{m \neq n}$ and $\hat{\mu}_{n}$. Thus

$$
\mu_{m}(\hat{q}, \hat{B})=\left\{\begin{array}{cc}
\mu_{m}(q, B), & m \neq n, \\
\hat{\mu}_{n}, & m=n
\end{array} .\right.
$$

Finally we note that $(2.21)$ implies $y_{2}\left(1, \mu_{m}, \hat{q}\right)=y_{2}\left(1, \mu_{m}, q\right)$ for $m \neq n$ and $y_{2}\left(1, \hat{\mu}_{n}, \hat{q}\right)=-b / \xi_{*}\left(\hat{\mu}_{n}\right)$.

Combining theorem 2.2, lemma 2.2 and theorem 1.5, we have proven:

TheOREM 2.3. The solution to $(\dot{q}, \dot{B})=V_{n}(q, B)$ with initial data $q(0)=q, B(0)=B$ is given by

$$
\begin{aligned}
q(t) & =q-2 \frac{d^{2}}{d x^{2}} \log \left[f_{*}\left(\mu_{n}(t), q\right), y_{2}\left(\mu_{n}, q\right)\right], \\
B(t) & =\left(\begin{array}{ll}
a-\left(\mu_{n}(t)-\mu_{n}\right) b^{2} \frac{f_{*}\left(1, \mu_{n}(t), q\right)}{\xi_{*}\left(\mu_{n}(t)\right)} & b \\
c-\left(\mu_{n}(t)-\mu_{n}\right) b d \frac{f_{*}\left(1, \mu_{n}(t), q\right)}{\xi_{*}\left(\mu_{n}(t)\right)} & d
\end{array}\right),
\end{aligned}
$$

where $\mu_{n}=\mu_{n}(q, B)$, and $\mu_{n}(t)$ and $*$ are determined from (2.13) and (2.15).

The formula in theorem 2.3 may be iterated to give the result of moving successively on integral curves of $V_{n_{1}}, \ldots, V_{n_{k}}$. Without further computation this would involve $y_{2}\left(x, \lambda, q_{i}\right)$ and $f_{*}\left(x, \lambda, q_{i}, B_{i}\right), i=0, \ldots, k-1$, where $\left(q_{i}, B_{i}\right)$ is the result of moving $\left(q_{i-1}, B_{i-1}\right)$ along an integral curve of $V_{i}$. However, since the flows commute, it is 
possible to derive manageable expressions for the final $(q, B)$ in terms of $y_{2}\left(x, \lambda, q_{0}\right)$ and $f_{*}\left(x, \lambda, q_{0}, B_{0}\right)$.

To derive the formula described in the preceding paragraph, we begin by computing the analogue of $(2.21)$ for $f_{*}(x, \lambda, \hat{q}, \hat{B})$. For $\mu_{n}, \hat{\mu}$ and $\lambda$ distinct, and $\sigma= \pm$, $\delta= \pm$, let

$$
f(x)=\frac{\lambda-\mu_{n}}{\lambda-\hat{\mu}_{n}} f_{\sigma}(x, \lambda, q, B)+\frac{\mu_{n}-\hat{\mu}_{n}}{\lambda-\hat{\mu}_{n}} \frac{\left[y_{2}\left(\mu_{n}\right), f_{\sigma}(\lambda)\right]}{\left[y_{2}\left(\mu_{n}\right), f_{\delta}\left(\hat{\mu}_{n}\right)\right]} f_{\delta}\left(x, \hat{\mu}_{n}, q, B\right) .
$$

Note that $f(0)=1$ and by $(2.17)$

$$
-f^{\prime \prime}+\hat{q} f=\lambda f .
$$

Thus to show that $f(x)=f_{\sigma}(x, \lambda, \hat{q})$ it suffices to show

$$
\hat{B}\left(\begin{array}{c}
f(1) \\
f^{\prime}(1)
\end{array}\right)=\xi_{\sigma}(\lambda)\left(\begin{array}{c}
f(0) \\
f^{\prime}(0)
\end{array}\right) .
$$

We will do this without assuming $\Delta(\lambda, \hat{q}, \hat{B})=\Delta(\lambda, q, B)$, hence proving $(\hat{q}, B)$ is isospectral to $(q, B)$ without using theorem 2.3. We have, with $\xi_{\tau}(\lambda)$ equal to the root of $\xi^{2}-\Delta(\lambda, q, B) \xi+1=0$ used in defining $f_{\tau}(x, \lambda, q, B)$,

$$
\begin{aligned}
\hat{B}\left(\begin{array}{c}
f(1) \\
f^{\prime}(1)
\end{array}\right)= & \left(\frac{\lambda-\mu_{n}}{\lambda-\hat{\mu}_{n}}\right) \hat{B}\left(\begin{array}{l}
f_{\sigma}(1, \lambda) \\
f_{\sigma}^{\prime}(1, \lambda)
\end{array}\right)+\left(\frac{\mu_{n}-\hat{\mu}_{n}}{\lambda-\hat{\mu}_{n}}\right)\left(\frac{\xi_{\sigma}(\lambda)}{\xi_{\delta}\left(\hat{\mu}_{n}\right)}\right) \hat{B}\left(\begin{array}{l}
f_{\delta}\left(1, \hat{\mu}_{n}\right) \\
f_{\delta}^{\prime}\left(1, \hat{\mu}_{n}\right)
\end{array}\right) \\
& +\left(\frac{\mu_{n}-\hat{\mu}_{n}}{\lambda-\hat{\mu}_{n}}\right)\left(\frac{\left[y_{2}\left(\mu_{n}\right), f_{\sigma}(\lambda)\right]}{\left[y_{2}\left(\mu_{n}\right), f_{\delta}\left(\hat{\mu}_{n}\right)\right]}\right)^{\prime}(1) \hat{B}\left(\begin{array}{c}
0 \\
f_{\delta}\left(1, \hat{\mu}_{n}\right)
\end{array}\right) .
\end{aligned}
$$

Hence, since $\left[y_{2}(\beta), f_{*}(\lambda)\right](0)=1$,

$$
\begin{aligned}
\hat{B}\left(\begin{array}{l}
f(1) \\
f^{\prime}(1)
\end{array}\right)-\xi_{\sigma}(\lambda)\left(\begin{array}{c}
f(0) \\
f^{\prime}(0)
\end{array}\right) & \\
= & \left(\begin{array}{ll}
\hat{a}-a & 0 \\
\hat{c}-c & 0
\end{array}\right)\left[\left(\frac{\lambda-\mu_{n}}{\lambda-\hat{\mu}_{n}}\right)\left(\begin{array}{l}
f_{\sigma}(1, \lambda) \\
f_{\sigma}^{\prime}(1, \lambda)
\end{array}\right)+\left(\frac{\mu_{n}-\hat{\mu}_{n}}{\lambda-\hat{\mu}_{n}}\right)\left(\frac{\xi_{\sigma}(\lambda)}{\xi_{\delta}\left(\hat{\mu}_{n}\right)}\right)\left(\begin{array}{l}
f_{\delta}\left(1, \hat{\mu}_{n}\right) \\
f_{\delta}^{\prime}\left(1, \hat{\mu}_{n}\right)
\end{array}\right)\right] \\
& +\left(\frac{\mu_{n}-\hat{\mu}_{n}}{\lambda-\hat{\mu}_{n}}\right)\left(\frac{\left(\mu_{n}-\lambda\right) f_{\sigma}(1, \lambda) \xi_{\delta}\left(\hat{\mu}_{n}\right)-\left(\mu_{n}-\hat{\mu}_{n}\right) f_{\delta}\left(1, \hat{\mu}_{n}\right) \xi_{\sigma}(\lambda)}{\left(\xi_{\delta}\left(\hat{\mu}_{n}\right)\right)^{2}}\right) \\
& \times\left(\begin{array}{l}
b^{2} f_{\delta}\left(1, \hat{\mu}_{n}\right) \\
b d f_{\delta}\left(1, \hat{\mu}_{n}\right)
\end{array}\right) .
\end{aligned}
$$

Since

$$
\left(\begin{array}{l}
\hat{a}-a \\
\hat{c}-c
\end{array}\right)=\left(\begin{array}{l}
\left(\mu_{n}-\hat{\mu}_{n}\right) b^{2} \frac{f_{\delta}\left(1, \hat{\mu}_{n}\right)}{\xi_{\delta}\left(\hat{\mu}_{n}\right)} \\
\left(\mu_{n}-\hat{\mu}_{n}\right) b d \frac{f_{\delta}\left(1, \hat{\mu}_{n}\right)}{\xi_{\delta}\left(\hat{\mu}_{n}\right)}
\end{array}\right),
$$


we have

$$
\hat{B}\left(\begin{array}{c}
f(1) \\
f^{\prime}(1)
\end{array}\right)-\xi_{\sigma}(\lambda)\left(\begin{array}{c}
f(0) \\
f^{\prime}(0)
\end{array}\right)=\left(\begin{array}{l}
0 \\
0
\end{array}\right)
$$

as claimed. Thus

$$
f_{\sigma}(x, \lambda, \hat{q}, \hat{B})=\left(\frac{\lambda-\mu_{n}}{\lambda-\hat{\mu}_{n}}\right) f_{\sigma}(x, \lambda, q, B)+\left(\frac{\mu_{n}-\hat{\mu}_{n}}{\lambda-\hat{\mu}_{n}}\right) \frac{\left[y_{2}\left(\mu_{n}\right), f_{\sigma}(\lambda)\right]}{\left[y_{2}\left(\mu_{n}\right), f_{\delta}\left(\hat{\mu}_{n}\right)\right]} f_{\delta}\left(x, \hat{\mu}_{n}, q, B\right) \text {. }
$$

To get a compact formula for the result of moving $\mu_{k}$ to $\hat{\mu}_{k}, k=1, \ldots, N$, we will need a compact formula for $\left[y_{2}(\alpha, \hat{q}), f_{*}(\beta, \hat{q}, \hat{B})\right]$. For this we note first

$$
f_{*}\left(x, \hat{\mu}_{n}\right)\left[y_{2}\left(\mu_{n}\right), y_{2}(\alpha)\right]=y_{2}(x, \alpha)\left[y_{2}\left(\mu_{n}\right), f_{*}\left(\hat{\mu}_{n}\right)\right]-y_{2}\left(x, \mu_{n}\right)\left[y_{2}(\alpha), f_{*}\left(\hat{\mu}_{n}\right)\right] .
$$

Hence (2.21) may be rewritten

$$
y_{2}(x, \alpha, \hat{q})=\left(\frac{\alpha-\hat{\mu}_{n}}{\alpha-\mu_{n}}\right) y_{2}(x, \alpha, q)+\left(\frac{\hat{\mu}_{n}-\mu_{n}}{\alpha-\mu_{n}}\right) \frac{\left[y_{2}\left(\alpha, f_{*}\left(\hat{\mu}_{n}\right)\right]\right.}{\left[y_{2}\left(\mu_{n}, f_{*}\left(\hat{\mu}_{n}\right)\right]\right.} y_{2}\left(x, \mu_{n}, q\right) .
$$

Next by (2.22) and (2.23)

$$
\begin{aligned}
& {\left[y_{2}(\alpha, \hat{q}), f_{\sigma}(\beta, \hat{q}, \hat{B})\right]^{\prime}=(\alpha-\beta) y_{2}(x, \alpha, \hat{q}) f_{\sigma}(x, \beta, \hat{q}, \hat{B}) } \\
&=(\alpha-\beta)\left[\left(\frac{\alpha-\hat{\mu}_{n}}{\alpha-\mu_{n}}\right)\left(\frac{\beta-\mu_{n}}{\beta-\hat{\mu}_{n}}\right) y_{2}(x, \alpha) f_{\sigma}(x, \beta)\right. \\
& \quad+\left(\frac{\alpha-\hat{\mu}_{n}}{\alpha-\mu_{n}}\right)\left(\frac{\mu_{n}-\hat{\mu}_{n}}{\beta-\hat{\mu}_{n}}\right) \frac{\left[y_{2}\left(\mu_{n}\right), f_{\sigma}(\beta)\right]}{\left[y_{2}\left(\mu_{n}\right), f_{\delta}\left(\hat{\mu}_{n}\right)\right]} y_{2}(x, \alpha) f_{\delta}\left(x, \hat{\mu}_{n}\right) \\
&+\left(\frac{\hat{\mu}_{n}-\mu_{n}}{\alpha-\mu_{n}}\right)\left(\frac{\beta-\mu_{n}}{\beta-\hat{\mu}_{n}}\right) \frac{\left[y_{2}(\alpha), f_{\delta}\left(\hat{\mu}_{n}\right)\right]}{\left[y_{2}\left(\mu_{n}\right), f_{\delta}\left(\hat{\mu}_{n}\right)\right]} y_{2}\left(x, \mu_{n}\right) f_{\sigma}(x, \beta) \\
&\left.+\left(\frac{\hat{\mu}_{n}-\mu_{n}}{\alpha-\mu_{n}}\right)\left(\frac{\mu_{n}-\hat{\mu}_{n}}{\beta-\hat{\mu}_{n}}\right) \frac{\left[y_{2}\left(\mu_{n}\right), f_{\sigma}(\beta)\right]\left[y_{2}(\alpha), f_{\delta}\left(\hat{\mu}_{n}\right)\right]}{\left[y_{2}\left(\mu_{n}\right), f_{\delta}\left(\hat{\mu}_{n}\right)\right]^{2}} y_{2}\left(x, \mu_{n}\right) f_{\delta}\left(x, \hat{\mu}_{n}\right)\right] \\
&= {\left[\left(\frac{\alpha-\hat{\mu}_{n}}{\alpha-\mu_{n}}\right)\left(\frac{\beta-\mu_{n}}{\beta-\hat{\mu}_{n}}\right)\left[y_{2}(\alpha), f_{\sigma}(\beta)\right]\right.} \\
&\left.+\left(\frac{\alpha-\beta}{\alpha-\mu_{n}}\right)\left(\frac{\mu_{n}-\hat{\mu}_{n}}{\beta-\hat{\mu}_{n}}\right) \frac{\left[y_{2}\left(\mu_{n}\right), f_{\sigma}(\beta)\right]\left[y_{2}(\alpha), f_{\delta}\left(\hat{\mu}_{n}\right)\right]}{\left[y_{2}\left(\mu_{n}\right), f_{\delta}\left(\hat{\mu}_{n}\right)\right]}\right]
\end{aligned}
$$

and we conclude

$$
\begin{aligned}
& {\left[y_{2}(\alpha, \hat{q}), f_{\sigma}(\beta, \hat{q}, \hat{B})\right]} \\
& \quad=\left(\frac{\alpha-\hat{\mu}_{n}}{\alpha-\mu_{n}}\right)\left(\frac{\beta-\mu_{n}}{\beta-\hat{\mu}_{n}}\right)\left[y_{2}(\alpha, q), f_{\sigma}(\beta, q, \beta)\right] \\
& \quad+\left(\frac{\alpha-\beta}{\alpha-\mu_{n}}\right)\left(\frac{\mu_{n}-\hat{\mu}_{n}}{\beta-\hat{\mu}_{n}}\right) \frac{\left[y_{2}\left(\mu_{n}, q\right), f_{\sigma}(\beta, q, \beta)\right]\left[y_{2}(\alpha, q), f_{\delta}\left(\hat{\mu}_{n}, q, B\right)\right]}{\left[y_{2}\left(\mu_{n}, q\right), f_{\delta}\left(\hat{\mu}_{n}, q, B\right)\right]},
\end{aligned}
$$

since both sides of this equation equal -1 at $x=0$.

Theorem 2.4. Assume $\mu_{k} \neq \hat{\mu}_{k}$ for $k=1, \ldots, N$. Let

$$
\theta_{j k}(q, B)=\frac{1}{\mu_{j}-\hat{\mu}_{k}}\left[y_{2}\left(\mu_{j}, q\right), f_{\sigma_{k}}\left(\hat{\mu}_{k}, q, B\right)\right]
$$


and let $\theta(q, B)$ be the matrix with entries $\theta_{j k}$. Using the flow of $V_{k}, k=1, \ldots, N$, to move $\mu_{k}$ to $\hat{\mu}_{k}$ and $y_{2}\left(1, \mu_{k}, q\right)$ to $-b \xi_{\sigma_{k}}^{-1}\left(\hat{\mu}_{k}\right), i=1, \ldots, N$, one reaches $(\hat{q}, \hat{B})$, where

$$
\begin{aligned}
& \hat{q}=q-2 \frac{d^{2}}{d x^{2}} \log |\operatorname{det} \theta(q, B)|, \\
& \hat{a}=a-\sum_{k=1}^{N} b^{2} \prod_{l \neq k}\left(\frac{\hat{\mu}_{k}-\mu_{l}}{\hat{\mu}_{k}-\hat{\mu_{l}}}\right)\left(\hat{\mu}_{k}-\mu_{k}\right) \frac{f_{\sigma_{k}}\left(1, \hat{\mu}_{k}, q, B\right)}{\xi_{\sigma_{k}}\left(\hat{\mu}_{k}\right)}, \\
& \hat{c}=c-\sum_{k=1}^{N} b d \prod_{l \neq k}\left(\frac{\hat{\mu}_{k}-\mu_{l}}{\hat{\mu}_{k}-\hat{\mu}_{l}}\right)\left(\hat{\mu}_{k}-\mu_{k}\right) \frac{f_{\sigma_{k}}\left(1, \hat{\mu}_{k}, q, B\right)}{\xi_{\sigma_{k}}\left(\hat{\mu}_{k}\right)},
\end{aligned}
$$

$\hat{d}=d$ and $\hat{b}=b$.

Proof. Letting $\left(q_{0}, B_{0}\right)=(q, B)$ and $\left(q_{m}, B_{m}\right)$ be the result of moving from $\left(q_{m-1}, B_{m-1}\right)$ in the flow of $V_{k}$, formula $(2.24)$ implies for $j, k \neq m$

$$
\begin{aligned}
& \frac{\left(\mu_{j}-\mu_{m}\right)\left(\hat{\mu}_{k}-\hat{\mu}_{m}\right)}{\left(\mu_{j}-\hat{\mu}_{m}\right)\left(\hat{\mu}_{k}-\mu_{m}\right)} \theta_{j k}\left(q_{m}, B_{m}\right) \\
& \quad=\theta_{j k}\left(q_{m-1}, B_{m-1}\right)-\frac{\theta_{j m}\left(q_{m-1}, B_{m-1}\right) \theta_{m k}\left(q_{m-1}, B_{m-1}\right)}{\theta_{m m}\left(q_{m-1}, B_{m-1}\right)} .
\end{aligned}
$$

Consider the process of reducing $\theta$ to upper triangular form by Gauss elimination. Letting $\theta_{j k}\left(q_{m}, B_{m}\right)=\theta_{j k}(m)$ and

$$
c_{j k}(m)=\frac{\left(\mu_{j}-\mu_{m}\right)\left(\hat{\mu}_{k}-\hat{\mu}_{m}\right)}{\left(\mu_{j}-\hat{\mu}_{m}\right)\left(\hat{\mu}_{k}-\mu_{m}\right)}=a_{j}(m) b_{k}(m),
$$

after the entries below the diagonal in the first column have been cancelled, $(2.25)$ implies

$$
\begin{aligned}
\operatorname{det} \theta & =\operatorname{det}\left(\begin{array}{cccc}
\theta_{11}(0) & & \cdots & \theta_{1 N}(0) \\
0 & c_{22}(1) \theta_{22}(1) & \cdots & c_{2 N}(1) \theta_{2 N}(1) \\
\vdots & \vdots & & \vdots \\
0 & c_{N 2}(1) \theta_{N 2}(1) & \cdots & c_{N N}(1) \theta_{N N}(1)
\end{array}\right) \\
& =\prod_{j=2}^{N} a_{j}(1) \prod_{k=2}^{N} b_{k}(1) \operatorname{det}\left(\begin{array}{cccc}
\theta_{11}(0) & & \cdots & \theta_{1 N}(0) \\
0 & \theta_{22}(1) & \cdots & \theta_{2 N}(1) \\
\vdots & \vdots & & \vdots \\
0 & \theta_{N 2}(1) & \cdots & \theta_{N N}(1)
\end{array}\right) .
\end{aligned}
$$

Thus, proceeding in the same manner,

$$
\operatorname{det} \theta=\left(\prod_{m=1}^{N-1} \prod_{j=m+1}^{N} a_{j}(m) \prod_{k=m+1}^{N} b_{k}(m)\right) \prod_{k=1}^{N} \theta_{k k}(k-1) .
$$

Since theorem 2.3 implies

$$
\hat{q}=q-\sum_{k=1}^{N} 2 \frac{d^{2}}{d x^{2}} \log \left|\theta_{k k}\left(q_{k-1}, B_{k-1}\right)\right|,
$$

we have proven the formula for $\hat{q}$ in theorem 2.4 .

To prove the formula for $\hat{B}$ in theorem 2.4 , it clearly suffices to prove the formula for $\hat{a}$ and we will use induction on $N$ for that. In the case $N=1$ the formula reduces 
to (2.20). Assuming the formula for $N=M-1$ and applying it to $\left(q_{1}, B_{1}\right)$,

$$
\hat{a}=a_{1}-\sum_{k=2}^{M} b^{2} \prod_{\substack{l=2 \\ l \neq k}}^{M}\left(\frac{\hat{\mu}_{k}-\mu_{l}}{\hat{\mu}_{k}-\hat{\mu}_{l}}\right)\left(\hat{\mu}_{k}-\mu_{k}\right) \frac{f_{\sigma_{k}}\left(1, \hat{\mu}_{k}, q_{1}, B_{1}\right)}{\xi_{\sigma_{k}}\left(\hat{\mu}_{k}\right)} .
$$

However, (2.22) implies for $k=2, \ldots, M$

$$
f_{\sigma_{k}}\left(1, \hat{\mu}_{k}, q_{1}, B_{1}\right)=\frac{\hat{\mu}_{k}-\mu_{1}}{\hat{\mu}_{k}-\hat{\mu}_{1}} f_{\sigma_{k}}\left(1, \hat{\mu}_{k}, q_{0}, B_{0}\right)+\frac{\hat{\mu}_{1}-\mu_{1}}{\hat{\mu}_{1}-\hat{\mu}_{k}} f_{\sigma_{1}}\left(1, \hat{\mu}_{1}, q_{0}, B_{0}\right) .
$$

Thus, using (2.20) to replace $a_{1}$ by $a_{0}$, to complete the proof of theorem 2.4 , it suffices to show

$$
1+\sum_{k=2}^{M} \frac{\hat{\mu}_{k}-\mu_{k}}{\hat{\mu}_{1}-\hat{\mu}_{k}} \prod_{l=2}^{M}\left(\frac{\hat{\mu}_{k}-\mu_{l}}{\hat{\mu}_{k}-\hat{\mu}_{l}}\right)=\prod_{l=2}^{M} \frac{\hat{\mu}_{1}-\mu_{l}}{\hat{\mu}_{1}-\hat{\mu}_{l}} .
$$

Formula (2.26) is just the statement that the sum of the residues of

$$
\frac{1}{z-\hat{\mu}_{1}} \prod_{l=2}^{M} \frac{z-\mu_{l}}{z-\hat{\mu}_{l}}=F(z)
$$

at $z=\hat{\mu}_{1}, \ldots, \hat{\mu}_{M}$ must be 1 , which follows from $\lim _{|z| \rightarrow \infty} z F(z)=1$.

Remark 2.5. In theorem 2.4 we assume $\hat{\mu}_{k} \neq \mu_{k}, k=1, \ldots, N$, since otherwise $\theta$ will be undefined. However, in theorem 2.3 the corresponding assumption, $\mu_{n} \neq \mu_{n}(t)$, is not made. While strictly speaking $\left[f_{*}\left(\mu_{n}, q\right), y_{2}\left(\mu_{n}, q\right)\right]$ is undefined, it should be clear from the computation in the proof of lemma 2.1 that in theorem 2.3 one should define

$$
\left[f_{*}\left(\mu_{n}, q\right), y_{2}\left(\mu_{n}, q\right)\right](x)=1+\frac{\xi_{*}\left(\mu_{n}\right)+b y_{2}^{-1}\left(1, \mu_{n}\right)}{a \frac{\partial y_{2}}{\partial \mu}\left(1, \mu_{n}\right)+b \frac{\partial y_{2}^{\prime}}{\partial \mu}\left(1, \mu_{n}\right)} \int_{0}^{x} y_{2}^{2}\left(x, \mu_{n}\right) d x .
$$

It is possible to extend theorem 2.4 to general $\hat{\mu}_{k}, k=1, \ldots, M$, in a similar fashion, but, since we will not have further use for $\hat{q}$, we will leave the extended formula for $\hat{q}$ to the reader. To extend the formulae for $\hat{B}$ in theorems 2.3 and 2.4 to general $\hat{\mu}_{k}$, one has only to observe that

$$
\lim _{\hat{\mu}_{k} \rightarrow \mu_{k}}\left(\hat{\mu}_{k}-\mu_{k}\right) \frac{f_{*}\left(1, \hat{\mu}_{k}\right)}{\xi_{*}\left(\hat{\mu}_{k}\right)}=\frac{y_{2}\left(1, \mu_{k}\right)+b \xi_{*}^{-1}\left(\mu_{k}\right)}{a \frac{\partial y_{2}}{\partial \mu}\left(1, \mu_{k}\right)+b \frac{\partial y_{2}^{\prime}}{\partial \mu}\left(1, \mu_{k}\right)} .
$$

\section{The manifold $M$}

In this section we give $M$ the structure of a real analytic manifold. The strategy for doing this is quite simple. In $\S 1$ we showed that the mapping $\Phi$ was an analytic homeomorphism. Elementary considerations show $\Phi(M)$ is contained in an explicit analytic submanifold $N$ of $S$. Using the flows of $\S 2$, we can show $\Phi\left(M \cap\left\{d=d_{0}\right\}\right)=$ $N \cap\left\{d=d_{0}\right\}$ when $M \cap\left\{d=d_{0}\right\} \neq \varnothing$. In $\S 5$ we will see that the range of $d$ on $M$ is $\mathbf{R}$, and we will conclude $\Phi(M)=N$. Here we only show that there is a $d_{\infty}$ so that $\Phi(M)=N \cap\left\{d<d_{\infty}\right\}$. Hence $M$ inherits its real analytic structure from $N \cap$ $\left\{d<d_{\infty}\right\}$. 
The manifold $N$ is the subset of $S$ defined by the relations

$$
\begin{gathered}
\lambda_{2 n-1} \leq \mu_{n} \leqq \lambda_{2 n}, \quad \kappa_{n}^{2}+b \Delta\left(\mu_{n}\right) \kappa_{n}+b^{2}=0, \quad n=1,2, \ldots, \\
b=b_{0}, \quad \frac{2 d}{b}+r=\frac{2\left(a_{0}+d_{0}\right)}{b}+\int_{0}^{1} q_{0} d x=I_{0},
\end{gathered}
$$

where $\left(q_{0}, B_{0}\right) \in M$. The function $r$ is the coordinate function on $S$ from (1.13). We have already seen that

$$
\Delta\left(\mu_{n}(q, B)\right)=\frac{-b}{y_{2}\left(1, \mu_{n}(q, B), q\right)}-\frac{y_{2}\left(1, \mu_{n}(q, B), q\right)}{b}
$$

(see (2.12)), so that (3.1) holds on $\Phi(M)$. Moreover, combining theorems 1.3 and 1.4, we see that (3.2) holds on $\Phi(M)$ also. Thus $\Phi(M) \subset N$.

That $N$ is an analytic submanifold of $S$ is a consequence of the properties of $\Delta$ stated in the Introduction. These imply that

(i) $\left|\Delta\left(\lambda_{k}\right)\right|=2$ for all $k$,

(ii) $|\Delta(\mu)|>2$ for $\lambda_{2 n-1}<\mu<\lambda_{2 n}$,

(iii) if $\lambda_{2 n-1}<\lambda_{2 n}$, then $\partial \Delta / \partial \lambda \neq 0$ at $\lambda_{2 n}$ or $\lambda_{2 n-1}$.

Hence for each $n$ (3.1) defines a real analytic curve-topologically a circle in the $\left(\mu_{n}, k_{n}\right)$-plane if $\lambda_{2 n-1}<\lambda_{2 n}$. When $\lambda_{2 n-1}=\lambda_{2 n}$ this curve degenerates to a point and $\left(\mu_{n}, k_{n}\right)$ is constant on $N$. By (1.6) the number of indices $n$ for which $\lambda_{2 n}=\lambda_{2 n-1}$ must be finite, and we delete them in what follows. We may give $N$ locally as an explicit submanifold in $S$ by solving

$$
\kappa_{n}^{2}+b \Delta\left(\mu_{n}\right) \kappa_{n}+b^{2}=0
$$

for $k_{n}$ or $\mu_{n}$ as is appropriate for each admissible index $n$.

Since the topology on $S$ is induced by the topology on $l_{\mathrm{R}}^{2}$ via the coordinate map $s \rightarrow \tilde{s}$, any point $s_{0} \in N$ has a neighbourhood $U$ in $S$ such that on $U$

$$
\left|\mu_{n}-\left(n-\frac{1}{2}\right)^{2} \pi^{2}-r\left(s_{0}\right)\right|<1 \quad \text { and } \quad \mid \kappa_{n}<b
$$

for $n \geq n\left(s_{0}\right)$. Hence, since $N$ will be an analytic submanifold if it is given locally as the graph of an analytic function, and the preceding remarks show we can ignore any finite set of indices in proving this analyticity, it suffices to prove the real analyticity on $V=\left\{\left\{\mu_{n}\right\}_{n>n_{1}}:\left\{b, d, \mu_{1}, \kappa_{1}, \ldots\right\} \in U\right.$ for some $\left\{b, d, \mu_{1}, \kappa_{1}, \ldots, \mu_{n_{1}}\right.$, $\left.\left.\kappa_{n_{1}} \kappa_{n_{1}+1}, \ldots\right\}\right\}$ of

$$
F:\left\{\mu_{n}\right\}_{n>n_{1}} \rightarrow\left\{\kappa_{n}\right\}_{n>n_{1}},
$$

where

$$
\kappa_{n}=(b / 2) \Delta\left(\mu_{n}\right)\left(\sqrt{1-4\left(\Delta\left(\mu_{n}\right)\right)^{-2}}-1\right)
$$

and $n_{1}>n\left(s_{0}\right)$ is chosen large enough so that on $U$ one has $\lambda_{2 n-1}<\mu_{n}<\lambda_{2 n}$ for $n \geq n_{1}$. Since each component of $F$ is clearly analytic on $V_{1}=$ $\left\{\left\{\mu_{n}\right\}_{n>n_{1}}:\left\{\operatorname{Re}\left\{\mu_{n}\right\}\right\}_{n>n_{1}} \in V,\left|\operatorname{Im}\left\{\mu_{n}\right\}\right|<1\right.$ and $\left.\left\{\operatorname{Im}\left\{\mu_{n}\right\}\right\}_{n>n_{1}} \in l^{2}\right\}$, it will suffice to 
show that the image of $V_{1}$ under $F$ is bounded, i.e. that $\left\{\left\{\tilde{\kappa}_{n}\right\}_{n>n_{1}}:\left\{\kappa_{n}\right\}_{n>n_{1}} \in F\left(V_{1}\right)\right\}$ is bounded in $l^{2}$, where $\tilde{\kappa}_{n}=n^{2}\left(\kappa_{n}+(-1)^{n} / n\right)$; cf. theorem A.3 of [6].

Showing $F$ is locally bounded requires a sharper estimate on $\Delta\left(\mu_{n}\right)$ than we have used up to now. Since, given $\left(q_{0}, B_{0}\right) \in M$,

$$
\Delta(\mu)=a_{0} y_{1}\left(1, \mu, q_{0}\right)+b y_{1}^{\prime}\left(1, \mu, q_{0}\right)+c_{0} y_{2}\left(1, \mu, q_{0}\right)+d_{0} y_{2}^{\prime}\left(1, \mu, q_{0}\right)
$$

and $\left|\sqrt{\mu_{n}}-\left(n-\frac{1}{2}\right) \pi\right|<A / n$ on $V_{1}$, one sees easily from theorem 1.1 that on $V_{1}$

$$
\left|\Delta\left(\mu_{n}\right)-b y_{1}^{\prime}\left(1, \mu_{n}, q_{0}\right)\right|<B / n \text {. }
$$

To avoid the $O(1)$ error term in the basic estimate for $y_{1}^{\prime}$, we use

$$
y_{1}^{\prime}\left(1, \mu_{n}, q_{0}\right)=\left.\frac{y_{2}^{\prime} y_{1}-1}{y_{2}}\right|_{\left(1, \mu_{n}, q_{0}\right)}=\frac{-\sin ^{2}\left(\mu_{n}^{1 / 2}\right)+\left(\cos \left(\mu_{n}^{1 / 2}\right)\right) O\left(\mu_{n}^{-1 / 2}\right)+O\left(\mu_{n}^{-1}\right)}{\mu_{n}^{-1 / 2} \sin \mu_{n}^{1 / 2}+O\left(\mu_{n}^{-1}\right)}
$$

Thus $\left|\Delta\left(\mu_{n}\right)-b \pi\left(n-\frac{1}{2}\right)(-1)^{n}\right| \leq C / n$ on $V_{1}$ and

$$
\left|\kappa_{n}\left(\mu_{n}\right)+\frac{(-1)^{n}}{\pi\left(n-\frac{1}{2}\right)}\right| \leq \frac{D}{n^{3}} \text {. }
$$

Thus, since $D$ is constant on $V_{1}$, we see $\left\{\left(\tilde{\kappa}_{n_{1}}\left(\mu_{n_{1}}\right), \ldots\right):\left(\mu_{n_{1}}, \ldots\right) \in V_{1}\right\}$ is bounded in the $l^{2}$-norm. Thus we conlude that $N$ is a real analytic submanifold of $S$.

Given $\left(q_{0}, B_{0}\right) \in M$, one sees from theorem 2.2 that by using the flows from the vector fields $V_{1}, V_{2}, \ldots$ successively, one can reach a subset of $M \cap\left\{d=d_{0}\right\}$ whose image under $\Phi$ contains all points of $N \cap\left\{d=d_{0}\right\}$ such that for $n$ sufficiently large

$$
\left(\mu_{n}, \kappa_{n}\right)=\left(\mu_{n}\left(q_{0}, B_{0}\right), y_{2}\left(1, \mu_{n}\left(q_{0}, B_{0}\right), q_{0}\right)\right) \text {. }
$$

By (3.2) on $N \cap\left\{d=d_{0}\right\}$

$$
r=I_{0}-2 d_{0} / b_{0}
$$

i.e. on $N \cap\left\{d=d_{0}\right\}$

$$
\mu_{n}=\left(n-\frac{1}{2}\right)^{2} \pi^{2}+I_{0}-2 d_{0} / b_{0}+l^{2}(n),
$$

and we see that the points of $N \cap\left\{d=d_{0}\right\}$ satisfying (3.3) for all but finitely many $n$ are dense in $N \cap\left\{d=d_{0}\right\}$. Since $\Phi^{-1}$ is continuous and $M \cap\left\{d=d_{0}\right\}$-as the intersection of closed sets-is closed in $L_{\mathbf{R}}^{2}[0,1] \times \operatorname{SL}(2, \mathbf{R})$, we have proven:

LEMMA 3.1. $\Phi\left(M \cap\left\{d=d_{0}\right\}\right)=N \cap\left\{d=d_{0}\right\}$.

To complete the argument outlined in the first paragraph of this section, we only need the following:

LEMMA 3.2. The range of $d$ on $M$ is $\left(-\infty, d_{\infty}\right)$ for some $d_{\infty} \leq \infty$.

Proof. In this lemma we make our first use of the $*$-involution. Since this involution fixes $M$ and interchanges $a$ and $d$ (cf. lemma 4.1), $a$ and $d$ have the same range on $M$. Thus we only need consider the range of $a$ on $M$. Given $(q, B) \in M$, we have from theorem 2.3

$$
a(t)=a-b^{2}\left(\mu_{n}(t)-\mu_{n}\right) \frac{y_{2}\left(1, \mu_{n}(t), q\right)+b \xi_{*}^{-1}\left(\mu_{n}(t)\right)}{y_{2}\left(1, \mu_{n}(t), q\right)+b y_{2}^{\prime}\left(1, \mu_{n}(t), q\right)}
$$

as one moves $(q, B)$ under the flow of $V_{n}$. In particular, if we move to the point 
with $\mu_{n}=\mu_{n}(q, B)$ and $\kappa_{n}=b^{2}\left(y^{2}\left(\dot{1}, \mu_{n}, q\right)\right)^{-1}$, we have (see remark 2.5)

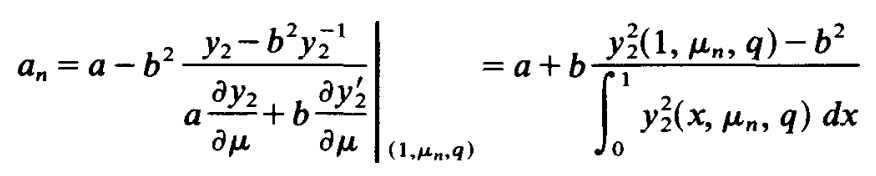

by (1.2). Since

$$
y_{2}^{2}\left(1, \mu_{n}, q\right)=\sin ^{2} \pi\left(n-\frac{1}{2}\right) x / \pi^{2}\left(n-\frac{1}{2}\right)^{2}+O\left(1 / n^{3}\right),
$$

we have

$$
a_{n}=a-2 b^{2} \pi^{2} n^{2}+O(n) .
$$

Thus $d(M)=a(M) \supset(-\infty, a]$.

Since $(q, B)$ was an arbitrary point of $M$, to complete the proof we only need show that $d$ cannot assume its least upper bound on $M$. Suppose it did assume this maximum value at $\left(q_{0}, B_{0}\right)$. Then we could choose a $\lambda_{0}$ such that $a_{0} y_{2}\left(1, \lambda_{0}, q_{0}\right)+$ $b y_{2}^{\prime}\left(1, \lambda_{0}, q_{0}\right) \neq 0$. By theorem $2.1 d \neq 0$ at $\left(q_{0}, B_{0}\right)$ on the integral curve of $V\left(q, B, \lambda_{0}\right)$ through $\left(q_{0}, B_{0}\right)$. Since this curve lies in $M$, the proof is complete.

\section{Even points}

In this section we will describe the points of $M$ which are fixed by the involution

$$
\begin{gathered}
q(x) \rightarrow q^{*}(x)=q(1-x), \\
\left(\begin{array}{ll}
a & b \\
c & d
\end{array}\right) \rightarrow\left(\begin{array}{ll}
a & b \\
c & d
\end{array}\right)^{*}=\left(\begin{array}{ll}
d & b \\
c & a
\end{array}\right)
\end{gathered}
$$

on $M$. The fixed points of this involution play the role of even potentials for Dirichlet and periodic boundary conditions and, following [4], we call them even points. These points are important when one considers the submanifold of $M, M_{B}$, obtained by fixing the boundary condition $B$.

We begin with two simple lemmas.

\section{LEMMA 4.1. The *-involution maps $M$ onto $M$.}

Proof. Suppose $-y^{\prime \prime}+q(x) y=\lambda y$ and

$$
\left(\begin{array}{ll}
a & b \\
c & d
\end{array}\right)\left(\begin{array}{l}
y(1) \\
y^{\prime}(1)
\end{array}\right)=\left(\begin{array}{l}
y(0) \\
y^{\prime}(0)
\end{array}\right) .
$$

Letting $z=y(1-x)$, we have $-z^{\prime \prime}+q^{*}(x) z=\lambda z$ and

$$
\left(\begin{array}{ll}
a & b \\
c & d
\end{array}\right)\left(\begin{array}{c}
z(0) \\
-z^{\prime}(0)
\end{array}\right)=\left(\begin{array}{c}
z(1) \\
-z^{\prime}(1)
\end{array}\right)
$$

Thus

$$
\begin{aligned}
\left(\begin{array}{l}
z(0) \\
z^{\prime}(0)
\end{array}\right) & =\left(\begin{array}{cc}
1 & 0 \\
0 & -1
\end{array}\right)\left(\begin{array}{ll}
a & b \\
c & d
\end{array}\right)^{-1}\left(\begin{array}{cc}
1 & 0 \\
0 & -1
\end{array}\right)\left(\begin{array}{c}
z(1) \\
z^{\prime}(1)
\end{array}\right) \\
& =\left(\begin{array}{ll}
d & c \\
b & a
\end{array}\right)\left(\begin{array}{l}
z(1) \\
z^{\prime}(1)
\end{array}\right)=\left(\begin{array}{ll}
a & b \\
c & d
\end{array}\right) *\left(\begin{array}{l}
z(1) \\
z^{\prime}(1)
\end{array}\right)
\end{aligned}
$$


since $\operatorname{det}\left(\begin{array}{ll}a & b \\ c & d\end{array}\right)=1$. Thus $(q, B) \in M \Rightarrow\left(q^{*}, B^{*}\right) \in M$ and, since $*$ is an involution, the proof is complete.

LEMMA 4.2. The following identities hold:

(i) $y_{1}\left(1, \lambda, q^{*}\right)=y_{2}^{\prime}(1, \lambda, q)$,

(ii) $y_{2}\left(1, \lambda, q^{*}\right)=y_{2}(1, \lambda, q)$,

(iii) $y_{1}^{\prime}\left(1, \lambda, q^{*}\right)=y_{1}^{\prime}(1, \lambda, q)$,

(iv) $y_{2}^{2}(1, \lambda, q)+b \Delta(\lambda) y_{2}(1, \lambda, q)+b^{2}$

$$
\begin{aligned}
& =\left(a y_{2}(1, \lambda, q)+b y_{2}^{\prime}(1, \lambda, q)\right)\left(a^{*} y_{2}\left(1, \lambda, q^{*}\right)+b y_{2}^{\prime}\left(1, \lambda, q^{*}\right)\right) \\
& =y_{2}^{2}\left(1, \lambda, q^{*}\right)+b \Delta(\lambda) y_{2}\left(1, \lambda, q^{*}\right)+b^{2} .
\end{aligned}
$$

We have already used (iv) in the special case $\lambda=\mu_{n}(a, b, q)$.

Proof. To verify (i)-(iii), we note that since $y_{i}\left(1-x, \lambda, q^{*}\right), i=1,2$, solves $-y^{\prime \prime}+q y=$ $\lambda y$, we must have

$$
\left(\begin{array}{l}
y_{1}\left(1-x, \lambda, q^{*}\right) \\
y_{2}\left(1-x, \lambda, q^{*}\right)
\end{array}\right)=\left(\begin{array}{ll}
\alpha_{1} & \alpha_{2} \\
\alpha_{3} & \alpha_{4}
\end{array}\right)\left(\begin{array}{l}
y_{1}(x, \lambda, q) \\
y_{2}(x, \lambda, q)
\end{array}\right)
$$

for some constants $\alpha_{i}, i=1, \ldots, 4$. Evaluating (4.1) and its derivative at $x=1$, we have

$$
\begin{aligned}
& \left(\begin{array}{c}
1 \\
0
\end{array}\right)=\left(\begin{array}{ll}
\alpha_{1} & \alpha_{2} \\
\alpha_{3} & \alpha_{4}
\end{array}\right)\left(\begin{array}{l}
y_{1}(1, \lambda, q) \\
y_{2}(1, \lambda, q)
\end{array}\right), \\
& \left(\begin{array}{c}
0 \\
-1
\end{array}\right)=\left(\begin{array}{ll}
\alpha_{1} & \alpha_{2} \\
\alpha_{3} & \alpha_{4}
\end{array}\right)\left(\begin{array}{l}
y_{1}^{\prime}(1, \lambda, q) \\
y_{2}^{\prime}(1, \lambda, q)
\end{array}\right) .
\end{aligned}
$$

Hence

$$
\left(\begin{array}{ll}
\alpha_{1} & \alpha_{2} \\
\alpha_{3} & \alpha_{4}
\end{array}\right)=\left(\begin{array}{ll}
y_{1} & -y_{1}^{\prime} \\
y_{2} & -y_{2}^{\prime}
\end{array}\right)^{-1}=\left(\begin{array}{ll}
y_{2}^{\prime}(1, \lambda, q) & -y_{1}^{\prime}(1, \lambda, q) \\
y_{2}(1, \lambda, q) & -y_{1}(1, \lambda, q)
\end{array}\right)
$$

by the Wronskian identity. Substituting for $\left(\begin{array}{cc}\alpha_{1} & \alpha_{2} \\ \alpha_{3} & \alpha_{4}\end{array}\right)$ in (4.1) and evaluating the resulting expression and its derivative at $x=0$ gives (i)-(iii).

To verify (iv), we compute as follows:

$$
\begin{aligned}
\Delta(\lambda) & =\left[a y_{1}+b y_{1}^{\prime}+c y_{2}+d y_{2}^{\prime}\right](1, \lambda, q) \\
& =\left[a y_{1}+\frac{b\left(y_{1} y_{2}^{\prime}-1\right)}{y_{2}}+c y_{2}+d y_{2}^{\prime}\right](1, \lambda, q) \\
& =\left[\frac{b a y_{1} y_{2}+b^{2} y_{1} y_{2}^{\prime}+b c y_{2}^{2}+b d y_{2} y_{2}^{\prime}-b^{2}}{b y_{2}}\right](1, \lambda, q) \\
& =\left[\frac{b a y_{1} y_{2}+b^{2} y_{1} y_{2}^{\prime}+a d y_{2}^{2}+b d y_{2} y_{2}^{\prime}}{b y_{2}}-\frac{b}{y_{2}}-\frac{y_{2}}{b}\right](1, \lambda, q) \\
& =\left[\frac{\left(a y_{2}+b y_{2}^{\prime}\right)\left(d y_{2}+b y_{1}\right)}{b y_{2}}-\frac{b}{y_{2}}-\frac{y_{2}}{b}\right](1, \lambda, q) .
\end{aligned}
$$

Now (iv) follows by multiplying by $b y_{2}$ and collecting terms.

The identity (iv) is important here because it is related to the basic formula

$$
\hat{a}=a-b^{2}\left(\tilde{\mu}_{j}-\mu_{j}\right)\left[\frac{y_{2}+b \xi_{*}}{a y_{2}+b y_{2}^{\prime}}\right]\left(1, \tilde{\mu}_{j}, q\right)
$$


of $\S 2$. By (iv), $\left[y_{2}+b \xi_{*}\right]\left(1, \tilde{\mu}_{j}, q\right)=0$ only if $\tilde{\mu}_{j}$ is a root of $\left[a y_{2}+b y_{2}^{\prime}\right](1, \lambda, q)=0$ or of $\left[d y_{2}+b y_{1}\right](1, \lambda, q)=0$. We let the roots of $\left[d y_{2}+b y_{1}\right](1, \lambda, q)=0$ be $\nu_{1}, \nu_{2}, \ldots$ Since the $\nu$ are just the $\mu$ for $\left(q^{*}, B^{*}\right)$, they have all the properties of the $\mu$ established in $\S 1$. In the next lemma we think of $y_{2}+b \xi_{*}$ as a function on

$$
\left\{(\mu, \xi): \xi^{2}-\Delta(\mu) \xi+1=0\right\},
$$

but we denote the two branches as $\xi_{ \pm}(\mu)$ as before.

LEMMA 4.3. If $\nu_{n}=\mu_{n}$ and $\lambda_{2 n-1} \neq \lambda_{2 n}$, then $\lambda_{2 n-1}<\mu_{n}<\lambda_{2 n}$ and one branch of $y_{2}+b \xi_{*}$ has a zero of order 2 in $\mu$ at $\mu=\mu_{n} \cdot y_{2}+b \xi_{*}$ has no other zeros and hence is of one sign for $\lambda_{2 n-1} \leq \mu \leq \lambda_{2 n}$. Moreover, $y_{2}+b \xi_{*}$ is of one sign for $\lambda_{2 n-1} \leq \mu \leq \lambda_{2 n}$ only if $\nu_{n}=\mu_{n}$.

Proof. By theorem 1.3, $a y_{2}+b y_{2}^{\prime}$ and $d y_{2}+b y_{1}$ have simple zeros at $\mu_{n}$ and $\nu_{n}$ for $n=1,2, \ldots$ respectively and no other zeros. The first step in this proof is showing that, if $\mu_{n}=\nu_{n}=\lambda_{2 n}$ or $\mu_{n}=\nu_{n}=\lambda_{2 n-1}$, then $\lambda_{2 n}=\lambda_{2 n-1}$. If either of these occurs, it follows that $y_{2}\left(1, \mu_{n}\right)= \pm b, y_{2}^{\prime}\left(1, \mu_{n}\right)=\mp a, y_{1}\left(1, \mu_{n}\right)=\mp d$ and thus $y_{1}^{\prime}\left(1, \mu_{n}\right)= \pm c$, since $y_{1} y_{2}^{\prime}-y_{1}^{\prime} y_{2}=1$. Hence

$$
\left(\begin{array}{ll}
a & b \\
c & d
\end{array}\right)\left(\begin{array}{ll}
y_{1}\left(1, \mu_{n}\right) & y_{2}\left(1, \mu_{n}\right) \\
y_{1}^{\prime}\left(1, \mu_{n}\right) & y_{2}^{\prime}\left(1, \mu_{n}\right)
\end{array}\right)=\left(\begin{array}{cc}
\mp 1 & 0 \\
0 & \mp 1
\end{array}\right) .
$$

Thus the generalized periodic eigenvalue at $\lambda=\mu_{n}$ has multiplicity 2 and we conclude $\lambda_{2 n-1}=\lambda_{2 n}$.

Since $\left[a y_{2}+b y_{2}^{\prime}\right](1, \lambda)$ and $\left[d y_{2}+b y_{1}\right](1, \lambda)$ both have the asymptotic form

$$
b \cos \sqrt{\lambda}+O(1 / \sqrt{\lambda})
$$

it follows that their derivatives at $\mu_{n}$ and $\nu_{n}$ respectively have the same sign. If $\nu_{n}=\mu_{n}$, it follows from lemma 4.2 (iv) that $\left[\left(y_{2}+b \xi_{+}\right)\left(y_{2}+b \xi_{-}\right)\right](1, \mu)$ is non-negative on $\left[\lambda_{2 n-1}, \lambda_{2 n}\right]$ and that it has a double zero at $\mu=\mu_{n}$ and no other zeros in $\left[\lambda_{2 n-1}, \lambda_{2 n}\right]$. Since $\xi_{+} \neq \xi_{-}$on $\left(\lambda_{2 n-1}, \lambda_{2 n}\right)$, it follows that, if $\lambda_{2 n-1}<\lambda_{2 n}$, one of the factors $y_{2}+b \xi_{ \pm}$must have a double zero at $\mu=\mu_{n}$ and the other factor is non-zero on $\left[\lambda_{2 n-1}, \lambda_{2 n}\right]$. On the other hand, if $\nu_{n} \neq \mu_{n}$, then lemma 4.2 (iv) implies [ $\left(y_{2}+\right.$ $\left.\left.b \xi_{+}\right)\left(y_{2}+b \xi_{-}\right)\right](1, \mu)$ is negative on the interval between $\nu_{n}$ and $\mu_{n}$. The lemma follows immediately.

Now we are ready to study the fixed points of the *-involution. The point of contact with the preceding discussion is the following lemma:

LEMMA 4.4. The point $(q, B)$ is fixed by the involution if and only if $\nu_{n}(q, B)=\mu_{n}(q, B)$ for all $n$.

Proof. By lemma 4.2(i) and (ii) for each $n$

$$
\nu_{n}(q, B)=\mu_{n}\left(q^{*}, B^{*}\right) \text {. }
$$

Thus the necessity of the condition is obvious.

Suppose $\mu_{n}=\nu_{n}, n=1,2, \ldots$ Since by theorem 1.3

$$
\begin{aligned}
& \mu_{n}=\left(n-\frac{1}{2}\right)^{2} \pi^{2}+2 \frac{a}{b}+\int_{0}^{1} q d x+l^{2}(n), \\
& \nu_{n}=\left(n-\frac{1}{2}\right)^{2} \pi^{2}+2 \frac{d}{b}+\int_{0}^{1} q^{*} d x+l^{2}(n),
\end{aligned}
$$


we conclude $a=d$. Since $y_{2}\left(1, \mu_{n}, q\right)=y_{2}\left(1, \mu_{n}, q^{*}\right)$ for all $n$ by lemma 4.2(i), we see $\Phi(q, B)=\Phi\left(q^{*}, B^{*}\right)$ and hence $(q, B)=\left(q^{*}, B^{*}\right)$.

Lemmas $4.2,4.3$ and 4.4 make it possible to assign an index to the fixed points of the *-involution. If $(q, B)$ is fixed, then

$$
F_{j}(\mu, \xi)=\left(\mu-\mu_{j}\right)\left(\frac{y_{2}(1, \mu, q)+b \xi}{a y_{2}(1, \mu, q)+b y_{2}^{\prime}(1, \mu, q)}\right)
$$

is of one sign on the $j$ th gap, i.e. on

$$
\left\{(\mu, \xi): \lambda_{2 j-1} \leq \mu \leq \lambda_{2 j}, 0=\xi^{2}-\Delta(\mu) \xi+1\right\} .
$$

The index of $(q, B)$ is the set $I(q, B)$ of $j$ for which $\lambda_{2 j-1}<\lambda_{j}$ and $F_{j}$ is non-positive on the $j$ th gap. This set is always finite: since $y_{2}(1, \lambda, q) \rightarrow 0$ as $\lambda \rightarrow \infty$, one sees from lemma 4.2(iv) that the sign of $F_{j}$ on the $j$ th gap for $j$ large will be the sign of $\xi_{ \pm}\left(\mu_{j}\right)\left(a \partial y_{2} / \partial \mu\left(1, \mu_{j}, q\right)+b \partial y_{2} / \partial \mu\left(1, \mu_{j}, q\right)\right)$. From (1.2) and the asymptotics of $\Delta(\lambda)$ and $y_{2}(1, \lambda)$ as $\lambda \rightarrow \infty$, one sees that this expression is positive for $j$ large.

The main result of this section is the following converse to the preceding remark:

THEOREM 4.5. For each finite subset $I$ of $Z_{+} \backslash\left\{j: \lambda_{2 j-1}=\lambda_{2 j}\right\}$ there is a unique fixed point of $*$ on $M$ of index $\mathbf{I}$.

Our strategy in proving theorem 4.5 will be to exploit the fact that the fixed points are precisely the critical points of the function $a$ on $M$. Using the mapping $\Phi$ of $\S 1$ to identify $M$ with $N \cap\left\{d<d_{\infty}\right\}$, we may consider $a$ as an analytic function on $N \cap\left\{d<d_{\infty}\right\}$. Given a finite subset $I$ of $Z_{+} \backslash\left\{j: \lambda_{2 j-1}=\lambda_{2 j}\right\}$, it is natural to split $N \cap\left\{d<d_{\infty}\right\}$ into the product of the finite-dimensional torus

$$
N_{I}=\left\{\left(\mu_{j_{1}}, \kappa_{j_{1}}, \ldots, \mu_{j_{i}}, \kappa_{j_{l}}\right): j_{i} \in I\right\}
$$

and the infinite-dimensional analytic manifold

$$
N_{I^{c}}=\left\{\left(b, d, \mu_{j_{l}}, \kappa_{j_{1}}, \ldots, \mu_{j_{l}}, \kappa_{j_{1}}, \ldots: j_{i} \in I^{c}, d<d_{\infty}\right\} .\right.
$$

The points of $N_{I}$ and $N_{I^{c}}$ are subject to (3.1) as well as, in the case of $N_{I^{c}},(3.2)$ and the asymptotic conditions defining $S$. Clearly $N \cap\left\{d<d_{\infty}\right\}=N_{I} \times N_{I^{c}}$, and we denote points of $N$ as pairs $(r, s), r \in N_{l}, s \in N_{l}$.

LEMMA 4.6. There is an analytic function $r(s)$ from $N_{I^{\circ}}$ to $N_{I}$ such that

$$
a(r(s), s)=\min _{N_{t}} a(r, s) .
$$

Moreover, $a(r, s)>a(r(s), s)$ for $r \neq r(s)$.

Proof. Since $f(r)=a(r, s)$ is a continuous function on a finite-dimensional torus, it assumes its minimum at a point we call $r(s)$. Then, using the formula from theorem 2.4 with $(q, B)=(q(s), B(s))=\Phi^{-1}(r(s), s)$, the values of $a(r, s), r \in N_{l}$, are given by

$$
a=a(r(s), s)-\sum_{j \in I} b^{2}\left(\sigma_{j}-\mu_{j}\right) \prod_{\substack{i \in I \\ j \neq i}}\left(\frac{\sigma_{j}-\mu_{i}}{\sigma_{j}-\mu_{i}}\right)\left[\frac{y_{2}+b \xi_{*}}{a y_{2}+b y_{2}^{\prime}}\right]\left(1, \sigma_{j}\right),
$$

where $\left(\sigma_{j}, \xi_{*}\left(\sigma_{j}\right)\right)$ ranges over $C_{j}=\left\{(\mu, \xi)=\xi^{2}-\Delta(\mu)+1=0, \lambda_{2 j-1} \leq \mu \leq \lambda_{2 j}\right\}$ for $j \in I$, and we use remark 2.5 when $\sigma_{j}=\mu_{j}$. Since $y_{2}+b \xi$ must be non-positive on $C_{j}$, we see from lemma 4.3 that $\nu_{j}(q(s), B(s))=\mu_{j}(q(s), B(s)), j \in I$. Moreover, since 
lemma 4.3 also implies that

$$
\left(\mu_{j}(q(s), B(s)),-b^{-1} y_{2}\left(1, \mu_{j}(q(s), B(s)), q(s)\right)\right)
$$

is the only zero of $y_{2}+b \xi$ on $C_{j}$, it follows from (4.3) that $a(r(s), s)<a(r, s)$ for $r \neq r(s)$.

To see that $r(s)$ is real analytic, we first note that given $s_{0} \in N_{1^{\mathrm{c}}}, \lambda_{2 j-1}<$ $\mu_{j}\left(q\left(s_{0}\right), B\left(s_{0}\right)\right)<\lambda_{2 j}, j \in I$, and hence $\tilde{\mu}_{j}, j \in I$, defined in (1.13) are admissible coordinates on $N_{I}$ near $r\left(s_{0}\right)$. From (4.3)

$$
\frac{\partial^{2} a}{\partial \tilde{\mu}_{i} \partial \tilde{\mu}_{j}}\left(r\left(s_{0}\right), s_{0}\right)=\left\{\begin{array}{l}
0, \quad i \neq j, \\
-b^{2}\left[\frac{\left(\partial^{2} / \partial \mu^{2}\right)\left(y_{2}+b \xi_{*}\right)}{a \partial y_{2} / \partial \mu+b \partial y_{2}^{\prime} / \partial \mu}\right]\left(1, \mu_{j}\left(q\left(s_{0}\right), B\left(s_{0}\right)\right)\right), \quad i=j .
\end{array}\right.
$$

Moreover, since the zeros of $y_{2}+b \xi_{*}$ at

$$
\left(\mu_{j}\left(q\left(s_{0}\right), B\left(s_{0}\right)\right),-b^{-1} y_{2}\left(1, \mu_{i}\left(q\left(s_{0}\right), B\left(s_{0}\right)\right), q\left(s_{0}\right)\right)\right), \quad j \in I,
$$

are exactly of order two, $\left(\partial^{2} a / \partial \tilde{\mu}_{j}^{2}\right)\left(r\left(s_{0}\right), s_{0}\right)>0, j \in I$. Thus we may use the implicit function theorem to solve

$$
\frac{\partial a}{\partial \tilde{\mu}_{j}}(r, s)=0, \quad j \in I,
$$

with base point $\left(r\left(s_{0}\right), s_{0}\right)$. This yields an analytic function $\tilde{r}(s)$ defined near $s_{0}$ such that $\left(\partial a / \partial \tilde{\mu}_{j}\right)(\tilde{r}(s), s)=0, j \in I$, and $\left(\partial^{2} a / \partial \tilde{\mu}_{j} \partial \tilde{\mu_{i}}\right)(\tilde{r}(s), s)$ is positive definite. Setting $(\tilde{q}(s), \tilde{B}(s))=\Phi^{-1}(\tilde{r}(s), s)$, it follows from lemma $4.2(\mathrm{iv})$ that $\nu_{j}(\tilde{q}(s), \tilde{B}(s))=$ $\mu_{j}(\tilde{q}(s), \tilde{B}(s)), j \in I$, and hence $F_{j}(\mu, \xi)$ is of one sign on $C_{j}, j \in I$. The positivity of the Hessian of $a$ at $(F(s), s)$ then implies $\tilde{r}_{j}$ is non-positive. Thus (4.3) with $(r(s), s)$ replaced by $(\tilde{r}(s), s)$ implies $a(\tilde{r}(s), s) \leq a(r, s), r \in N_{I}$, and we conclude $\tilde{r}(s)=r(s)$ for $s$ near $s_{0}$.

The uniqueness statement in theorem 4.5 is a consequence of the following:

Proposition 4.7. Suppose $\left(q_{0}, B_{0}\right)=\Phi^{-1}\left(r_{0}, s_{0}\right)$ a fixed point for the *-involution of index I. Then $r_{0}=r\left(s_{0}\right)$ and

$$
a\left(r\left(s_{0}\right), s_{0}\right)>\min \{a(r(s), s), d(r(s), s)\}
$$

for $s \neq s_{0}$.

Proof. If $\left(q_{0}, B_{0}\right)$ is of index $I$, it follows immediately from lemma 4.6 that $r_{0}=r\left(s_{0}\right)$.

Let $a^{\prime}=a\left(r\left(s^{\prime}\right), s^{\prime}\right), d^{\prime}=d\left(r\left(s^{\prime}\right), s^{\prime}\right)$ and $a_{0}=a\left(r\left(s_{0}\right), s_{0}\right)=d\left(r\left(s_{0}\right), s_{0}\right)$, and suppose $\min \left\{a^{\prime}, d^{\prime}\right\} \geq a_{0}$. Since the $*$-involution fixes $y_{2}(1, \mu)$ and $\Delta(\mu)$, and hence $a\left(r(s)^{*}, s^{*}\right)=\min _{N_{l}} a\left(r, s^{*}\right)$, the involution must take $(r(s), s)$ to $\left(r\left(s^{*}\right), s^{*}\right)$ for some $s^{*} \in N_{r^{*}}$ by lemma 4.6. Hence we can assume without loss of generality that $a^{\prime} \leq d^{\prime}$. If $a^{\prime}>a_{0}$, then, as in the proof of lemma 3.2, we can use the flow of $V_{j}$ for $j$ sufficiently large, and hence in $I^{c}$ to move $a^{\prime}$ down below $a_{0}$. Thus we have $s^{\prime \prime}$ such that $a\left(r\left(s^{\prime}\right), s^{\prime \prime}\right)<a_{0}$ and hence $a\left(r\left(s^{\prime \prime}\right), s^{\prime \prime}\right)<a_{0}$, but $d\left(r\left(s^{\prime \prime}\right), s^{\prime \prime}\right)=d^{\prime}$. Since $N_{I^{c}} \cap\left\{d=d^{\prime}\right\}$ is connected and $a$ is continuous, it follows that there is an $s^{\prime \prime \prime}$ such that $a\left(r\left(s^{\prime \prime \prime}\right), s^{\prime \prime \prime}\right)=a_{0}$ and $d\left(r\left(s^{\prime \prime \prime}\right), s^{\prime \prime \prime}\right)=d^{\prime}$. Finally, using the involution, we get $s_{1}$ such that $a\left(r\left(s_{1}\right), s_{1}\right)=d^{\prime} \geq a_{0}$ and $d\left(r\left(s_{1}\right), s_{1}\right)=a_{0}=d\left(r\left(s_{0}\right), s_{0}\right)$. 
Let $\left(q^{\prime}, B^{\prime}\right)=\Phi^{-1}\left(r\left(s_{0}\right), s_{1}\right)$ and set

$$
\left(\tilde{\mu}_{j}, \xi_{*}\left(\tilde{\mu}_{j}\right)\right)=\left(\mu_{j}\left(q_{1}, B_{1}\right),-b^{-1} y_{2}\left(1, \mu_{j}\left(q_{1}, B_{1}\right), q_{1}\right)\right)
$$

for $j \in I^{c}$. By theorem 2.4 and the continuity of $\Phi^{-1}$

$$
a\left(r\left(s_{0}\right), s_{1}\right)=\lim _{N \rightarrow \infty} a_{0}-b^{2} \sum_{j \in S_{N}}\left(\tilde{\mu}_{j}-\mu_{j}\right) \prod_{\substack{i \in S_{N} \\ i \neq j}}\left(\frac{\tilde{\mu}_{j}-\mu_{i}}{\tilde{\mu}_{j}-\tilde{\mu}_{i}}\right)\left[\frac{y_{2}+b \xi_{*}}{a_{0} y_{2}+b y_{2}^{\prime}}\right]\left(1, \tilde{\mu}_{i}\right),
$$

where the $\mu_{i}$ and $y_{2}$ are computed at $\left(q_{0}, B_{0}\right)$ and $S_{N}=I^{c} \cap\{j \leq N\}$. Thus $a\left(r\left(s_{0}\right), s_{1}\right) \leq a_{0}$ and $d^{\prime}=a\left(r\left(s_{1}\right), s_{1}\right) \leq a\left(r\left(s_{0}\right), s_{1}\right)$. Since $d^{\prime} \geq a_{0}$, we conclude $d^{\prime}=$ $a_{0}$. This means that we can repeat the preceding argument with $\left(r\left(s^{\prime}\right), s^{\prime}\right)$ in place of $\left(r\left(s_{1}\right), s_{1}\right)$, i.e. (4.4) holds with $a\left(r\left(s_{0}\right), s_{1}\right)$ replaced by $a\left(r\left(s_{0}\right), s^{\prime}\right)$ and $\left(\tilde{\mu}_{j}, \xi_{*}\left(\tilde{\mu}_{j}\right)\right)$, $j \in I^{c}$, defined as before with $s_{1}$ replaced by $s^{\prime}$. Thus, since $a^{\prime} \geq a_{0}$ by assumption, we conclude $a^{\prime}=a_{0}$ and $\left(\tilde{\mu}_{j}, \xi_{*}\left(\tilde{\mu}_{j}\right)\right)=\left(\mu_{j}\left(q_{0}, B_{0}\right),-b^{-1} y_{2}\left(1, \mu_{j}\left(q_{0}, B_{0}\right), q_{0}\right)\right)$ for $j \in$ $I^{c}$. Since $d^{\prime}=d\left(r\left(s^{\prime}\right), s^{\prime}\right)=a_{0}=d\left(r\left(s_{0}\right), s_{0}\right)$, we have $s^{\prime}=s_{0}$.

Proposition 4.7 implies that to find a fixed point of the involution with index $\mathbf{I}$, we only need find an $s_{0} \in I$, where $\sup _{N_{r}} a(r(s), s)$ is assumed. However, since $N_{I^{c}}$ is not compact, we have not been able to do this directly. Instead we will exploit a connection between maxima of $a$ and minima of

$$
\int_{0}^{1}(k+q)^{2} d x
$$

on $M \cap\{a=d\}$.

LEMMA 4.8. For any real constant $k$, the derivative of $\frac{1}{4} \int_{0}^{1}(k+q)^{2} d x$ on the flow of the vector field $(\dot{q}, \dot{B})=V(q, B, \lambda)$ in theorem 2.1 is given by

$$
\left[\frac{1}{4} \int_{0}^{1}(k+q)^{2} d x\right]=\left[(d-a)\left((\lambda+k) y_{2}+y_{1}\right)+(c+(\lambda+k) b)\left(y_{1}-y_{2}^{\prime}\right)\right](1, \lambda, q) .
$$

Proof. We have

$$
\left[\frac{1}{4} \int_{0}^{1}(k+q)^{2} d x\right]=\frac{1}{2} \int_{0}^{1}(k+q) \dot{q} d x .
$$

Since $(2 / b)(a+b)+\int_{0}^{1} q d x$ is constant on $M$ (see (3.2)) and the vector field is tangent to $M$, we have (see (2.7))

$$
\frac{k}{2} \int_{0}^{1} \dot{q} d x=-\frac{k}{b}(\dot{a}+\dot{d})=\left[k(d-a) y_{2}+k b\left(y_{1}-y_{2}^{\prime}\right)\right](1, \lambda) .
$$

Using the abbreviations

$$
\langle y\rangle=a y(1, \lambda)+b y^{\prime}(1, \lambda), \quad[y]=c y(1, \lambda)+d y^{\prime}(1, \lambda),
$$

the formula for $\partial \Delta(\lambda) / \partial q(x)$ given in (2.3) becomes

$$
\frac{\partial \Delta(\lambda)}{\partial q(x)}=y_{1}(x)\left(\left\langle y_{2}\right\rangle y_{1}(x)-\left\langle y_{1}\right\rangle y_{2}(x)\right)+y_{2}(x)\left(\left[y_{2}\right] y_{1}(x)-\left[y_{1}\right] y_{2}(x)\right) \text {. }
$$

Substituting the derivative of this expression for $\frac{1}{2} \dot{q}$ and systematically replacing $q y$ 
by $y^{\prime \prime}+\lambda y$, we arrive at

$$
\begin{aligned}
\frac{1}{2} \int_{0}^{1} q \dot{q} d x= & \left.\left\langle y_{2}\right\rangle\left(\left(y_{1}^{\prime}\right)^{2}+\lambda y_{1}^{2}\right)\right|_{0} ^{1}-\left.\left\langle y_{1}\right\rangle\left(y_{1}^{\prime} y_{2}^{\prime}+\lambda y_{1} y_{2}\right)\right|_{0} ^{1} \\
& +\left.\left[y_{2}\right]\left(y_{1}^{\prime} y_{2}^{\prime}+\lambda y_{1} y_{2}\right)\right|_{0} ^{1}-\left.\left[y_{1}\right]\left(\left(y_{2}^{\prime}\right)^{2}+\lambda y_{2}^{2}\right)\right|_{0} ^{1} \\
= & {\left[\left(a y_{2}+b y_{2}^{\prime}\right)\left(\left(y_{1}^{\prime}\right)^{2}+\lambda y_{1}^{2}\right)+\left(c y_{2}+d y_{2}^{\prime}-a y_{1}-b y_{1}^{\prime}\right)\left(y_{1}^{\prime} y_{2}^{\prime}+\lambda y_{1} y_{2}\right)\right.} \\
& \left.-\left(c y_{1}+d y_{1}^{\prime}\right)\left(\left(y_{2}^{\prime}\right)^{2}+\lambda y_{2}^{2}\right)\right](1, \lambda)-\left[\lambda\left(a y_{2}+b y_{2}^{\prime}\right)-c y_{1}-d y_{1}^{\prime}\right](1, \lambda) \\
= & {\left[-a y_{1}^{\prime}+\lambda b y_{1}-c y_{2}^{\prime}+\lambda d y_{2}-\lambda a y_{2}-\lambda b y_{2}^{\prime}+c y_{1}+d y_{1}^{\prime}\right](1, \lambda) } \\
= & {\left[(d-a)\left(y_{1}^{\prime}+\lambda y_{2}\right)+(c+\lambda b)\left(y_{1}-y_{2}^{\prime}\right)\right](1, \lambda) . }
\end{aligned}
$$

In passing to the third equality, we used $\left(y_{1} y_{2}^{\prime}-y_{2} y_{1}^{\prime}\right)=1$ four times. Combining (4.6) and (4.7) gives (4.5).

The following proposition completes the proof of theorem 4.5 .

Proposition 4.9. Given a finite index set $\mathbf{I}$, let $M_{I}=\Phi^{-1}\left(\left\{(r(s), s): s \in N_{I^{c}}\right\}\right)$, where $r(s)$ is the analytic function of lemma 4.6. Then for $k$ sufficiently large

$$
\lim _{M_{1} \cap\{a=d\}} \int_{0}^{1}(q+k)^{2} d x
$$

is assumed at a fixed point of index $\mathbf{I}$.

Proof. First we show the minimum is assumed. Since, as noted in the proof of proposition 4.7, the involution leaves $M_{I}$ invariant if $a-d$ is not identically zero on $M_{I}$, it must be of two signs on $M_{I}$. Hence, since $N_{I}$ is connected, we conclude $M_{l} \cap\{a=d\}$ is non-empty.

Choosing a sequence $\left(q_{j}, B_{j}\right)$ in $M_{1} \cap\{a=d\}$ on which

$$
\frac{1}{4} \int_{0}^{1}(q+k)^{2} d x
$$

tends to its infimum, we can choose a subsequence such that $q_{j_{i}} \rightarrow q_{\infty}$ weakly in $L^{2}[0,1]$. The sequence $a_{j}=d_{j}$ is bounded, since $(2 / b)\left(a_{j}+d_{j}\right)+\int_{0}^{1} q_{j} d x$ is constant on $M$, and $c_{j}=a_{j}^{2}-1 / b$, since $B_{j} \in \operatorname{SL}(2, \mathbf{R})$. Thus we may assume $B_{j_{i}} \rightarrow B_{\infty}$ with $a_{\infty}=d_{\infty}$.

To see that $\left(q_{\infty}, B_{\infty}\right) \in M_{I} \cap\{a=d\}$, we note first that theorem 1.5 of [6] implies, for $l=1,2, y_{l}\left(x, \lambda, q_{j_{i}}\right) \rightarrow y_{l}\left(x, \lambda, q_{\infty}\right)$ uniformly on bounded subsets of $[0,1] \times \mathbf{C}$. From the formulae

$$
\begin{gathered}
y_{1}^{\prime}(x, \lambda, q)=-\lambda^{1 / 2} \sin \lambda^{1 / 2} x+\int_{0}^{x} \cos \lambda^{1 / 2}(x-t) q(t) y_{1}(t, \lambda, q) d t \\
y_{2}^{\prime}(x, \lambda, q)=\cos \lambda^{1 / 2} x+\int_{0}^{x} \cos \lambda^{1 / 2}(x-t) q(t) y_{2}(t, \lambda, q) d t
\end{gathered}
$$

one sees that $y_{l}^{\prime}\left(1, \lambda, q_{j_{i}}\right) \rightarrow y_{l}^{\prime}\left(1, \lambda, q_{\infty}\right)$ for $\lambda \in C$. Thus $\Delta\left(\lambda, q_{\infty}, B_{\infty}\right)=\Delta(\lambda)$ and $\left(q_{\infty}, B_{\infty}\right) \in M$. Finally, since

$$
y_{2}\left(1, \mu, q_{j_{i}}\right)+b \xi_{*}(\mu) \rightarrow y_{2}\left(1, \mu, q_{\infty}\right)+b \xi_{*}(\mu)
$$

on $C_{j}$ for $j \in I$, the characterization of $r(s)$ in lemma 4.6 shows $\left(q_{\infty}, B_{\infty}\right) \in M_{l}$. 
Since $\int_{0}^{1}(q+k)^{2} d x$ can only jump down on weakly convergent sequences,

$$
\int_{0}^{1}\left(k+q_{\infty}\right)^{2} d x=\min _{M_{1} \cap\{a=d\}} \int_{0}^{1}(k+q)^{2} d x
$$

and the derivative of

$$
\int_{0}^{1}\left(k+q_{\infty}\right)^{2} d x
$$

along any differentiable curve in $M_{I} \cap\{a=d\}$ must vanish when the curve passes through $\left(q_{\infty}, B_{\infty}\right)$. If $M_{I} \cap\{a=d\}$ is a smooth submanifold of $M$, the preceding condition is equivalent to: the derivative of

$$
\int_{0}^{1}\left(k+q_{\infty}\right)^{2} d x
$$

along any differentiable curve in $M$, tangent to $M_{I}$ and $\{a=d\}$ at $\left(q_{\infty}, B_{\infty}\right)$, vanishes when the curve passes through $\left(q_{\infty}, B_{\infty}\right)$. The second form is much more convenient for us, so we will show that $\{a=d\}$ and $M_{I}$ intersect transversally and hence $M_{l} \cap\{a=d\}$ is smooth.

Given $(q, B) \in M_{I} \cap\{a=d\}$, theorems 1.7 and 2.2 imply that $\left\{V_{n_{j}}(q, B): j \in I\right\}$ is a basis for the tangent space at $(q, B)$ to $\Phi^{-1}\left(N_{I} \times\{s\}\right)$, where $s$ is the component of $\Phi(q, B)$ in $N_{I^{c}}$. Thus, since $V_{\lambda}(q, B)$ is in the tangent space to $M$ at $(q, B)$, there are unique $c_{j} \in \mathbf{R}$ such that $V_{\lambda}-\sum_{j \in I} c_{j} V_{n_{j}}$ is in the tangent space to $M_{I}$ at $(q, B)$. Let $\gamma(t)$ be a smooth curve in $M$ with $\gamma(0)=(q, B)$ and $\gamma^{\prime}(0)=V_{\lambda}-\sum_{j \in I} c_{j} V_{\mu_{j}}$. Then, since $\mu_{j}(q, B)=\nu_{j}(q, B), j \in I$, for $(q, B) \in M_{I}$,

$$
\left.\frac{d}{d t}[d-a](\gamma(t))\right|_{t=0}=\left[b(a+d) y_{2}+b^{2}\left(y_{2}^{\prime}+y_{1}\right)\right](1, \lambda, q) \equiv h(\lambda)
$$

and, since $h(\lambda)=2 b^{2} \cos \lambda^{1 / 2}+O\left(\lambda^{-1 / 2}\right)$, it follows that for suitable $\lambda$

$$
\frac{d}{d t}[d-a](\gamma(t)) \neq 0 .
$$

Thus $\{d=a\}$ and $M_{I}$ intersect transversally at $(q, B)$.

Now continuing with the same notation, let $(q, B)=\left(q_{\infty}, B_{\infty}\right)$ and let $\gamma(t)$ be a smooth curve in $M$ with $\gamma(0)=\left(q_{\infty}, B_{\infty}\right)$ and, for $l \in I^{c}$,

$$
\gamma^{\prime}(0)=\left[V_{\mu_{l}}-\frac{h\left(\mu_{l}\right)}{h(\lambda)}\left(V_{\lambda}-\sum_{j \in I} c_{j} V_{\mu_{j}}\right)\right]\left(q_{\infty}, B_{\infty}\right) \text {. }
$$

By construction $\gamma^{\prime}(0)$ is tangent to $M_{I}$ and $\{a=d\}$, hence

$$
\left.\frac{d}{d t}\left(\frac{1}{4} \int_{0}^{1}(k+q(\gamma(t)))^{2} d x\right)\right|_{t=0}=0
$$

Since $d_{\infty}=a_{\infty}$, and $\nu_{j}\left(q_{\infty}, B_{\infty}\right)=\mu_{j}\left(q_{\infty}, B_{\infty}\right)$ for $j \in I$, it follows that $y_{2}^{\prime}\left(1, \mu_{j}, q_{\infty}\right)=$ $y_{1}\left(1, \mu_{j}, q_{\infty}\right)$ for $j \in I$. Thus, when one uses lemma 4.8 to compute the derivative in (4.8), it yields

$$
0=\left(c_{\infty}+b\left(\mu_{l}+k\right)\right)\left[y_{1}-y_{2}^{\prime}\right]\left(1, \mu_{l}, q_{\infty}\right)-\left(c_{\infty}+b(\lambda+k)\right)\left[y_{1}-y_{2}^{\prime}\right]\left(1, \lambda, q_{\infty}\right) \frac{h\left(\mu_{l}\right)}{h(\lambda)} .
$$


However, since $h\left(\mu_{t}\right)=\left[b^{2}\left(y_{1}-y_{2}^{\prime}\right)\right]\left(1, \mu_{l}, q_{\infty}\right)$, we have

$$
0=\left(\left[y_{1}-y_{2}^{\prime}\right]\left(1, \mu_{l}, q_{\infty}\right)\right)\left(\left(c_{\infty}+b\left(\mu_{l}+k\right)\right)-b^{2} \frac{\left(c_{\infty}+b(\lambda+k)\right)}{h(\lambda)}\left[y_{1}-y_{2}^{\prime}\right]\left(1, \lambda, q_{\infty}\right)\right) \text {. }
$$

Thus either $\left[y_{1}-y_{2}^{\prime}\right]\left(1, \mu_{l}, q_{\infty}\right)=0$ or the other factor in (4.9) vanishes identically in $\lambda$. Since $c>-1 / b$ on $M \cap\{a=d\}$, we may assume that $k$ was chosen large enough at the outset that $c_{\infty}+b(\lambda+k)$ is strictly positive on all the gaps. Thus, if the second factor vanishes identically, $\left[y_{1}-y_{2}^{\prime}\right]\left(1, \lambda, q_{\infty}\right)$ must vanish at every zero of $h(\lambda)$. Note that

$$
h(\lambda)=b\left(\left[a_{\infty} y_{2}+b y_{2}^{\prime}\right]\left(1, \lambda, q_{\infty}\right)+\left[d_{\infty} y_{2}+b y_{1}\right]\left(1, \lambda, q_{\infty}\right)\right) \equiv b f(\lambda)+b f^{*}(\lambda) .
$$

Since $f$ and $f^{*}$ have the same sign at $\lambda_{2 n-1}$ and the same but opposite sign at $\lambda_{2 n}$ for all $n$, we see that $h(\lambda)$ has a zero $\xi_{n}$ in $\lambda_{2 n-1} \leq \lambda \leq \lambda_{2 n}$ for all $n$. If $\left[y_{1}-y_{2}^{\prime}\right]\left(1, \xi_{n}\right)=0$ as well, then $\xi_{n}=\mu_{n}\left(q_{\infty}, B_{\infty}\right)=\nu_{n}\left(q_{\infty}, B_{\infty}\right)$. Thus, if the second factor in (4.9) vanishes identically, we can still conclude $\left[y_{1}-y_{2}^{\prime}\right]\left(1, \mu_{l}, q_{\infty}\right)=0$. Since $l$ was an arbitrary element in $I^{c}$, we get $\nu_{j}\left(q_{\infty}, B_{\infty}\right)=\mu_{j}\left(q_{\infty}, B_{\infty}\right)$ for all $j$, and by lemma 4.4 $\left(q_{\infty}, B_{\infty}\right)$ is a fixed point of the $*$-involution.

Now we only need show that $\left(q_{\infty}, B_{\infty}\right)$ has index $I$. As in the preceding paragraph, we assume $k$ was chosen so that $c_{\infty}+b(\lambda+k)$ is positive on all gaps. Since $h\left(\mu_{j}, q_{\infty}, B_{\infty}\right)=0$ for all $j$, given $l \in I^{c}, V_{\mu_{l}}\left(q_{\infty}, B_{\infty}\right)$ is tangent to $M \cap\{a=d\}$. However, $V_{\mu_{1}}$ is tangent to $M_{I}$ as well. To see this, note that, as in the proof of lemma 4.6, near $\Phi\left(q_{\infty}, B_{\infty}\right), d, r$ and the $\tilde{\mu}$ are admissible coordinates on $N \cap$ $\left\{d<d_{\infty}\right\}$. As in the proof of lemma 4.6,

$$
\frac{\partial^{2} a}{\partial \tilde{\mu}_{i} \partial \tilde{\mu}_{j}}\left(\Phi\left(q_{\infty}, B_{\infty}\right)\right)=0, \quad i \neq j .
$$

Thus, differentiating the equations

$$
\frac{\partial a}{\partial \tilde{\mu}_{j}}(r(s), s)=0, \quad j \in I,
$$

with respect to $\tilde{\mu}_{l}$ we see

$$
\left.\frac{\partial \tilde{\mu}_{j}(r(s), s)}{\partial \tilde{\mu}_{i}}\right|_{(r(s), s)=\Phi\left(q_{\infty}, B_{\infty}\right)}=0, \quad j \in I .
$$

Thus $V_{\mu_{1}}\left(q_{\infty}, B_{\infty}\right)$ is tangent to $M_{I}$ at $\left(q_{\infty}, B_{\infty}\right)$.

Note that the image of $\left\{V_{\mu_{j}}(q, B): j=1,2, \ldots\right\}$ under $\Phi^{\prime}(q, B)$ is clearly codimension 1 in the tangent space to $N \cap\left\{d<d_{\infty}\right\}$ at $\Phi(q, B)$. Moreover, $V_{\lambda}(q, B)$ for $\lambda \neq \mu_{j}(q, B), j=1,2, \ldots$, is not in the closed linear span of

$$
\left\{V_{\mu_{j}}(q, B): j=1,2, \ldots\right\}
$$

since $\dot{d} \neq 0$ on its integral curve at $(q, B)$. Thus, since $\Phi^{\prime}$ is an isomorphism, we may conclude that the closed linear span of $\left\{V_{\lambda}(q, B): \lambda \in \mathbf{R}\right\}$ is the tangent space to $M$ at $(q, B)$. 
If we set $(\dot{q}, \dot{B})=V_{\lambda}\left(q_{\infty}, B_{\infty}, \lambda\right)$, it follows immediately that $\dot{a}+\dot{d}=0$ for all $\lambda$. Likewise by lemma 4.8

$$
\frac{1}{4} \int_{0}^{1}(k+q)^{2} d x=D
$$

for all $\lambda$. Thus the gradients of $a+d$ and $\int_{0}^{1}(k+q)^{2} d x$ as functions on $M$ vanish at $\left(q_{\infty}, B_{\infty}\right)$. Hence by the chain rule, given $\gamma(t)$ in $M$ with $\gamma(0)=\left(q_{\infty}, B_{\infty}\right)$, the second derivatives at $t=0$ of these functions restricted to $\gamma(t)$ depend only on $\gamma^{\prime}(0)$.

To use the observation of the preceding paragraph, we note that the second derivative at $t=0$ of $\frac{1}{4} \int_{0}^{1}(k+q)^{2} d x$ along $\gamma(t)$ in $M_{I} \cap\{a=d\}$ with $\gamma(0)=\left(q_{\infty}, B_{\infty}\right)$ and $\gamma^{\prime}(0)=V_{\mu_{i}}\left(q_{\infty}, B_{\infty}\right)$ must be non-negative. Hence the same is true if we take $V(t)$ to be the integral curve through $\left(q_{\infty}, B_{\infty}\right)$ of $V_{\xi_{l}(q, B)}(q, B)$, where $\xi_{l}(q, B)$ is the zero of $h(\lambda, q, B)$ in $\lambda_{2 l-1} \leq \mu \leq \lambda_{21}$. An argument from Rouche's theorem like that used in the proof of theorem 1.3 shows $h(\lambda, q, B)$ has exactly one root counted by multiplicity in $\lambda_{2 l-1} \leq \mu \leq \lambda_{2 l}$ for each $l$. Thus $\xi_{l}(q, B)$ is a well defined analytic function on $M$. Since $\dot{a}-\dot{d}=0$ on $\gamma, \gamma(t)$ lies in $M \cap\{a=d\}$.

By theorem 4.8 for $t$ near zero

$$
\frac{d}{d t}\left(\int_{0}^{1}(k+q(\gamma(t)))^{2} d x\right)=\left(c(t)+b\left(\xi_{l}(t)+k\right)\right)\left[y_{1}-y_{2}^{\prime}\right]\left(1, \xi_{l}(t), q(t)\right)
$$

and by (2.7)

$$
\frac{d}{d t}[a+d](\gamma(t))=-b^{2}\left[y_{1}-y_{2}^{\prime}\right]\left(1, \xi_{l}(t), q(t)\right)
$$

Thus we must have $\left.\left(d^{2} / d t^{2}\right)([a+d](\gamma(t)))\right|_{t=0} \leq 0$. Again, since the gradient of $a+d$ on $M$ vanishes at $\left(q_{\infty}, B_{\infty}\right)$, this remains true if we replace $\gamma(t)$ by the integral curve of $V_{\mu}$ through $\left(q_{\infty}, B_{\infty}\right)$. Thus we conclude $F_{l}\left(\mu, \xi, q_{\infty}, B_{\infty}\right)$ is non-negative on $C_{l}$, $l \in I^{c}$, and $\left(q_{\infty}, B_{\infty}\right)$ has index $\mathbf{I}$.

In the course of the proof of proposition 4.9 we saw that a fixed point of the involution was necessarily a critical point of $a+d$ on $M$. Conversely, lemma 4.5 implies a critical point of $a+d$ must be fixed by the involution. Thus we have:

Corollary 4.10. The fixed points of the involution on $M$ coincide with the critical points of $a+d$ on $M$.

\section{The range of $B$}

If we consider $B$ as a function on $M$, then its range is simply the set of boundary conditions which give rise to the generalized periodic spectrum defining $M$ for some potential $q$. Since $b$ is constant on and $c=b^{-1}(a d-1)$, it suffices to determine the range of $(a, d)$. Using the results of $\S \S 2$ and 4 , one can find the range of $(a, d)$ on $M_{I}$ for all $\mathbf{I}$.

Theorem 5.1. Let $\left(q_{l}, B_{l}\right)$ be the fixed point of * on $M$ of index I. Then the range of $(a, d)$ on $M_{l}$ is $\left.\left\{(a, d): a+d<2 a_{l}\right\} \cup\left(a_{l}, a_{l}\right)\right\}$. In particular, this gives the range of $B$ on $M$ when $I$ is the empty set. 
In proving theorem 5.1, we will use one of the formulae of theorem 2.4 extended to the case where one moves all the $\mu$, namely

$$
\hat{a}=a-b^{2} \sum_{j=1}^{\infty}\left(\sigma_{j}-\mu_{j}\right)\left(\prod_{i \pm j} \frac{\sigma_{j}-\mu_{i}}{\sigma_{j}-\sigma_{i}}\right)\left[\frac{y_{2}+b \xi_{*}}{a y_{2}+b y_{2}^{\prime}}\right]\left(1, \sigma_{j}, q\right) \text {. }
$$

We claim that $(5.1)$ is valid whenever

$$
s=\left(b, d, \sigma_{1},-b \xi_{*}\left(\sigma_{1}\right), \sigma_{2},-b \xi_{*}\left(\sigma_{2}\right), \ldots\right)
$$

is in the range of $\Phi$. From the continuity of $\Phi^{-1}$, defining $(\hat{q}, \hat{B})=\Phi^{-1}(s)$, it follows that $\hat{a}=\lim _{N \rightarrow \infty} a_{N}$, where

$$
a_{N}=a-b^{2} \sum_{j=1}^{N}\left(\sigma_{k}-\mu_{k}\right)\left(\prod_{\substack{i=1 \\ i \neq j}}^{N} \frac{\sigma_{j}-\mu_{i}}{\sigma_{j}-\sigma_{i}}\right)\left[\frac{y_{2}+b \xi_{*}}{a y_{2}+b y_{2}^{\prime}}\right]\left(1, \sigma_{j}, q\right) .
$$

Thus (5.1) will hold if the sum of products on the right-hand side of (5.2) converges to the right-hand side of (5.1). This holds under the weaker assumption $\left|\sigma_{k}-\mu_{k}\right|<C$ for all $k$ and $\left|\xi_{*}\left(\sigma_{k}\right)\right|<1$ for $k>k_{0}$. To see this, we note that

and

$$
\prod_{\substack{i=1 \\ i \neq j}}^{N} \frac{\sigma_{j}-\mu_{i}}{\sigma_{j}-\sigma_{i}}=\prod_{i=1}^{N}\left(1+\frac{\sigma_{i}-\mu_{i}}{\sigma_{j}-\sigma_{i}}\right)
$$

$$
\left|\frac{\sigma_{i}-\mu_{i}}{\sigma_{j}-\sigma_{i}}\right| \leq \frac{C}{\pi^{2}\left|\left(j-\frac{1}{2}\right)^{2}-\left(i-\frac{1}{2}\right)^{2}\right|-C^{\prime}}
$$

for some $C^{\prime}$ independent of $i, j$. Thus we see that the products are convergent and uniformly bounded in $j$. Using theoreem 1.1 and lemma 4.2(iv), we estimate the term $\left(a y_{2}+b \xi_{*}\right)\left(a y_{2}+b y_{2}^{\prime}\right)^{-1}$ by

$$
\begin{aligned}
\left|\left[\frac{y_{2}+b \xi_{*}}{a y_{2}+b y_{2}^{\prime}}\right](1, \mu)\right| & =\left|\left[\frac{d y_{2}+b y_{1}}{y_{2}+b \xi_{*}^{-1}}\right](1, \mu)\right| \\
& =\frac{b \cos \mu^{1 / 2}+O\left(\mu^{-1 / 2}\right)}{(b / 2) \Delta(\mu)\left(1+\sqrt{1-4 \Delta(\mu)^{-2}}\right)+O\left(\mu^{-1 / 2}\right)} \\
& =\frac{b \cos \mu^{1 / 2}+O\left(\mu^{-1 / 2}\right)}{-b \mu^{1 / 2} \sin \mu^{1 / 2}+O(1)} .
\end{aligned}
$$

Thus, since we have $\left(\sigma_{j}\right)^{1 / 2}=\pi\left(j-\frac{1}{2}\right)+O(1 / j)$,

$$
\left|\left[\frac{y_{2}+b \xi_{*}}{a y_{2}+b y_{2}^{\prime}}\right]\left(1, \sigma_{j}\right)\right| \leq \frac{K}{j^{2}} \text {. }
$$

Thus the right-hand side of (5.1) is convergent and, by dominated convergence, is the limit of the sequence of the $a_{N}$.

Since proposition 4.7 implies

$$
a_{I}=\max _{M_{1}}(\min \{a, d\}),
$$

if $(a, d)=\left(a_{1}, d_{1}\right)$ at $\left(q_{1}, B_{1}\right) \in M_{l}$, we may assume, using the involution if necessary, that $a_{1} \leq a_{1}$. Choosing $k$ sufficiently large, we may use the flow of $V_{k}$ as in the proof of lemma 3.2 to move $a_{I}$ down to $a_{1}$, moving $\left(q_{I}, B_{I}\right)$ to $\left(q_{2}, B_{2}\right)$. Then, applying the involution, we arrive at $\left(q_{2}^{*}, B_{2}^{*}\right)$ with $\left(a_{2}^{*}, a_{2}^{*}\right)=\left(a_{1}, a_{1}\right)$. Since $\left(q_{1}^{*}, B_{1}^{*}\right)$ has 
$\left(a_{1}^{*}, d_{1}^{*}\right)=\left(d_{1}, a_{1}\right)$, and we know from lemma 3.1 that $\Phi\left(M \cap\left\{d=a_{1}\right\}\right)=N \cap$ $\left\{d=a_{1}\right\}$, it follows that we can choose $\left(\sigma_{k}, \xi_{*}\left(\sigma_{k}\right)\right), k=1, \ldots$, so that

$$
d_{1}=a_{I}-b^{2} \sum_{j=1}^{\infty}\left(\sigma_{j}-\nu_{j}\right)\left(\prod_{i \neq j}^{\infty} \frac{\sigma_{j}-\nu_{i}}{\sigma_{j}-\sigma_{i}}\right)\left[\frac{y_{2}+b \xi_{*}}{a_{2}^{*} y_{2}+b y_{2}^{\prime}}\right]\left(1, \sigma_{j}, q_{2}^{*}\right)
$$

as in (5.1), where $\nu_{k}$ is the zero of $\left[a_{2}^{*}+b y_{2}^{\prime}\right]\left(1, \lambda, q_{2}^{*}\right)$ in $\lambda_{2 j-1} \leq \lambda \leq \lambda_{2 j}$.

To arrive at the formula we need for the proof of theorem 5.1, we need to follow the operations that lead to $\left(q_{2}^{*}, B_{2}^{*}\right)$ back to $\left(q_{I}, B_{I}\right)$. This is not difficult. We have $y_{2}\left(1, \lambda, q_{2}^{*}\right)=y_{2}\left(1, \lambda, q_{2}\right)$ and, combining (2.20) and (2.21),

$$
y_{2}\left(1, \lambda, q_{2}\right)=y_{2}\left(1, \lambda, q_{1}\right)+\frac{a_{1}-a_{I}}{b^{2}}\left[\frac{a_{I} y_{2}+b y_{2}^{\prime}}{\lambda-\mu_{k}}\right]\left(1, \lambda, q_{I}\right),
$$

where $\mu_{k}$ is the zero of $\left[a_{1} y_{2}+b y_{2}^{\prime}\right]\left(1, \lambda, q_{1}\right)$ in $\lambda_{2 k-1}<\lambda<\lambda_{2 k}$. Since $\left[a_{2}^{*} y_{2}+b y_{2}^{\prime}\right] \times$ $\left(1, \lambda, q_{2}^{*}\right)$ and $\left[a_{1} y_{2}+b y_{2}^{\prime}\right]\left(1, \lambda, q_{I}\right)$ are entire functions of order $\frac{1}{2}$ with the same asymptotics as $\lambda \rightarrow \infty$,

$$
\left[a_{2}^{*} y_{2}+b y_{2}^{\prime}\right]\left(1, \lambda, q_{2}^{*}\right)=\left(\prod_{i=1}^{\infty} \frac{\lambda-\nu_{i}}{\lambda-\mu_{i}}\right)\left[a_{I} y_{2}+b y_{2}^{\prime}\right]\left(1, \lambda, q_{I}\right) .
$$

Substituting (5.6) and (5.7) into (5.5), we have

$$
d_{1}=a_{I}-b^{2} \sum_{j=1}^{\infty}\left(\prod_{\substack{i=1 \\ j \neq i}}^{\infty} \frac{\sigma_{j}-\mu_{i}}{\sigma_{j}-\sigma_{i}}\right)\left(\left[\frac{y_{2}+b \xi_{*}}{a_{l} y_{2}+b y_{2}^{\prime}}\right]\left(1, \sigma_{j}, q_{I}\right)+\frac{a_{1}-a_{I}}{b^{2}} \frac{\sigma_{j}-\mu_{j}}{\sigma_{j}-\mu_{k}}\right) .
$$

Note that the infinite products in (5.8) are convergent by (5.3).

LEMMA 5.2

$$
\sum_{j=1}^{\infty}\left(\frac{\sigma_{j}-\mu_{j}}{\sigma_{j}-\mu_{k}}\right) \prod_{i \neq j} \frac{\sigma_{j}-\mu_{i}}{\sigma_{j}-\sigma_{i}}=1
$$

Proof. Since $\left|\sigma_{j}-\pi^{2}\left(j-\frac{1}{2}\right)^{2}\right|<C$ for all $j$,

$$
S_{N}=\sum_{j=1}^{N}\left(\frac{\sigma_{j}-\mu_{j}}{\sigma_{j}-\mu_{k}}\right) \prod_{i \neq j} \frac{\sigma_{j}-\mu_{i}}{\sigma_{j}-\sigma_{i}}
$$

is the sum of the residues of

$$
f(z)=\frac{1}{z-\mu_{k}} \prod_{i=1}^{\infty} \frac{z-\mu_{i}}{z-\sigma_{i}}
$$

in $|z|<\pi^{2} N^{2}$ for $N$ sufficiently large. On $|z|=\pi^{2} N^{2}$, estimating as in (5.3),

$$
\left|\frac{z-\mu_{i}}{z-\sigma_{i}}-1\right|=\left|\frac{\sigma_{i}-\mu_{i}}{z-\sigma_{i}}\right| \leq C\left(\left|\pi^{2} N^{2}-\pi^{2}\left(i-\frac{1}{2}\right)^{2}\right|-C\right)^{-1},
$$

where $C$ is independent of $N$ and $i$. Hence, as $N \rightarrow \infty, \prod_{i=1}^{\infty}\left(z-\mu_{i}\right)\left(z-\sigma_{i}\right)^{-1} \rightarrow 1$ uniformly on $|z|=\pi^{2} N^{2}$. Hence, as $|z|=\pi^{2} N^{2}$,

$$
\frac{1}{z-\mu_{k}} \prod_{i=1}^{\infty} \frac{z-\mu_{i}}{z-\sigma_{i}}=\frac{1+O(1)}{z-\mu_{k}} .
$$

Using $\oint_{|z|=\pi^{2} N^{2}} f(z) d z=2 \pi i S_{N}$, we conclude $\lim _{N \rightarrow \infty} S_{N}=1$. To see that $\lim _{N \rightarrow \infty} S_{N}$ is the sum in (5.9), one may again use (5.3). 
Substituting (5.9) into (5.8) gives a formula which implies theorem 5.1:

$$
a_{1}+d_{1}=2 a_{I}-b^{2} \sum_{j=1}^{\infty}\left(\sigma_{j}-\mu_{j}\right)\left(\prod_{i \neq j} \frac{\sigma_{j}-\mu_{i}}{\sigma_{j}-\sigma_{i}}\right)\left[\frac{y_{2}+b \xi_{*}}{a_{i} y_{2}+b y_{2}^{\prime}}\right]\left(1, \sigma_{j}, q_{1}\right) \text {. }
$$

Proof of theorem 5.1. Since $\left(q_{1}, B_{1}\right)$ is in $M_{I}$, and, as noted in the proof of proposition 4.7, $M_{I}$ is *-invariant, it follows that $\left(q_{1}^{*}, B_{1}^{*}\right) \in M_{I}$. Thus, if we move $\sigma_{j}$ to $\mu_{j}$ and $\xi_{*}\left(\sigma_{j}\right)$ to $\xi_{*}\left(\mu_{j}\right)$ for $j \in I$, we will not decrease $a_{1}^{*}$, and (5.5) will become

$$
d_{1} \leq a_{I}-b^{2} \sum_{j=1}^{\infty}\left(\tilde{\sigma}_{j}-\nu_{j}\right)\left(\prod_{i \neq j} \frac{\tilde{\sigma}_{j}-\nu_{i}}{\tilde{\sigma}_{j}-\tilde{\sigma}_{i}}\right)\left[\frac{y_{2}+b \xi_{*}}{a_{2}^{*} y_{2}+b y_{2}^{\prime}}\right]\left(1, \tilde{\sigma}_{j}, q_{2}^{*}\right),
$$

where

$$
\tilde{\sigma}_{j}= \begin{cases}\sigma_{j}, & j \in I^{c}, \\ \mu_{j}, & j \in I .\end{cases}
$$

Hence the remainder of the proof of (5.10) after (5.5) shows

$$
a_{1}+d_{1} \leq 2 a_{I}-b^{2} \sum_{j \in I^{c}}\left(\sigma_{j}-\mu_{j}\right)\left(\prod_{\substack{i \neq j \\ i \in I^{c}}} \frac{\sigma_{j}-\mu_{i}}{\sigma_{j}-\sigma_{i}}\right)\left[\frac{y_{2}+b \xi_{*}}{a_{1} y_{2}+b y_{2}^{\prime}}\right]\left(1, \sigma_{j}, q_{1}\right)
$$

Since $\left(q_{l}, B_{l}\right)$ has index $\mathrm{I}$,

$$
F_{j}(\mu, \xi)=\left(\mu-\mu_{j}\right)\left[\frac{y_{2}+b \xi}{a_{1} y_{2}+b y_{2}^{\prime}}\right]\left(1, \mu, q_{I}\right)
$$

is non-negative on $C_{j}$ for $j \in I^{c}$. Thus (5.11) shows $a_{1}+d_{1} \leq 2 a_{l}$. Moreover, if $a_{1}+d_{1}=2 a_{l}$, then $\sigma_{j}=\mu_{j}$ for $j \in I^{c}$. By theorems 1.3 and 1.4

$$
\begin{aligned}
\mu_{j} & =\pi^{2}\left(j-\frac{1}{2}\right)^{2}+I_{M}-2 a_{1} / b+l_{2}(j), \\
\sigma_{j} & =\pi^{2}\left(j-\frac{1}{2}\right)^{2}+I_{M}-2 a_{1} / b+l_{2}(j),
\end{aligned}
$$

where $I_{M}=2(a+d) / b+\int_{0}^{1} q d x$ is constant on $M$. Thus, since $I$ is finite, $a_{1}=a_{I}$.

To complete the proof, we only need show, for arbitrary $a_{1} \leq a_{I}$, sup $\left(a+a_{1}\right)=2 a_{1}$, where the supremum is taken over $M_{I} \cap\left\{d=a_{1}\right\}$. Let $\left(q_{N}, B_{N}\right)$ be the element of $M_{I}$ with

$$
\begin{gathered}
\mu_{j}\left(q_{N}, B_{N}\right) \equiv \sigma_{j}=\left\{\begin{array}{l}
\mu_{j}\left(q_{I}, B_{I}\right), \quad j \in I^{c} \cap\{j<N\}, \\
\mu_{j}\left(q_{I}, B_{I}\right)+\frac{2\left(a_{I}-a_{1}\right)}{b}, \quad j \in I^{c} \cap\{j \geq N\},
\end{array}\right. \\
y_{2}\left(1, \sigma_{j}, q_{N}\right)=y_{2}\left(1, \sigma_{j}, q_{I}\right), \quad j \in I^{c} \cap\{j<N\}
\end{gathered}
$$

and

$$
y_{2}\left(1, \sigma_{j}, q_{N}\right)=-b \xi_{*}\left(\sigma_{j}\right),
$$

where $\left|\xi_{*}\left(\sigma_{j}\right)\right| \leq 1$ for $j \in I^{c} \cap\{j \geq N\}$. To see that there is an element of $M$, and hence an element of $M_{l}$, with these coordinates, note that by $\S 3 \Phi(M) \supset N \cap$ $\left\{d \equiv d_{l}\right\}$, and by (5.12) there are points in $N \cap\left\{d=a_{1}\right\}$ with the given data. Since $a_{1} \leq a_{I}=d_{l}$, we see that $\left(q_{N}, B_{N}\right)$ exists and lies in $M_{I} \cap\left\{d=a_{1}\right\}$. Thus, setting $\mu_{j}=\mu_{j}\left(q_{l}, B_{l}\right), \mu_{j}^{N}=\mu_{j}\left(q_{N}, B_{N}\right)$ and $c=2\left(a_{l}-a_{1}\right) / b$, and applying (5.10) to 
$\left(q_{N}^{*}, B_{N}^{*}\right)$, we have for $N$ large

$$
\begin{aligned}
a_{N}+a_{1}= & 2 a_{I}-b^{2} \sum_{j=1}^{\infty}\left(\mu_{j}^{N}-\mu_{j}\right)\left(\prod_{i \neq j} \frac{\mu_{j}^{N}-\mu_{i}}{\mu_{j}^{N}-\mu^{N}}\right)\left[\frac{y_{2}+b \xi_{*}}{a_{I} y_{2}+b y_{2}^{\prime}}\right]\left(1, \mu_{j}^{N}, q_{I}\right) \\
= & 2 a_{I}-b^{2} \sum_{j \in I}\left(\mu_{j}^{N}-\mu_{j}\right)\left(\prod_{i \neq j} \frac{\mu_{j}^{N}-\mu_{i}}{\mu_{j}^{N}-\mu_{i}^{N}}\right)\left[\frac{y_{2}+b \xi_{*}}{a_{I} y_{2}+b y_{2}^{\prime}}\right]\left(1, \mu_{j}^{N}, q_{I}\right) \\
& -b^{2} c \sum_{j \geq N} \prod_{i \in I}\left(1+\frac{\mu_{i}^{N}-\mu_{i}}{\mu_{j}^{n}-\mu_{i}^{n}}\right) \prod_{\substack{i \geq N \\
j \neq i}}\left(1+\frac{c}{\mu_{j}-\mu_{i}}\right)\left[\frac{y_{2}+b \xi_{*}}{a_{I} y_{2}+b y_{2}^{\prime}}\right]\left(1, \mu_{j}^{N}, q_{I}\right) .
\end{aligned}
$$

Since the $\mu_{i}^{N}, i \in I$, lie in bounded intervals and $I$ is finite, (5.3) and (5.4) imply that the final sum in (5.13) tends to zero as $\cdot N \rightarrow \infty$. However, since $\left(q_{I}, B_{I}\right)$ has index $\mathbf{I}$,

$$
F_{j}(\mu, \xi)=\left(\mu-\mu_{j}\right)\left[\frac{y_{2}+b \xi}{a_{1} y_{2}+b y_{2}^{\prime}}\right]\left(1, \mu, q_{I}\right)
$$

is non-positive on $C_{j}$ for $j \in I$. Thus

$$
\liminf _{N \rightarrow \infty} a_{N}+a_{1} \geq 2 a_{1} \text {. }
$$

\section{The level sets of $B$ on $M$}

In this section we apply the results of the preceding sections to study isospectral sets when the boundary conditions are fixed. The subset of $M$ with boundary condition given by $B=\left(\begin{array}{ll}a & b \\ c & d\end{array}\right)$ will be denoted by $M_{B}$. Since $q \rightarrow q^{*}$ is an analytic homeomorphism of $M_{B}$ onto $M_{B}$, we only need consider the case $a \geq d$ for the results given here.

Proposition 4.7 implies that, when $a=d=a_{1}$ and $I=\varnothing$, then $M_{B}$ consists of a single point, and by theorem 5.1, if $a+d \geq 2 a_{\varnothing}$, then $M_{B}=\varnothing$ unless $a=d=a_{\varnothing}$. The first result of this section is that these are the only cases where $M_{B}$ is compact.

THEOREM 6.1. If $a+d<2 a_{\varnothing}$, then $M_{B}$ is not compact.

Proof. If $a+d<2 a_{\varnothing}$, then by theorem 5.1 there is a point $\left(q_{1}, B_{1}\right) \in M$ with $d_{1}=d$ and $a_{1}>a$. The strategy for proving this theorem is to show that using the flows from $V_{j}, j$ large, to move $a_{1}$ down to $a$, we can construct a non-compact set of points in $M_{B}$. By theorem 2.3 on the orbit of $\left(q_{1}, B_{1}\right)$ under the flow of $V_{j}$

$$
a(t)=a_{1}-\left(\mu_{j}(t)-\mu_{j}\right) b^{2}\left[\frac{y_{2}+b \xi_{*}}{a_{1} y_{2}+b y_{2}^{\prime}}\right]\left(1, \mu_{j}(t), q_{1}\right) .
$$

For $\left|\mu-\pi^{2}\left(j-\frac{1}{2}\right)^{2}\right|<C$ and $\left|\xi_{*}(\mu)\right|<1,(5.4)$ implies that

$$
\left[\frac{y_{2}+b \xi_{*}}{a_{1} y_{2}+b y_{2}^{\prime}}\right]\left(1, \mu, q_{1}\right)=O\left(\frac{1}{j^{2}}\right) \text {. }
$$

Thus, since $\mu_{j}\left(q_{1}, B_{1}\right)=\pi^{2}\left(j-\frac{1}{2}\right)^{2}+O(1)$, there is a $j_{0}$ such that for $j>j_{0}$, $\left|\mu-\mu_{j}\left(q_{1}, B_{1}\right)\right|<1$ and $\left|\xi_{*}(\mu)\right|<1$

$$
a_{1}-a<a_{1}-b^{2}\left(\mu-\mu_{j}\left(q_{1}, B_{1}\right)\right)\left[\frac{y_{2}+b \xi_{*}}{a_{1} y_{2}+b y_{2}^{\prime}}\right]\left(1, \mu, q_{1}\right) \text {. }
$$


On the other hand,

$$
\left[\frac{y_{2}+b \xi_{*}}{a_{1} y_{2}+b y_{2}^{\prime}}\right]\left(1, \lambda_{2 j}, q_{1}\right)=\frac{b(-1)^{j}+O(1 / j)}{b \cos \sqrt{\lambda_{2 j}}+O(1 / j)}=1+O\left(\frac{1}{j}\right)
$$

by (1.6). Thus there is a $j_{1}>j_{0}$ such that for $j>j_{1}$

$$
a_{1}-a>a_{1}-b^{2}\left(\lambda_{2 j}-\mu_{j}\left(q_{1}, B_{1}\right)\left[\frac{y_{2}+b \xi_{*}}{a_{1} y_{2}+b y_{2}^{\prime}}\right]\left(1, \lambda_{2 j}, q_{1}\right)\right. \text {. }
$$

Combining (6.1) and (6.3), we see that for each $j>j_{1}, M_{B}$ contains a point $\left(q_{j}, B\right)$ with

for $k \neq j$ and

$$
\mu_{k}\left(q_{j}, B\right)=\mu_{k}\left(q_{1}, B_{1}\right)
$$

$$
\left|\mu_{j}\left(q_{j}, B\right)-\mu_{j}\left(q_{1}, B_{1}\right)\right| \geq 1
$$

This implies that the image of $M_{B}$ under $\Phi$ is not compact and hence $M_{B}$ is not compact.

Since lemma 4.2(iv) and lemma 4.4 imply the gradient of $a$ on $M \cap\left\{d=d_{0}\right\}$ can vanish only at fixed points of the involution, and conversely one sees from lemma 4.2 (iv) and formula (5.1) with $(q, B)=\left(q_{I}, B_{I}\right)$ that $M_{B}$ does have a conic singularity at fixed points of the involution, we have the conclusion:

THEOREM 6.2. $M_{B}$ is an analytic submanifold of $L^{2}[0,1] \times(\operatorname{SL}(2, \mathbf{R}) \backslash b=0)$ unless it contains a fixed point of $*$; hence it is analytic unless $(a, d)=\left(a_{I}, d_{I}\right)$ for some $I \neq \varnothing$. If $(a, d)=\left(a_{I}, a_{I}\right)$ for some $I \neq \varnothing$, then $M_{B}$ has a conic singularity at $\left(q_{I}, B_{I}\right)$.

One property of $M_{B}$ which holds without exception is connectivity.

\section{THEOREM 6.3. $M_{B}$ is connected.}

Proof. Given any point $\left(q_{0}, B_{0}\right)$ in $M_{B_{0}}$ which is not fixed by the involution, there is a $j$ such that $\left(\partial a / \partial \mu_{j}\right)\left(\Phi\left(q_{0}, B_{0}\right)\right) \neq 0$ when we consider $a$ as a function on $N$. Thus, using the splitting $N=N_{I} \times N_{I}$ with $I=\{j\}$ and the implicit function theorem, we get $r(s)$ such that $\Phi^{-1}(r(s), s) \in M_{B_{0}}$ for $s$ in an open subset of $N_{I^{c}} \cap\left\{d=d_{0}\right\}$. Since points with

$$
\mu_{n}=\pi^{2}\left(n-\frac{1}{2}\right)^{2}+I_{M}-2 d_{0} / b
$$

and $\left|\kappa_{n}\right|<b$ for $n$ beyond some point are dense in $N_{I^{\circ}} \cap\left\{d=d_{0}\right\}$, it follows that we can connect $\left(q_{0}, B_{0}\right)$ to $\left(q_{1}, B_{0}\right)$ in $M_{B_{0}}$, where

$$
\mu_{n}\left(q_{1}, B_{0}\right)=\pi^{2}\left(n-\frac{1}{2}\right)^{2}+I_{M}-2 d_{0} / b
$$

and $\left|y_{2}\left(1, \mu_{n}, q_{1}\right)\right|<b$ for $n>n_{0}$.

Let $\left(q_{2}, B_{2}\right)$ be a point where $a$ assumes its maximum on the subset $S_{0}$ of $M$ satisfying (6.4). As in lemma 4.6 (see formula (4.3)), one sees that $a$ is strictly less than its maximum on $S_{0}$ away from $\left(q_{2}, B_{2}\right)$ and hence $\left(q_{2}, B_{2}\right)$ is unique. Moreover, $a$ has no other local maxima on $S_{0}$ and all its critical points on the torus $S_{0}$ must be non-degenerate and hence finite in number. Thus, if $\left(q_{1}, B_{0}\right) \neq\left(q_{2}, B_{0}\right)$, we may construct a continuous curve $\gamma(t)=(q(t), B(t))$ in $S_{0}$ with $\gamma(0)=\left(q_{1}, B_{0}\right)$ and $\gamma(1)=\left(q_{2}, B_{2}\right)$ such that $a$ is strictly increasing on $\gamma$. 
Next we will move the values of $a$ on $\gamma$ down to $a_{0}$ by using the flow from $V_{J}$, $J>n_{0}$ sufficiently large. Since $\{\gamma(t): 0 \leq t \leq 1\}$ is compact, it follows that the estimate (6.2) with $\left(a_{1}, q_{1}\right)$ replaced by $(a(t), q(t))$ and the corresponding estimate for $\mu=\lambda_{2 j-1}$ hold uniformly for $t \in[0,1]$. Moreover, as always, for each $t$ the function

$$
F_{j}(\mu, \xi ; t)=\left(\mu-\mu_{j}\right)\left[\frac{y_{2}+b \xi}{a(t) y_{2}+b y_{2}^{\prime}}\right](1, \mu, q(t))
$$

has a unique maximum and minimum on $C_{j}$ and no other critical points. Thus for $J$ sufficiently large we have two continuous functions $\mu_{ \pm}(t)$ with $\lambda_{2 J-1}<\mu_{-}(t) \leq$ $\mu_{+}(t)<\lambda_{2 J}$ such that

$$
a_{0}=a(t)-b^{2} F_{J}\left(\mu_{ \pm}(t), \xi\left(\mu_{ \pm}(t)\right) ; t\right)
$$

when $\mid \xi\left(\mu_{ \pm}(t) \mid<1\right.$. Thus, picking $\mu_{J}(t)=\mu_{*}(t)$ and $\kappa_{J}=-b \xi\left(\mu_{*}(t)\right)$, where $*(= \pm)$ is chosen so that $\mu_{*}(0)=\pi^{2}\left(J-\frac{1}{2}\right)^{2}+I_{M}-2 d_{0} / b$, and $\mu_{j}(t)=\mu_{j}(\gamma(t))$ and $\kappa_{j}(t)=$ $\kappa_{j}(\gamma(t))$ for $j \neq J$, we have a curve in $M_{B_{0}}$ connecting $\left(q_{1}, B_{0}\right)$ to either $\left(q_{2+}, B_{0}\right)$ or $\left(q_{2-}, B_{0}\right)$, where

$$
\begin{aligned}
& \mu_{j}\left(q_{2 \pm}, B_{0}\right)=\mu_{j}\left(q_{2}, B_{2}\right), \quad j \neq J, \\
& \mu_{J}\left(q_{2 \pm}, B_{0}\right)=\mu_{ \pm}(1) \text {, } \\
& \kappa_{j}\left(q_{2 \pm}, B_{0}\right)=\kappa_{j}\left(q_{2}, B_{2}\right) \quad \forall j .
\end{aligned}
$$

To see that $\left(q_{2+}, B_{0}\right)$ and $\left(q_{2-}, B_{0}\right)$ can be connected in $M_{B}$, we consider $a$ restricted to the two-dimensional torus $T_{0}$ obtained by fixing the coordinates on $M \cap\left\{d=d_{0}\right\}$ at their values in (6.5) for $j \neq J, J+1$. As before, $a$ has only one local maximum on $T_{0}$ and this is non-degenerate. Moreover, any other critical value for $a$ on $T_{0}$ must be a global minimum of $a$ on the orbit of the critical point under the flow of $V_{J}$ or $V_{J+1}$. Using the representation for $a$ on $T_{0}$ from theorem 2.4 with base point $\left(q_{2}, B_{2}\right)$ and moving on the flow of $V_{J}$ or $V_{J+1}$ to the point used in the proof of lemma 3.2, one sees that any critical value of $a$ on $T_{0}$ below the maximum must be below $a_{0}$ for $J$ sufficiently large. Thus the level set $a_{0}=a$ on $T_{0}$ which contains $\left(q_{2+}, B_{0}\right)$ and $\left(q_{2-}, B_{0}\right)$ is connected. Thus $\left(q_{1}, B_{0}\right)$ can be connected to both $\left(q_{2+}, B_{0}\right)$ and $\left(q_{2-}, B_{0}\right)$.

If $\left(q, B_{0}\right)$ and $\left(p, B_{0}\right)$ are points in $M_{B_{0}}$ which are not fixed by the involution, taking $n_{0}=\max \left\{n_{0}(q), n_{0}(p)\right\}$, we can assume $\left(q_{1}, B_{0}\right)$ and $\left(p_{1}, B_{0}\right)$ are both in $S_{0}$. Hence the preceding argument shows any two points of $M_{B_{0}}$ which are not fixed by the involution can be connected in $M_{B_{0}}$. Since fixed points of the involution are never isolated in $M_{B_{0}}$, we conclude $M_{B_{0}}$ is connected.

If we let $A=\left\{I: a_{0}+d_{0}>2 a_{I}\right\}$ and $B_{0}=\left(\begin{array}{cc}a_{0} & b \\ c_{0} & d_{0}\end{array}\right)$, then theorem 5.1 implies the $M_{B_{0}}$ is contained in

$$
S_{0}=\left(M-\bigcup_{I \in A} M_{l}\right) \cap\left\{d=d_{0}\right\} .
$$

We will conclude our study of $M_{B_{0}}$ with a theorem on the relation on the topological structure of $M_{B_{0}}$ to that of $S_{0}$. 
THEOREM 6.4. Assume that $a_{0}+d_{0} \neq 2 a_{l}$ for all $I$. Then the homotopy groups of $M_{B_{0}}$ and $S_{0}$ are isomorphic.

Proof. We will show that the natural homomorphism of the homotopy groups of $M_{B}$ into those of $S_{0}$ is injective and surjective. The proof of injectivity will have two main steps. We will show:

(i) If $\gamma: S^{m} \rightarrow M_{B_{0}}$, then given $j>j_{0}(\gamma), \gamma$ is homotopic in $M_{B_{0}}$ to $\tilde{\gamma}$, where for all $s \in S^{m}$ the roots of

$$
0=F_{j}(\mu, \xi ; \tilde{\gamma}(s))=\left(\mu-\mu_{j}(\tilde{\gamma}(s))\right)\left[\frac{y_{2}+\xi}{a_{0} y_{2}+b y_{2}^{\prime}}\right](1, \mu, q(\tilde{\gamma}(s)))
$$

on $C_{j}$ are contained in $|\xi|<1$ and $\mu=\mu_{j}(\tilde{\gamma}(s))$ is the greater root.

(ii) If $\Gamma: S^{m} \times[0,1] \rightarrow S_{0}$ is a homotopy of $\gamma$ in (i) to a constant map, then we can replace $\Gamma$ by a homotopy $\tilde{\Gamma}$ of $\gamma$ to a constant such that $\tilde{\Gamma}: S^{m} \times[0,1] \rightarrow S_{0} \cap$ $\left\{a \geq a_{0}\right\}$.

Given (i) and (ii), one can complete the proof of injectivity as follows. Choose $j_{1}>j_{0}(\gamma)$ sufficiently large that the range of $F_{j_{1}}(\mu, \xi ; \tilde{\Gamma}(s, t))$ on $C_{j} \cap\{|\xi|<1\}$ contains $\left(a_{0}-a(\tilde{\Gamma}(s, t))\right) b^{-2}$ for all $(t, s) \in[0,1] \times S^{m}$. Then extend $\tilde{\Gamma}$ to $S^{m} \times[-1,1]$ by letting $\tilde{\Gamma}$ on $S^{m} \times[-1,0]$ be the homotopy from (i) in $M_{B_{0}}$ of $\tilde{\gamma}$ with $j=j_{1}$ to $\gamma$. Finally, changing $\mu_{j_{1}}(\tilde{\Gamma}(s, t))$ to $\mu^{+}(s, t)$ as in the proof of theorem 6.3 gives a homotopy in $M_{B_{0}}$ of $\tilde{\gamma}$ to a constant and completes the proof of injectivity.

The proof that the mapping of the homotopy groups is surjective is similar and simpler. Given $f: S^{m} \rightarrow S_{0}$, we deform $f$ in $S_{0}$ to $\gamma: S^{m} \rightarrow S_{0} \cap\left\{a>a_{0}\right\}$ as in (ii) and then deform $\gamma$ in $S_{0} \cap\left\{a_{1}>a_{0}\right\}$ to $\tilde{\gamma}$ as in (i). Then moving $\mu_{j_{1}}(\tilde{\gamma}(s))$ continuously up to $\mu^{+}(s)$, we deform $\tilde{\gamma}$ to a curve in $M_{B_{0}}$. Note that this deformation automatically remains in $S_{0}$ since $a \geq a_{0}$. We believe that the proofs of the simpler versions of (i) and (ii) used here to prove surjectivity will be evident from the proofs of (i) and (ii).

Proof of (i). Since $M_{B_{0}}$ contains no critical points of $a+d$ on $M$ by hypothesis, and hence no critical points of $a$ on $M \cap\left\{d=d_{0}\right\}$, the gradient of $a$ as a function on $M \cap\left\{d=d_{0}\right\}$ does not vanish on $M_{B_{0}}$. Moreover, since $\gamma\left(S^{m}\right)$ is a compact subset of $M_{B_{0}}$, there is a finite set $I_{0}$ such that the gradient of $a$ as a function on $N_{l_{0}}$ does not vanish on $\gamma\left(S^{m}\right)$.

The idea of this proof is to use the $N_{I_{0}}$-gradient flow of $a$ to bring the deformation of $\gamma$ moving $\mu_{j}(\gamma(s))$ into $\mu_{j}(\tilde{\gamma}(s))$ back into $M_{B_{0}}$. However, getting the necessary uniform estimates in $j$ requires some additional work.

Using theorem 1.1 as in the derivation of (5.4), we have for $(\mu, \xi) \in C_{j} \cap\{|\xi|<1\}$

$$
\begin{aligned}
F_{j}(\mu, \xi ; q, B) & =\left(\mu-\mu_{j}(q, B)\right)\left[\frac{y_{2}+b \xi}{a y_{2}+b y_{2}^{\prime}}\right](1, \mu, q) \\
& =\left(\mu-\mu_{j}(q, B)\right)\left(\frac{b \cos \mu^{1 / 2}+O\left(\mu^{-1 / 2}\right)}{-b \mu^{1 / 2} \sin \mu^{1 / 2}+O(1)}\right),
\end{aligned}
$$

where both $O$ terms are uniform on bounded sets in $(\|q\|,\|B\|)$. Moreover, if denotes the derivative along a flow moving $q$ and $a$ but not $d, \Delta(\lambda, q, B)$ or $\mu_{j}(q, B)$, 
theorems 1.1, 1.2 and lemma 4.2 (iv) give

$$
\begin{aligned}
\left|\dot{F}_{j}(\mu, \xi ; q, B)\right|= & \mid\left(\mu-\mu_{j}(q, B)\right)\left[\frac{d\left(\partial y_{2} / \partial q, \dot{q}\right)+b\left(\partial y_{1} / \partial q, \dot{q}\right)}{y_{2}+b \xi^{-1}}\right. \\
& \left.-\frac{d y_{2}+b y_{1}}{\left(y_{2}+b \xi^{-1}\right)^{2}}\left(\frac{\partial y_{2}}{\partial q}, \dot{q}\right)\right](1, \mu, q) \mid \\
\leq & \frac{M(\|q\|,\|B\|)}{j}\left|\frac{\mu-\mu_{j}(q, B)}{y_{2}(1, \mu, q)+b \xi^{-1}(\mu)}\right|\|\dot{q}\|
\end{aligned}
$$

for $(\mu, \xi) \in C_{j} \cap\{|\xi|<1\}, j>j_{0}(\|q\|,\|B\|)$. In (6.6) we set

$$
\mu=\mu_{\delta}=\delta \pi\left(j-\frac{1}{2}\right)+\mu_{j}(q, B)
$$

with $|\delta| \leq 1$. Using theorem 1.3 , this gives for $\left|\xi_{\delta}\right|<1$

$$
F_{j}\left(\mu_{\delta}, \xi_{\delta} ; q, B\right)=\delta \tan \delta / 2+O(1 / j),
$$

uniformly on bounded sets in $(\|q\|,\|B\|, 1 / b)$ and $|\delta| \leq 1$. In (6.7) we consider $(\mu, \xi) \in D_{j}=C_{j} \cap\{|\xi|<1\} \cap\left\{\left|\mu-\mu_{j}\left(q_{j}, B\right)\right| \leq \pi\left(j-\frac{1}{2}\right)\right\}$ and conclude

$$
\left|\dot{F}_{j}(\mu, \xi ; q, B)\right| \leq \frac{C(\|q\|,\|B\|, 1 / b)}{j}\|\dot{q}\|
$$

for $j>j_{1}(\|q\|,\|B\|)$. The estimates (6.8) and (6.9) will suffice for this proof.

Since $F_{j}$ has just one maximum and one minimum on $C_{j}$ for all $(j, B, q)$, it is clear from $(6.8)$ that for any $\delta \in(0,1)$ and $j \geq j_{2}(\|q\|,\|B\|, 1 / b, 1 / \delta)$ the minimum of $F_{j}$ on $C_{j}$ must occur in

$$
I_{\delta}(j)=\left\{(\mu, \xi) \in C_{j}:\left|\mu-\mu_{j}(q, B)\right| \leq \delta \pi\left(j-\frac{1}{2}\right)\right\},
$$

and the range of $F_{j}$ on $I_{\delta}(j)$ is contained in $[-\delta, \delta]$. However, (6.8) also implies that for $j \geq j_{3}(\|q\|,\|B\|, 1 / b, 1 / \delta)$ the range of $F_{j}$ on $I_{j}(\delta)$ includes $[0, \delta / 2 \tan \delta / 2]$. Hence, taking $0<\varepsilon<\frac{1}{2} \tan \frac{1}{2}$, we can move along the flow of $V_{j}, j>$ $\max \left\{j_{i}(\|q\|,\|B\|, 1 / b, 2 / \varepsilon), i=2,3\right\}$, in the direction of increasing $\mu$ until $a=$ $a(q, B)-\varepsilon b^{2}$ at $\mu=\mu_{j}^{+}(q, B)$ and be sure that:

(i) $\mu_{j}^{+}(q, B)$ is greater than the minimum of $F_{j}(\mu, \xi ; \varepsilon, B)$ on $C_{j}$,

(ii) the values of $a$ in this process remain in $|a-a(q, B)|<4 \varepsilon b^{2}$ and $(\mu, \xi)$ remains in $D_{j}$. Since the flow is transverse to the level surface $a=a(q, B)-\varepsilon b^{2}$, this gives us smooth functions $\mu(t, q, B)$ and $t(q, B)$ such that $\mu_{j}(q, B)=\mu(0, q, B)$ and $\mu(t(q, B), q, B)=\mu_{j}^{+}(q, B)$. We set $\mu_{j}(\tau, q, B)=\mu(t(g, B) \tau, g, B)$ for

$$
j>\max \left\{j_{i}\left(\|q\|,\|B\|, \frac{1}{b}, \frac{2}{\varepsilon}\right), i=2,3\right\}=j_{4}\left(\|q\|,\|B\|, \frac{1}{b}, \frac{2}{\varepsilon}\right) .
$$

Since the $N_{I_{0}}$-gradient of a does not vanish on $\gamma\left(S^{m}\right)$, we may choose a neighbourhood $U$ in $N_{I_{0}}$ of the projection of $\gamma\left(S^{m}\right)$ onto $N_{I_{0}}$ such that the $N_{I_{0}}$-gradient of $a$ is bounded away from zero on $U$. Moreover, by (6.9) there is a $j_{S}(\gamma, U)$ such that the $N_{l_{0}}$-gradient of $a$ is bounded away from zero uniformly on

$$
\left.\left\{(r, u, v) \in N_{l_{0}} \times N_{\{j\}} \times N_{\left(I_{0} \cup\{j\}\right)^{c}}\right\}: r \in U, u \in D_{j}(\gamma(s)), v=v(\gamma(s)), s \in S^{m}\right\},
$$

uniformly in $j>j_{S}(\gamma, U)$. Hence we can choose an $\varepsilon>0$ such that for $j>j_{S}(\gamma, U)$ the $N_{I_{0}}$-gradient flow out of $E_{j}=\left\{(r, u, v) \in N_{t_{0}} \times N_{\{j\}} \times N_{\left(I_{0} \cup\{j\}\right)^{c}}: r=r(\gamma(s))\right.$, 
$\left.u \in D_{j}(\gamma(s)), v=v(\gamma(s))\right\}$ moves $a$ through $\left[a(e)-8 \varepsilon b^{2}, a(e)+8 \varepsilon b^{2}\right]$ for all $e \in E_{j}$ before $r$ leaves $U$. (We define the $N_{I_{0}}$-gradient flow of $a$ as the flow of the vector field $\sum_{j \in I_{0}} \dot{a}_{j} V_{j}$, where $\dot{a}_{j}$ denotes the derivative of $a$ on the flow of $V_{j}$ in (2.7).)

Now define

$$
(\alpha, \beta)=\left(\max _{G}\left\|q\left(\Phi^{-1}(r, u)\right)\right\|, \max \left\|B\left(\Phi^{-1}(r, u)\right)\right\|\right)
$$

where

$$
G=\left\{(r, u) \in N_{l_{0}} \times N_{I_{0}^{c}}: r \in N_{I_{0}}, u=u(\gamma(s)), s \in S^{m}\right\} .
$$

We let $j_{0}>j_{6}(\gamma)=\max \left\{j_{4}(\alpha, \beta, 1 / b, 2 / \varepsilon), j_{5}(\gamma, U)\right\}$. For such a $j_{0}$ we have a deformation $H(t, g)$ of $G$ given in our coordinates on $N$ by

$$
\begin{aligned}
& \mu_{j}= \begin{cases}\mu_{j}(\tau, g), & j=j_{0}, \\
\mu_{j}(g), & j \neq j_{0},\end{cases} \\
& \kappa_{j}= \begin{cases}-b \xi\left(\mu_{j}(\tau, g)\right),|\xi|<1, & j=j_{0}, \\
\kappa_{j}(g), & j \neq j_{0},\end{cases}
\end{aligned}
$$

such that for $(\tau, g) \in[0,1] \times G$ :

(i) $|a(H(\tau, g))-a(g)|<2 \varepsilon b^{2}$,

(ii) $a(H(1, g))=a(g)-\varepsilon b^{2}$,

(iii) $\mu_{j_{0}}(1, g)$ is greater than the value of $\mu$ at the minimum of $F_{j}(\mu, \xi ; g)$ on $C_{j}$. Moreover, letting $\phi_{r}$ be the $N_{t_{0}}$-gradient flow of $a$,

$$
a_{0}=a\left(H\left(\tau, \phi_{r}(\gamma(s))\right)\right)=a\left(\phi_{r}(H(\tau, \gamma(s)))\right)
$$

is uniquely solvable for $r(\tau, s)$ with $\phi_{r(\tau, s)} \in G$ for $(\tau, s) \in[0,1] \times S^{m}$. Now $F(s, \tau)=$ $H\left(\tau, \phi_{r(s, \tau)}(\gamma(s))\right)$ gives the homotopy of $\gamma$ required for step (i) with the $j_{0}(\gamma)$ in (i) given by $j_{6}(\gamma)$.

Proof of (ii). In proving (5.10) we used the assumption $(q, B) \in M_{I}$ only to insure that $\min \{a, d\} \leq a_{1}$. Hence (5.10) gives a representation of $a+d$ at a general point $(q, B)$ of $M$ in terms of the coordinates of $(q, B)$ when $d \leq a_{I}$. Since $F_{j}\left(\mu, \xi ; q_{I}, B_{I}\right)$ is positive on $C_{j}$ for $j \in I^{c}$ and negative on $C_{j}$ for $j \in I$, one sees as in (5.13) that for $d_{0} \leq a_{I}$

$$
\min _{N_{I}} \sup _{N_{I} \sim\left\{d=d_{0}\right\}} a \geq 2 a_{I}-d_{0} .
$$

Since $\Gamma$ maps $S^{m} \times[0,1]$ into a compact subset of $M \cap\left\{d=d_{0}\right\}$, there is a $k$ such that $\Gamma\left(S^{m} \times[0,1]\right) \cap M_{\{j\}}=\varnothing$ for $j>k$. Let $T$ denote the set of subsets $I$ of $\{1, \ldots, k\}$ such that $a_{0}+d_{0}<2 a_{I}$. Since $\Gamma\left(S^{m} \times[0,1]\right)$ is compact, one sees that the tails in (5.10) are uniformly small on $\Gamma\left(S^{m} \times[0,1]\right)$. Hence the proof of $(6.10)$ implies that there is a $l>k$ such that for all $I \in T$ and $(s, t) \in S^{m} \times[0,1]$

$$
\min _{r \in N_{I}} \max _{E_{l}(r, s, t)} a>2 a_{l}-d_{0}>a_{0},
$$

where

$$
E_{l}(r, s, t)=\left\{(u, v, w) \in N_{I} \times N_{l \cap \cap j \leq l\}} \times N_{\{j>l\}}: u=r, w=w(\Gamma(s, t))\right\} .
$$


In what follows we will work on the compact tori, parametrized by $S^{m} \times[0,1]$,

$$
N(s, t)=\left\{(u, v) \in N_{\{j \leq l\}} \times N_{\{j>l\}}: v=v(\Gamma(s, t))\right\} .
$$

Formula (6.11) will be the basis for the rest of this proof. We are going to imitate the proofs of Morse theory and use flows $\phi_{r}(s, t)$ on $N(s, t)$ closely related to the $N_{\{j \leq l\}}$-gradient flow of $a$ to deform $\Gamma$ into the set where $a>a_{0}$.

Given any finite subset $I$ of $Z_{+}$, we can repeat the construction of $r(s)$ in lemma 4.6 to get an analytic function $h(s)$ on $N_{I^{c}}$ such that $a(h(s), s)$ is the strict maximum of $a(h, s)$ for $h \in N_{I}$. Then we set

$$
S_{I}=\left\{\Phi^{-1}(h(s), s): s \in N_{I^{\mathrm{c}}}\right\}
$$

in analogy with $M_{l}$. Finally, for $I \subset\{1, \ldots, l\}$ and $(s, t) \in S^{m} \times[0,1]$ we set

$$
\begin{gathered}
M(s, t)=\Phi^{-1}(N(s, t)), \\
M_{I}(s, t)=M_{I} \cap \Phi^{-1}(N(s, t)), \\
S_{I}(s, t)=S_{I} \cap \Phi^{-1}(N(s, t)) .
\end{gathered}
$$

Now (6.11) can be rephrased as: for all $I \in T$ and $(s, t) \in S^{m} \times[0,1]$

$$
a\left(S_{I^{\mathrm{c}} \cap\{j \leq i\}}(s, t)\right)>a_{0} .
$$

Moreover, by hypothesis, for all $(s, t) \in S^{m} \times[0,1]$

$$
\Gamma(s, t) \notin M_{I}(s, t),
$$

when $I=\{j\}, k<j \leq l$, and when $I \subset\{1, \ldots, l\}$ and $a_{0}+d_{0}>2 a_{l}$.

As defined earlier, the $N_{\{j \leq l\}}$-gradient field of $a$ is given by

$$
V(q, B)=\sum_{j=1}^{l} \dot{a}_{j}(q, B) V_{j}(q, B),
$$

where $\dot{a}_{j}$ denotes $\dot{\boldsymbol{a}}$ on the flow of $V_{j}$. The flow of this vector field leaves $M(s, t)$ invariant and $\dot{a} \geq 0$ on the flow, vanishing only at zeros of $V$. If $(q, B)$ is a zero of $V$ on $M(s, t)$, then for some set $J \subset\{1, \ldots, l\}$

$$
(q, B)=M_{J}(s, t) \cap S_{J}(s, t),
$$

where $J^{\prime}=\{1, \ldots, l\} \cap J^{c}$. By (6.13), $\Gamma(s, t)$ can be a zero of $V$ only if $J$ in (6.14) belongs to $T$ - note that $M_{I}(s, t) \cap M_{I}(s, t)=M_{I \cup I}(s, t)-$ and hence $a(\Gamma(s, t))>a_{0}$ by (6.12). However, this does not imply that the flow of $V$ will eventually make $a>a_{0}$. In what follows on $M(s, t)$ we change $V$ to $V(s, t)$, depending continuously on $(s, t)$, with the following properties:

(a) $V(s, t)$ has the same zeros as $V$ on $N(s, t)$ and $a$ is non-decreasing on $V(s, t)$.

(b) $V(s, t)$ is tangent to $M_{I}(s, t)$ for all $I \subset\{1, \ldots, l\}$.

(c) If $(q, B)=M_{J}(s, t) \cap S_{J}(s, t)$ is a zero of $V$, then $M_{J}(s, t)$ contains the stable manifold of $(q, B)$ under the flow of $V(s, t)$.

(d) $V(s, 1)$ is independent of $s$.

Since $S^{m} \times[0,1]$ is compact and all dependence on $(s, t)$ is continuous, it follows from (6.12), (6.13), (6.14) and (a)-(d) that, letting $\phi_{r}\left(q, B_{j} s, t\right)$ denote the flow of $V(s, t)$, there is an $R$ such that for all $(s, t) \in S^{m} \times[0,1]$

$$
a\left(\phi_{R}(\Gamma(s, t) ; s, t)\right)>a_{0} .
$$


Given (6.15), we can build $\tilde{\Gamma}$ by defining

$$
\begin{aligned}
\Gamma_{1}(s, t) & = \begin{cases}\Gamma(s, 0), & 0 \leq t \leq \frac{1}{2}, \\
\Gamma(s, 2 t-1), & \frac{1}{2} \leq t \leq 1,\end{cases} \\
r(t) & = \begin{cases}2 t R, & 0 \leq t \leq \frac{1}{2}, \\
R, & \frac{1}{2} \leq t \leq 1,\end{cases} \\
v(t) & = \begin{cases}0, & 0 \leq t \leq \frac{1}{2}, \\
2 t-1, & \frac{1}{2} \leq t \leq 1,\end{cases} \\
\tilde{\Gamma}(s, t) & =\phi_{r(t)}\left(\Gamma_{1}(s, t) ; s, v(t)\right)
\end{aligned}
$$

Hence this proof will be complete once we construct $V(s, t)$.

The construction of $V(s, t)$ is based on facts already used in proving lemma 4.6: if $(q, B) \in M_{I} \cap S_{I^{\prime}}, I \cap I^{\prime}=\varnothing$, then $\left(\partial^{2} a / \partial \tilde{\mu}_{i} \partial \tilde{\mu}_{j}(\Phi(q, B))\right), i, j \in I \cup I^{\prime}$, is a diagonal matrix with $\partial^{2} a / \partial \tilde{\mu}_{i}^{2}>0$ for $i \in I$ and $\partial^{2} a / \partial \tilde{\mu}_{i}^{2}<0$ for $i \in I^{\prime}-$ we allow $I$ or $I^{\prime}$ to be empty. As in lemma 4.6, an implicit function argument implies that locally one has an analytic function $\tilde{r}: N_{\left(I \cup I^{\prime}\right)^{c}} \rightarrow N_{I \cup I^{\prime}}$ so that $\Phi\left(M_{I} \cap S_{I}\right)$ is given by $(\tilde{r}(u), u)$. If we choose $r(u)$ so that

$$
\max _{v \in N_{,}} a(h(u, v), v, u)
$$

is assumed at $(h(u, v), v)=r(u)$, where $\Phi\left(M_{I}\right)$ is given by $(h(w), w)$, it follows that $\Phi^{-1}(r(u), u) \in M_{I} \cap S_{I^{\prime}}$, and the argument used to prove proposition 4.7 shows $r(u)$ is unique. Hence $r(u)=\tilde{r}(u)$ and is analytic. Now setting $R_{I, I^{\prime}}=M_{I} \cap S_{I^{\prime}}$, we have

$$
\left.R_{I, I^{\prime}}=\Phi^{-1}\left(\{r(u), u): u \in N_{\left(I \cup I^{\prime}\right)^{c}}\right\}\right),
$$

generalizing the constructions of $M_{J}$ and $S_{j}$. Hence for $I, I^{\prime} \subset\{1, \ldots, l\}$, setting

$$
R_{I, r}(s, t)=R_{I, I} \cap M(s, t)=M_{I}(s, t) \cap S_{I}(s, t),
$$

we see that $\Phi\left(R_{I, I}(s, t)\right)$ is an analytic subset of $N(s, t)$, given globally by $r: N_{(I \cup I \mid)^{c} \cap\{j \leq l\}} \rightarrow N_{I \cup I^{\prime}}$, where $r$ also depends continuously on $(s, t)$. In the case $I \cup I^{\prime}=\{1, \ldots, l\}$, if $(q, B)=R_{I, I}(s, t)$, the fact that $\left(\partial^{2} a / \partial \tilde{\mu}_{i} \partial \tilde{\mu}_{j}(\Phi(q, B))\right), 1 \leq i$, $j \leq l$, is diagonal implies that the tangent spaces satisfy

$$
\begin{array}{ll}
T_{(q, B)}\left(M_{\{j\}}(s, t)\right)=\operatorname{span}\left\{V_{i}(q, B): i \neq j, i \leq l\right\} & \text { for } j \in I, \\
T_{(q, B)}\left(S_{\{j\}}(s, t)\right)=\operatorname{span}\left\{V_{i}(q, B): i \neq j, i \leq l\right\} & \text { for } j \in I^{\prime} .
\end{array}
$$

Moreover, $\partial^{2} a / \partial \tilde{\mu}_{i}^{2}>0, i \in I$, and $\partial^{2} a / \partial \tilde{\mu}_{i}^{2}<0, i \in I^{\prime}$, imply that the $N_{\{j \leq l\}}$-gradient flow of $a$ on $M(s, t)$ has a hyperbolic fixed point at $(q, B)$, with stable manifold tangent to $M_{I}(s, t)$ at $(q, B)$ and unstable manifold tangent to $S_{I}(s, t)$ at $(q, B)$.

We let $V$ denote the $N_{\{j \leq l\}}$-gradient field of $a$ as before, and begin by constructing $V(s, t)$ near the zeros of $V$. As noted earlier, the zeros of $V$ on $M(s, t)$ are the union of the distinct (since we ignore degenerate gaps) points $R_{J, J^{\prime}}(s, t)$, where $J, J^{\prime}$ range over disjoint sets satisfying $J \cup J^{\prime}=\{1, \ldots, l\}$. In view of (6.16), we can introduce coordinates $n_{1}, \ldots, n_{l}$ near $R_{J, J^{\prime}}(s, t)$, continuously in $(s, t)$, so that $n_{j}=0$ on $M_{\{j\}}(s, t), j \in J$, and $n_{j}=0$ on $S_{\{j\}}(s, t), j \in J^{\prime}$, and the Jacobian $\left(\partial n_{j} / \partial \tilde{\mu}_{k}\right)$ will be diagonal at $R_{J, J}(s, t)$. Let $V_{n_{j}}$ denote the vector field tangent to the curves $\gamma\left(t, n_{0}\right)$ 
given by $n_{k}=n_{k}^{0}, k \neq j, n_{j}=n_{j}^{0}+t$. Near $R_{J, J}(s, t)$ we set

$$
X_{J}(s, t)=\sum_{j=1}^{t} c_{j}(s, t) n_{j} V_{n_{j}}
$$

where the coefficients $c_{j}(s, t)$ are chosen so that $X_{j}(s, t)-V$ vanishes to second order at $R_{J, J}(s, t)$. The vector field $X_{J}(s, t)$ is tangent to all the manifolds $M_{l}(s, t)$ passing through $R_{J, J}(s, t)$, and $\dot{a}$ will be strictly positive on the flow of $X_{J}(s, t)$ on a punctured neighbourhood of $R_{J, J}(s, t)$. Hence we can choose a cut-off $\phi$ on $N(s, t)$ depending continuously on $(s, t)$ such that $\phi=1$ near $R_{J, J}(s, t), \phi(s, 1)$ is independent of $s$, and $\dot{a}$ will be non-negative on the flow of

$$
W_{J}(s, t)=\phi(s, t) X_{J}(s, t)
$$

on all of $N(s, t)$.

The vector fields $W_{J}(s, t)$ have the properties we require near the points $R_{J, J^{c} \cap\{j \leq l\}}(s, t)$. Now we need a vector field tangent to all the $M_{J}(s, t), J \subset\{1, \ldots, l\}$, such that $\dot{a}>0$ on this vector field outside a small neighbourhood of the $R_{J, J^{\prime} \cap\{j \leq l\}}(s, t)$. To build this, we consider the set $\mathscr{C}$ of all subsets of $\{1, \ldots, l\}$ and let $\mathscr{C}_{j}$ denote the subset of $\mathscr{C}$ with $\#\{I\}=j$. Given $I \in \mathscr{C}_{n}$, we set

$$
U_{I}(s, t)=\sum_{j \in I^{\mathbf{x}} \cap\{j \leq I\}} \dot{a}_{j} V_{j}+\sum_{j \in I} b_{j}(s, t) V_{j}
$$

on $M_{l}(s, t)$, where the $b_{j}$ are uniquely determined by the requirement that $V_{I}(s, t)$ is tangent to $M_{I}(s, t)$. We are going to take $V(s, t)=U_{I}(s, t)$ on $M_{I}(s, t)$ outside a small neighbourhood of

$$
K_{n}(s, t)=\left(\bigcup_{j>n} \bigcup_{I \in \mathscr{C}_{j}} M_{I}(s, t)\right) \cup\left(\bigcup_{J \subset\{1, \ldots, t\}} R_{J, J^{\prime} \cap\{j \leq l\}}(s, t)\right) .
$$

Note that $V(s, t)$ is well defined on $M_{l}(s, t)$ since $M_{I}(s, t) \cap M_{J}(s, t)=M_{I \cup J}(s, t)$ and $\#\{I \cup J\}>n$ unless $I \supset J$. Moreover, on $M_{I}(s, t), \dot{a}=\sum_{i \in I^{c} \cap(j \leq l)}\left(\dot{a}_{i}\right)^{2}$ and this is strictly positive on $M_{I}(s, t)$ off $\bigcup_{J \subset\{1, \ldots, l\}} R_{J, J^{c} \cap\{j \leq l\}}(s, t)$. We extend $U_{I}(s, t)$ continuously in $(s, t)$ to a neighbourhood of $M_{l}(s, t)$ in $N(s, t)$ so that it is tangent to $M_{\{j\}}(s, t), j \in I$, and then set

$$
Y_{I}(s, t)=\phi U_{I}(s, t),
$$

where $\phi=1$ on $M_{I}(s, t)$ off a sufficiently small neighbourhood of $K_{n}(s, t), \phi=0$ on a neighbourhood of $K_{n-1}(s, t) \backslash M_{I}(s, t)$, and $\phi$ also vanishes outside a sufficiently small neighbourhood of $M_{I}(s, t)$. Choosing the 'sufficiently small' neighbourhoods here first for $n=l-1$, then for $n=l-2$ and so on, we can, for each $n>0$, make

$$
Z_{n}(s, t)=\sum_{j=n}^{l-1} \sum_{I \in \mathscr{C}_{j}} Y_{I}(s, t)+\sum_{J \in\{1, \ldots, l\}} W_{J}(s, t)
$$

tangent to $M_{l}(s, t)$ for all $I$ and make $\dot{a} \geq 0$ everywhere and $\dot{a}>0$ on

$$
\left(\bigcup_{j=n}^{l-1} \bigcup_{I \in \mathscr{C}_{j}} M_{I}(s, t)\right) \backslash\left(\bigcup_{J \in\{1, \ldots, l\}} R_{J, J^{c} \cap\{j \leq l\}}(s, t)\right)
$$

on the flow of $Z_{n}(s, t)$. Moreover, we can choose the cut-offs $\phi$ to depend continuously on $(s, t)$ and be independent of $s$ when $t=1$. 
Now we conclude by setting

$$
V(s, t)=Z_{1}(s, t)+\phi V
$$

where $\phi$ is a cut-off vanishing on a neighbourhood of $K_{0}(s, t)$ with $\phi=1$ off a sufficiently small neighbourhood of $K_{0}(s, t)$, and we have a vector field with the desired properties.

Remark 6.5. In the proof of the injectivity of the homomorphism of the homotopy groups of $M_{B_{0}}$ into those of $S_{0}$, it is not necessary that the deformation of $\Gamma$ into $\tilde{\Gamma}$ in step (ii) be done in $S_{0}$. However, it is essential that the corresponding deformation in the proof of the surjectivity of the homomorphism remain in $S_{0}$. Hence it is important to note that the deformation in the preceding proof can be done in $S_{0}$. To see this, recall that, given $(q, B)$ in $M$, there is a $j_{0}$ such that for $j>j_{0}$ the coordinate $\kappa_{j}$ satisfies $\left|\kappa_{j}\right|>b$ at the minimum of $a$ on the orbit of $(q, B)$ in the flow of $V_{j}$. Since the minimum of $a$ on this orbit never occurs when $\mu_{j}=\lambda_{2 j}$ or $\mu_{j}=\lambda_{2 j-1}$ (cf. lemma 4.3), it follows that, for $j>j_{0},\left|\kappa_{j}\right|>b$ on $M_{\{j\}}$. Since $\Gamma\left(S^{m} \times[0,1]\right)$ (or $f\left(S^{m}\right)$ when one is proving surjectivity) is compact, it follows that there is a $j_{1} \geq j_{0}$ such that $\left|\kappa_{j}\left(\Gamma\left(S^{m} \times[0,1]\right)\right)\right|<b$ for $j>j_{1}$. Choosing $k$ in the preceding proof larger than $j_{1}$, one sees that

$$
N(s, t) \cap M_{J}=\phi
$$

for all $(s, t) \in S^{m} \times[0,1]$ unless $J \subset\{1, \ldots, l\}$. Since the flows used in the proof preserve $M_{J}$ for $J \subset\{1, \ldots, l\}$, it follows that the deformation remains in $S_{0}$.

Appendix A. Boundary conditions of the form

$$
\left(\begin{array}{cc}
a & 0 \\
c & 1 / a
\end{array}\right)\left(\begin{array}{l}
y(1) \\
y^{\prime}(1)
\end{array}\right)=\left(\begin{array}{l}
y(0) \\
y^{\prime}(0)
\end{array}\right) .
$$

1. Asymptotics. We will first discuss the asymptotic behaviour of the eigenvalues $\lambda_{m}, n=0,1, \ldots$, for these problems. For $a \neq 1$ the techniques in $\S 1$ can be used to obtain

$$
\begin{array}{ll}
\lambda_{2 n}=n^{2} \pi^{2}+2 n \pi k+k^{2}+\frac{2 c}{a+a^{-1}}+\int_{0}^{1} q(t) d t+l^{2}(n), & n \geq 0, \\
\lambda_{2 n-1}=n^{2} \pi^{2}-2 n \pi k+k^{2}+\frac{2 c}{a+a^{-1}}+\int_{0}^{1} q(t) d t+l^{2}(n), & n \geq 1,
\end{array}
$$

where $k=\cos ^{-1}(2 /(a+1 / a))$. This means that the $n$th gap length is

$$
\lambda_{2 n}-\lambda_{2 n-1}=4 k \pi n+l^{2}(n), \quad n \geq 1,
$$

and the $n$th band length

$$
\lambda_{2 n-1}-\lambda_{2 n-2}=2(\pi-2 k) \pi n-\pi^{2}+2 \pi k+l^{2}(n), \quad n \geq 1 .
$$

We note that if $k \in(0, \pi / 4)$, the bands grow faster than the gaps, and if $k \in$ $(\pi / 4, \pi / 2)$, the gaps grow faster than the bands.

The case $a=1$ is different and has more resemblance to the case of periodic boundary conditions. To find the asymptotics of the eigenvalues, one can here use the refined estimates of $y_{1}, y_{2}$, and $y_{2}^{\prime}$ discussed immediately following theorem 1.3 
in [6]. We find that for $\lambda \in \mathbf{R}^{+}$

$$
\begin{aligned}
y_{1}(1, \lambda, q)= & \cos \sqrt{\lambda}+\frac{\sin \sqrt{\lambda}}{2 \sqrt{\lambda}} \int_{0}^{1} q(t) d t+\frac{\sin \sqrt{\lambda}}{2 \sqrt{\lambda}} \int_{0}^{1} \cos (\sqrt{\lambda} 2 t) q(t) d t \\
& -\frac{\cos \sqrt{\lambda}}{2 \sqrt{\lambda}} \int_{0}^{1} \sin (\sqrt{\lambda} 2 t) q(t) d t-\frac{\cos \sqrt{\lambda}}{8 \lambda}\left(\int_{0}^{1} q(t) d t\right)^{2}+R_{1}(\lambda) \\
& y_{2}(1, \lambda, q)=\frac{\sin \sqrt{\lambda}}{\sqrt{\lambda}}-\frac{\cos \sqrt{\lambda}}{2 \lambda} \int_{0}^{1} q(t) d t+R_{2}(\lambda) \\
y_{2}^{\prime}(1, \lambda, q)= & \cos \sqrt{\lambda}+\frac{\sin \sqrt{\lambda}}{2 \sqrt{\lambda}} \int_{0}^{1} q(t) d t-\frac{\sin \sqrt{\lambda}}{2 \sqrt{\lambda}} \int_{0}^{1} \cos (\sqrt{\lambda} 2 t) q(t) d t \\
& +\frac{\cos \sqrt{\lambda}}{2 \sqrt{\lambda}} \int_{0}^{1} \sin (\sqrt{\lambda} 2 t) q(t) d t-\frac{\cos \sqrt{\lambda}}{8 \lambda}\left(\int_{0}^{1} q(t) d t\right)^{2}+R_{3}(\lambda)
\end{aligned}
$$

where $R_{1}, R_{2}$, and $R_{3}$ are $O(1 / \lambda)$. Using Rouché estimates on $y_{1}, y_{2}$ and $y_{2}^{\prime}$, we find that for $\lambda=\lambda_{2 n-1}$ or $\lambda_{2 n}, \sqrt{\lambda}=n \pi+r_{n}$ with $r_{n}=O(1 / n)$. For a sequence $\left\{\lambda_{n}\right\}_{1}^{\infty}$ with such asymptotics we have

$$
\left\{R_{i}\left(\lambda_{n}\right)\right\}_{1}^{\infty} \in l_{2}^{2}\left(\mathbf{Z}^{+}\right), \quad i=1,2,3,
$$

where $l_{k}^{2}\left(\mathbf{Z}^{+}\right)$is the set of all sequences $\left\{a_{n}\right\}_{1}^{\infty}$ with $\left\{n^{k} a_{n}\right\}_{1}^{\infty} \in l^{2}\left(\mathbf{Z}^{+}\right)$. This is seen by observing that in this case the remainder term consists of sums of Fourier coefficients of $q$ multiplied by $O\left(1 / \lambda_{n}\right)$ plus terms which are $O\left(1 / \lambda_{n}^{3 / 2}\right)$.

We recall that the eigenvalues $\lambda_{2 n-1}$ and $\lambda_{2 n}$ are roots of the equation

$$
F(\lambda)=a y_{1}(1, \lambda)+c y_{2}(1, \lambda)+(1 / a) y_{2}^{\prime}(1, \lambda)=2(-1)^{n},
$$

which in the case $a=1$ reduces to

$$
y_{1}(1, \lambda)+c y_{2}(1, \lambda)+y_{2}^{\prime}(1, \lambda)=2(-1)^{n} .
$$

Now using the asymptotics for $y_{1}, y_{2}$ and $y_{2}^{\prime}$ stated above, we see that the terms involving the Fourier coefficients of $q$ in (A.1(i)) and (A.1(iii)) cancel in the equation for the eigenvalues precisely when $a=1$. Setting $\sqrt{\lambda}=n \pi+r_{n}$, where $\lambda=\lambda_{2 n-1}$ or $\lambda_{2 n}$, we derive the equation

$$
\begin{aligned}
2 \cos r_{n}= & 2-2 \frac{\sin r_{n}}{2\left(n \pi+r_{n}\right)} \int_{0}^{1} q(t) d t+2 \frac{\cos r_{n}}{8\left(n \pi+r_{n}\right)^{2}}\left(\int_{0}^{1} q(t) d t\right)^{2} \\
& -c \frac{\sin r_{n}}{n \pi+r_{n}}+c \frac{\cos r_{n}}{2\left(n \pi+r_{n}\right)^{2}} \int_{0}^{1} q(t) d t+l_{2}^{2}(n) .
\end{aligned}
$$

We now divide this equation by $2 \cos r_{n}$ and solve the second-order equation in $r_{n}$ that we obtain by deleting terms in $l_{2}^{2}\left(Z^{+}\right)$. For $c \geq 0$ this gives us the asymptotics

$$
\begin{array}{ll}
\lambda_{2 n}=n^{2} \pi^{2}+2 c+\int_{0}^{1} q d t+l^{2}(n), & n \geq 0, \\
\lambda_{2 n-1}=n^{2} \pi^{2}+\int_{0}^{1} q d t+l^{2}(n), & n \geq 1,
\end{array}
$$


and for $c<0$

$$
\begin{gathered}
\lambda_{2 n}=n^{2} \pi^{2}+\int_{0}^{1} q d t+l^{2}(n), \quad n \geq 0, \\
\lambda_{2 n-1}=n^{2} \pi^{2}+2 c+\int_{0}^{1} q d t+l^{2}(n), \quad n \geq 1 .
\end{gathered}
$$

The $n$th gap length is therefore

$$
\lambda_{2 n}-\lambda_{2 n-1}=2|c|+l^{2}(n), \quad n \geq 1,
$$

and the $n$th band length

$$
\lambda_{2 n-1}-\lambda_{2 n-2}=2 n \pi-\pi^{2}-2|c|+l^{2}(n), \quad n \geq 1 .
$$

2. Isospectral flows. The theorems and arguments of this section are completely analogous to those of $\S 2$. We will therefore just state our results.

We fix $p \in L_{\mathbf{R}}^{2}[0,1]$ and define the isospectral set

$$
L(p)=\left\{q \in L_{\mathbf{R}}^{2}[0,1]: \lambda_{n}(q)=\lambda_{n}(p), n \geq 1\right\} .
$$

To study the geometric nature of $L(p)$ we first construct vector fields on $L(p)$ :

THEOREM. Let $p \in L_{\mathbf{R}}^{2}[0,1]$. Then for each $\lambda \in \mathbf{R}$ the vector field

$$
Z_{\lambda}(q)=2\left(\frac{\partial \Delta(\lambda, q)}{\partial q(x)}\right)^{\prime}
$$

is tangent to $L(p)$. That is, a solution of the differential equation

$$
\dot{q}(x, t)=\left[Z_{\lambda}(q)\right](x, t)
$$

with initial data in $L(p)$ stays in $L(p)$ for all time.

As in $\S 1$, we can map the isospectral set $L(p)$ into a space $S$ consisting of sequences of the form

$$
\left(\mu_{1}, \kappa_{1}, \mu_{2}, \kappa_{2}, \ldots\right)
$$

where

$$
\mu_{n}=n^{2} \pi^{2}+\int_{0}^{1} p d t+l^{2}(n), \quad \kappa_{n}=l_{1}^{2}(n) .
$$

From theorems 3.5 and 3.6 of [6] we know that the map

$$
\phi: L_{\mathbf{R}}^{2}[0,1] \rightarrow S
$$

given by

$$
\begin{aligned}
\phi= & \left(\mu_{1}(q), \log (-1) y_{2}^{\prime}\left(1, \mu_{1}(q), q\right), \mu_{2}(q),\right. \\
& \left.\log (-1)^{2} y_{2}^{\prime}\left(1, \mu_{2}(q), q\right), \ldots, \mu_{n}(q), \log (-1)^{n} y_{2}^{\prime}\left(1, \mu_{n}(q), q\right), \ldots\right)
\end{aligned}
$$

is an analytic homeomorphism of $\left\{q \in L_{\mathbf{R}}^{2}[0,1): \int_{0}^{1} q d t=\int_{0}^{1} p d t\right\}$ and the sequence space $S$ with the $l^{2}$-coordinatization given in [6]. Using the definition of $\Delta$,

$$
\Delta\left(\mu_{n}\right)=a y_{1}\left(1, \mu_{n}\right)+(1 / a) y_{2}^{\prime}\left(1, \mu_{n}\right),
$$

and the Wronskian identity

$$
y_{1}\left(1, \mu_{n}\right)=1 / y_{2}^{\prime}\left(1, \mu_{n}\right)
$$


we derive the equation

$$
\left(y_{2}^{\prime}\left(1, \mu_{n}\right)\right)^{2}-a \Delta\left(\mu_{n}\right) y_{2}^{\prime}\left(1, \mu_{n}\right)+a^{2}=0,
$$

which we can write in terms of the $\kappa_{n}$ as

$$
\left(e^{\kappa_{n}}\right)^{2}-a \Delta\left(\mu_{n}\right)(-1)^{n} e^{\kappa_{n}}+a^{2}=0 .
$$

Thus $\phi(L(p))$ is contained in a product of real analytic curves which are topologically circles.

Let

$$
p_{n}(q)=\left(\mu_{n}(q), \kappa_{n}(q)\right), \quad n \geq 1
$$

and let $\sigma_{n}(q) \in\{+,-\}$ denote the sign of the radical in the equation

$$
a y_{1}\left(1, \mu_{n}(4), q\right)=\Delta\left(\mu_{n}\right) / 2 \pm \frac{1}{2}\left(\Delta^{2}\left(\mu_{n}\right)-4\right)^{1 / 2} \text {. }
$$

Now let $Z_{n}$ denote the vector fields $Z_{\lambda}$ with $\lambda=\mu_{n}(q(\cdot, t))$. For these vector fields the integral curves have a very simple description in terms of the $p_{n}$ as is seen by the following result.

THEOREM. Under the flow of $Z_{n}$ the points $p_{m}(q(\cdot, t)), m \neq n$, remain fixed on their circles, while the point $p_{n}(q(\cdot, t))$ moves clockwise around its circle. It moves in such a way that

$$
d \mu_{n}(q(\cdot, t)) / d t=\sigma_{n}(q(\cdot, t)) \cdot\left(\Delta^{2}\left(\mu_{n}\right)-4\right)^{1 / 2} .
$$

We can in fact integrate the vector fields $Z_{n}$ explicitly. Thus we will be able to see directly how a potential changes as we move along an integral curve of $Z_{n}$. The explicit integration of $Z_{n}$ is given by the following theorem, analogous to theorem 2.3 .

THEOREM. Let $q_{0} \in L(p)$ and let $\mu_{n}(t)$ denote the unique solution of

$$
\frac{d}{d t} \mu_{n}(t)= \pm\left(\Delta^{2}\left(\mu_{n}(t)\right)-4\right)^{1 / 2}
$$

for which the point $p_{n}(t)=\left(\mu_{n}(t), \kappa_{n}(t)\right)$ starts at $p_{n}\left(q_{0}\right)$ and moves clockwise around its circle without pausing. Here the sign in front of the radical is chosen to be $\sigma_{n}\left(q_{0}\right)$ for $t=0$ and then to change when the radical vanishes. Then the integral curve of $Z_{n}$ passing through $q_{0}$ is given by

$$
q(x, t)=q_{0}(x)-2 \frac{d^{2}}{d x^{2}} \log \left[f_{\sigma_{n}(i)}\left(\mu_{n}(t), q_{0}\right), y_{2}\left(\mu_{n}, q_{0}\right)\right],
$$

where $f_{ \pm}(x, \lambda, q)$ are the solutions of $-u^{\prime \prime}+q u=\lambda u$ associated with eigenvectors of $B F(\lambda)$ as in $\S 2$.

3. Geometry of $L(p)$. We are now in a position to describe $L(p)$ as a submanifold of $L_{\mathbf{R}}^{2}[0,1]$. As in $\S 3$, we will proceed by determining the image of $L(p)$ in the sequence space $S$ under the coordinate map $\phi$.

As noted earlier, the coordinates $\left(\mu_{1}, \kappa_{1}, \mu_{2}, \kappa_{2}, \ldots\right)$ of a point in $L(p)$ satisfy the equations

$$
e^{2 \kappa_{n}}+(-1)^{n+1} a \Delta\left(\mu_{n}\right) e^{\kappa_{n}}+a^{2}=0, \quad n \geq 1 .
$$

Let $N$ be the subset of $S$ defined by these equations. We claim that $N$ is a real 
analytic submanifold of $S$. From the properties of $\Delta$ it follows that for each $n$ equation (A.3) defines a real analytic curve which topologically is a circle in the $\left(\mu_{n}, \kappa_{n}\right)$-plane unless $\lambda_{2 n-1}=\lambda_{2 n}$, in which case the circle degenerates to a point. From the asymptotics of the $\lambda$ it follows that this degeneracy can only occur a finite number of times. We may give $N$ locally as an explicit submanifold of $S$ by solving (A.3) for $\mu_{n}$ or $\kappa_{n}$ as appropriate for each $n$ for which the corresponding circle is non-degenerate. In the case $a \neq 1$ the asymptotics of

$$
\Delta\left(\mu_{n}\right)=a y_{1}\left(1, \mu_{n}\right)+\frac{1}{a} y_{2}^{\prime}\left(1, \mu_{n}\right)=(-1)^{n}\left(a+\frac{1}{a}\right)+O\left(\frac{1}{n}\right)
$$

imply that there is an $n_{0}$ such that for $n>n_{0}$

$$
\kappa_{n}=\log \left[\frac{(-1)^{n} \dot{a} \Delta\left(\mu_{n}\right)}{2}-\left(\frac{a^{2} \Delta^{2}\left(\mu_{n}\right)}{4}-a^{2}\right)^{1 / 2}\right]
$$

on the intersection of $N$ with a bounded subset of $S$. Thus $N$ is qualitatively the same as $N \cap\left\{d=d_{0}\right\}$ in $\S 3$ and, since the proof that $N$ is real analytic exactly follows the proof given there, we will not repeat it here.

In the case $a=1$ but $c \neq 0$ the situation is different. We will see that in this case we can solve for $\mu_{n}$ as a function of $\kappa_{n}$ in equation (A.3) for $n$ large. This will enable us to prove that $N$ is a real analytic submanifold of $S$. More precisely our strategy is as follows: given $(\hat{\mu}, \hat{\kappa}) \in N$,

$$
(\hat{\mu}, \hat{\kappa})=\left(\hat{\mu}_{1}, \hat{\kappa}_{1}, \hat{\mu}_{2}, \hat{\kappa}_{2}, \ldots\right)
$$

we need to show that the intersection of $N$ with a neighbourhood of $(\hat{\mu}, \hat{\kappa})$ in $S$ consists of points in $S$ with $\mu_{n}=\mu_{n}\left(\kappa_{n}\right)$ for $n \geq n_{0}$, where each component of the function $\psi(\kappa)=\left(\mu_{n_{0}}\left(\kappa_{n_{0}}\right), \mu_{n_{0}+1}\left(\kappa_{n_{0}+1}\right), \ldots\right)$ is analytic on the ball $\|\kappa-\hat{\kappa}\|<\varepsilon$ in complex-valued $l_{1}^{2}$, and the $l^{2}$-norm of the sequence

$$
\left\{\mu_{n}(\kappa)-n^{2} \pi^{2}-\int_{0}^{1} p d t\right\}_{n=n_{0}}^{\infty}
$$

is uniformly bounded on that ball.

As in $\S 3$, we can then conclude that $\Psi$ is real analytic. Since $N$ for $n \geq n_{0}$ is the graph of $\Psi$ and for $n \leq n_{0}$ we can solve for $\mu_{n}$ or $\kappa_{n}$ locally using real analytic functions, $N$ is a real analytic submanifold of $S$. We will now carry out this plan in detail.

Since $a=1$, we can write $($ A.3) as

$$
\Delta\left(\mu_{n}\right)=2(-1)^{n} \cosh \kappa_{n} .
$$

On $\{\|\kappa-\hat{\kappa}\|<\varepsilon\},\left|\kappa_{n}\right|<2 \varepsilon / n$ for $n>n_{1}(\hat{\kappa}, \varepsilon)$. From this it follows, using Rouché's theorem to compare the roots of $(-1)^{n} \Delta(\lambda)\left(2 \cosh \kappa_{n}\right)^{-1}-1$ and the roots of $(-1)^{n} \cos \sqrt{\lambda}-1$, that there is a constant $A$ and an $n_{2}(\hat{\kappa}, \varepsilon)$ such that for all $\kappa$ in $\{\|\kappa-\hat{\kappa}\|<\varepsilon\},\left(\mathrm{A} .3^{\prime}\right)$ has exactly two roots $\mu_{n}\left(\kappa_{n}\right)$ such that

$$
\sqrt{\mu_{n}}=n \pi+\beta_{n}
$$

with $\left|\beta_{n}\right|<A / n$, when $n \geq n_{2}(\hat{\kappa}, \varepsilon)$. 
Using the asymptotic expansions from (A.1) and analysing the error terms as before, we can write (A.3') in a form analogous to (A.2):

$$
\begin{aligned}
2 \cosh \kappa_{n}= & 2 \cos \beta_{n}+2 \frac{\sin \beta_{n}}{2\left(n \pi+\beta_{n}\right)} \int_{0}^{1} p d t+c \frac{\sin \beta_{n}}{n \pi+\beta_{n}} \\
& -2 \frac{\cos \beta}{8\left(n \pi+\beta_{n}\right)^{2}}\left(\int_{0}^{1} p d t\right)^{2}-c \frac{\cos \beta_{n}}{\left(n \pi+\beta_{n}\right)^{2}} \int_{0}^{1} p d t+r_{n},
\end{aligned}
$$

where the remainders $r_{n}$ are bounded by a fixed sequence in $l_{2}^{2}$ for all sequences $\left\{\beta_{n}\right\}$ satisfying $\left|\beta_{n}\right|<A / n$. Dividing by $2 \cos \beta_{n}$ and denoting another sequence of remainders bounded by a fixed sequence in $l_{2}^{2}$ by $\left\{r_{n}\right\}$,

$$
\frac{\cosh \kappa_{n}}{\cos \beta_{n}}=1+\frac{\beta_{n}}{2 n \pi} \int_{0}^{1} p d t+\frac{c}{2} \frac{\beta_{n}}{n \pi}-\frac{1}{8(n \pi)^{2}}\left(\int_{0}^{1} p d t\right)^{2}-\frac{c}{4(n \pi)^{2}} \int_{0}^{1} q d t+r_{n} .
$$

From the Taylor expansion of $\cos \beta_{n}$ we have

$$
\frac{\cosh \kappa_{n}}{\cos \beta_{n}}=\cosh \kappa_{n}+\frac{\beta_{n}^{2}}{2} \cosh \kappa_{n}+r_{n} \cosh \kappa_{n} .
$$

Substituting this expansion into (A.5) and treating the resulting equation as a quadratic equation for $\beta_{n}$, we obtain

$$
\beta_{n}=\frac{1}{2 \cosh \kappa_{n}}\left(\frac{\int_{0}^{1} p d t+c}{n \pi} \pm \frac{1}{n \pi}\left[c^{2}+8 \cosh \kappa_{n}\left(1-\cosh \kappa_{n}\right) n^{2} \pi^{2}+b_{n}\right]^{1 / 2}\right),
$$

where $\left\{\left|b_{n}\right|\right\}$ is bounded by a fixed $l^{2}$-sequence for all sequences $\left\{\left(\kappa_{n}, \beta_{n}\right)\right\}$ with $\left|\kappa_{n}\right|$ bounded and $\left|\beta_{n}\right|=A / n$. For $\varepsilon$ small enough relative to $c$ it follows that, assuming (A.3') and $\left|\beta_{n}\right| \leq A / n$,

$$
\beta_{n}=\frac{1}{2 n \pi}\left(\int_{0}^{1} p d t+c \pm c+r_{ \pm}(n)\right)
$$

where the error terms $r_{ \pm}(n)$ have $l^{2}$-norm uniformly bounded on $\{\|\kappa-\hat{\kappa}\|<\varepsilon\}$ and there is an $n_{3}(\hat{\kappa}, \varepsilon)$ such that $\left|r_{ \pm}(n)\right|<\varepsilon$ for $n>n_{3}(\hat{\kappa}, \varepsilon)$ uniformly on $\{\|\kappa-\hat{\kappa}\|<\varepsilon\}$. We know that for $n>n_{2}(\hat{\kappa}, \varepsilon),\left(A .3^{\prime}\right)$ does have exactly two solutions $\mu_{n}(k)$ with the corresponding $\beta$ satisfying $\left|\beta_{n}\right|<A / n$. Thus, since we can choose a neighbourhood $O$ of $(\hat{\mu}, \hat{\kappa})$ in $S$ small enough that for any $(\mu, \kappa)$ in $O$ we have $\mid \mu_{n}-n^{2} \pi^{2}-$ $\int_{0}^{1} p d t \mid<\varepsilon$ for $n>n_{4}(\hat{\mu}, \varepsilon)$, and we may assume $\varepsilon<|c|$, we have nearly completed the proof that $N$ is an analytic submanifold. It only remains to show that for $\varepsilon$ sufficiently small and $n$ sufficiently large:

(i) (A.3') could not have two roots satisfying (A.6) with the same choice of sign so that (A.3') does have roots satisfying (A.6) for both choices of sign.

(ii) The roots of $\left(\right.$ A. $\left.3^{\prime}\right)$ in satisfying (A.6) with the minus sign are analytic functions of $\kappa_{n}$ for

$$
\{\|\kappa-\hat{\kappa}\|<\varepsilon\}
$$


Fortunately both (i) and (ii) follow from an estimate on $\partial \Delta / \partial \lambda$ proven by the same method as (A.2) and (A.4):

$$
\frac{\partial \Delta}{\partial \lambda}\left(\mu_{n}\right)=\frac{(-1)^{n}}{2 \pi^{2} n^{2}}\left(2 \pi \beta_{n}-\int_{0}^{1} p d t-c+b_{n}\right),
$$

where $\left|b_{n}\right|$ is bounded by a fixed sequence in $l^{2}$ for $\left|\beta_{n}\right|<A / n$.

In conclusion, let us indicate how we can give $L(p)$ a real analytic structure. For the details of a completely analogous argument see $\S 3$. Using the flows of the vector fields $Z_{n}$, we can show that $\phi(L(p))$ contains all points of $N$ whose coordinates agree with those of $p$ beyond some index $n$, for any finite $n$. Since these sets are dense in $N, L(p)$ is closed in $L_{\mathrm{R}}^{2}[0,1]$ and $\phi$ is a homeomorphism, it follows that $\phi(L(p))=N$. Since $\phi$ is a real analytic homeomorphism, $L(p)$ inherits the real analytic structure of $N$.

\section{Appendix B. Properties of $\Delta(\lambda)$}

In this appendix we give proofs for the properties of $\Delta(\lambda)$ cited in the Introduction. Since these are standard results in Floquet theory, it is quite possible they have appeared elsewhere, but we were unable to locate a reference.

The key facts are:

(i) $\Delta(\lambda)$ is an entire function of order $\frac{1}{2}$ (cf. theorem 1.1).

(ii) All roots of $\Delta(\lambda)=2$ and $\Delta(\lambda)=-2$ are real, since they are eigenvalues of self-adjoint boundary value problems.

(iii) Zeros of $\Delta^{2}(\lambda)-4$ have multiplicity at most 2 .

To prove (iii), it is convenient to use the formula

$$
\Delta^{\prime}(\lambda)=-\int_{0}^{1} \frac{\partial \Delta(\lambda)}{\partial q(x)} d x=-\left[a y_{2}+b y_{2}^{\prime}\right](1, \lambda) \int_{0}^{1} f_{+}(x, \lambda) f_{-}(x, \lambda) d x,
$$

which one obtains by combining theorem 1.2 and formula (2.5). If $\lambda_{0}$ is a zero of $\Delta^{2}(\lambda)-4$, it follows from (B.1) and the definition of $f_{ \pm}$that if $\left[a y_{2}+b y_{2}^{\prime}\right]\left(1, \lambda_{0}\right) \neq 0$, then $f_{+}\left(x, \lambda_{0}\right)=f_{-}\left(x, \lambda_{0}\right)$ and $\Delta^{\prime}\left(\lambda_{0}\right) \neq 0$. If $\lambda_{0}$ is a zero of $\Delta(\lambda)= \pm 2$ of order more than 2 , then both roots of $\xi^{2}-\Delta(\lambda) \xi+1=0$ satisfy

$$
\xi(\lambda)= \pm 1+O\left(\lambda-\lambda_{0}\right)
$$

Thus, since the roots of $\left[a y_{2}+b y_{2}^{\prime}\right](1, \lambda)=0$ must be simple (cf. (1.2)) and $\partial \Delta(\lambda) / \partial q(x) \in L^{2}[0,1]$ for all $\lambda$, it follows that

$$
\lim _{\lambda \rightarrow \lambda_{0}} \int_{0}^{1} f_{+}(x, \lambda) f_{-}(x, \lambda) d x=\int_{0}^{1} g^{2}(x) d x,
$$

where

$$
g(x)=y_{1}\left(x, \lambda_{0}\right)+\left(\lim _{\lambda \rightarrow \lambda_{0}}\left[\frac{\xi(\lambda)-a y_{1}-b y_{1}^{\prime}}{a y_{2}+b y_{2}^{\prime}}\right](1, \lambda)\right) y_{2}\left(x, \lambda_{0}\right) .
$$

However, by (B.1) this implies $\Delta^{\prime}(\lambda)$ has a simple zero at $\lambda=\lambda_{0}$. Thus we conclude (iii) holds. 
Combining (i) and (ii), Hadamard's theorem gives the product representations

$$
\Delta(\lambda) \pm 2=a_{ \pm} \lambda^{k_{ \pm}} \prod_{j=1}^{\infty}\left(1-\frac{\lambda}{a_{j}^{ \pm}}\right),
$$

where the $a_{j}^{ \pm}$are real and increasing with $j$. Hence

$$
\begin{gathered}
\frac{\Delta^{\prime}(\lambda)}{\Delta(\lambda) \pm 2}=\frac{k_{ \pm}}{\lambda}+\sum_{j=1}^{\infty} \frac{1}{\lambda-a_{j}^{ \pm}} \\
\left(\frac{\Delta^{\prime}(\lambda)}{\Delta(\lambda) \pm 2}\right)^{\prime}=\frac{-k_{ \pm}}{\lambda^{2}}-\sum_{j=1}^{\infty} \frac{1}{\left(\lambda-a_{j}\right)^{2}}<0 .
\end{gathered}
$$

Thus, by (B.3), $\Delta^{\prime}(\lambda)(\Delta(\lambda) \pm 2)^{-1}$ is strictly decreasing in $\lambda$. Then, letting $\left\{\delta_{j}\right\}_{j=1}^{\infty}$ denote the real zeros of $\Delta^{\prime}(\lambda)$ and recalling that $\Delta(\lambda) \rightarrow+\infty$ as $\lambda \rightarrow-\infty$ (cf. theorem 1.1), we see from (B.2) that

$$
\lambda_{0}<\lambda_{1} \leq \delta_{1} \leq \lambda_{2}<\lambda_{3} \leq \delta_{2} \leq \lambda_{4} \cdots .
$$

Moreover, $\lambda_{2 j-1}<\delta_{j}<\lambda_{2 j}$ unless $\lambda_{2 j-1}=\lambda_{2 j}$. This establishes the result on the critical values of $\Delta(\lambda)$ cited in the Introduction.

To show that the multiplicity of the $\lambda_{j}$ as roots of $\Delta^{2}(\lambda)-4$ equals their multiplicity as eigenvalues, we note that if $\lambda_{0}$ is a zero of order 2 , then $\left[a y_{2}+b y_{2}^{\prime}\right](1, \lambda)=0$ by the analysis in the preceding paragraph, and $\left[a y_{1}+b y_{1}^{\prime}\right](1, \lambda)= \pm 1$, since $B F\left(\lambda_{0}\right)$ must have eigenvalues equal to \pm 1 . The identity (cf. lemma 4.2(iv))

$$
\left[y_{2}^{2}+b \Delta(\lambda) y_{2}+b^{2}\right](1, \lambda)=\left[\left(a y_{2}+b y_{2}^{\prime}\right)\left(d y_{2}+b_{1}\right)\right](1, \lambda)
$$

then implies $y_{2}\left(1, \lambda_{0}\right)=\mp b$, which in turn implies that the right-hand side of (B.4) vanishes to second order at $\lambda=\lambda_{0}$ and hence $\left[d y_{2}+b y_{1}\right]\left(1, \lambda_{0}\right)=0$. Thus one has $y_{2}\left(1, \lambda_{0}\right)=\mp b, y_{2}^{\prime}\left(1, \lambda_{0}\right)= \pm a, y_{1}\left(1, \lambda_{0}\right)= \pm d$ and $y_{2}^{\prime}\left(1, \lambda_{0}\right)=\mp c$, which implies $B F\left(\lambda_{0}\right)= \pm I$ and $\lambda_{0}$ is an eigenvalue of multiplicity 2 .

Conversely, if $\lambda_{0}$ is a simple zero of $\Delta^{2}(\lambda)-4$, then either $\left[a y_{2}+b y_{2}^{\prime}\right]\left(1, \lambda_{0}\right) \neq 0$, which implies $B F\left(\lambda_{0}\right) \neq \pm I$, or, if $\left[a y_{2}+b y_{2}^{\prime}\right]\left(1, \lambda_{0}\right)=0$, then (B.4) implies $y_{2}\left(1, \lambda_{0}\right)=$ $\mp b$ and $\left[d y_{2}+b y_{1}\right]\left(1, \lambda_{0}\right) \neq 0$. Hence $y_{1}\left(1, \lambda_{0}\right) \neq \pm d$. However, this shows

$$
\left[c y_{1}+d y_{1}^{\prime}\right]\left(1, \lambda_{0}\right)=\left[\frac{-y_{1}}{b} \pm \frac{d}{b}\right]\left(1, \lambda_{0}\right)
$$

is non-zero, and we again conclude $B F\left(\lambda_{0}\right) \neq \pm I$. Thus $\lambda_{0}$ is a simple eigenvalue.

\section{REFERENCES}

[1] B. E. J. Dahlberg \& E. Trubowitz. The inverse Sturm-Liouville problem III. Commun. Pure Appl. Math. 37 (1984), 255-268.

[2] J. Garnett \& E. Trubowitz. Gaps and bands of one-dimensional periodic Schrödinger operators. Commun. Math. Helv. 59 (1984), 258-312.

[3] E. Isaacson, H. McKean \& E. Trubowitz. The inverse Sturm-Liouville problem II. Commun. Pure Appl. Math. 37 (1984), 1-12.

[4] E. Isaacson \& E. Trubowitz. The inverse Sturm-Liouville problem I. Commun. Pure Appl. Math. 36 (1983), 767-783.

[5] E. Trubowitz. The inverse problem for periodic potentials. Commun. Pure Appl. Math. 30 (1977), 321-337.

[6] J. Pöschel \& E. Trubowitz. Inverse Spectral Theory. Academic Press (1987). 